\title{
A Hamiltonian analogue of the meandering transition
}

\author{
Claudia Wulff \\ Department of Mathematics, University of Surrey \\ Guildford GU2 7XH, UK \\ c.wulff@surrey.ac.uk
}

June 5, 2008

\begin{abstract}
In this paper a Hamiltonian analogue of the well-known meandering transition from rotating waves to modulated rotating and modulated travelling waves in systems with the Euclidean symmetry of the plane is presented. In non-Hamiltonian systems, for example in spiral wave dynamics, this transition is a Hopf bifurcation in a corotating frame, as external parameters are varied, and modulated traveling waves only occur at certain resonances. In Hamiltonian systems, for example in systems of point vortices in the plane, the conserved quantities of the system, angular and linear momentum, are natural bifurcation parameters. Depending on the symmetry properties of the momentum map, either modulated traveling waves do not occur, or, in contrast to the dissipative case, modulated traveling waves are the typical scenario near rotating waves, as momentum is varied. Systems with the symmetry group of a sphere and with the Euclidean symmetry group of three space are also treated.
\end{abstract}

AMS subject classification. 37J15, 37J20, 53D20, 70H33

Keywords. Meandering transition, resonance drift, symmetric Hamiltonian systems.

\section{Contents}

1 Introduction $r$

2 Meandering transition for dissipative systems $\quad 3$

2.1 Relative equilibria and relative periodic orbits of general systems . . . . . . . . 3

2.2 The meandering transition for dissipative systems . . . . . . . . . . . . 5

3 Dynamics near Hamiltonian relative equilibria $\quad 8$

3.1 Symmetric Hamiltonian systems . . . . . . . . . . . . . . . . . . . 8

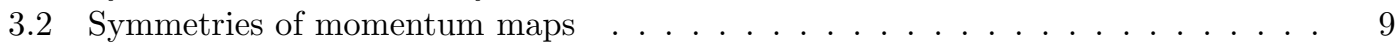

3.3 Dynamics near Hamiltonian relative equilibria . . . . . . . . . . . . . . . 11

4 The meandering transition in Hamiltonian systems $\quad 14$

4.1 Persistence of rotating waves . . . . . . . . . . . . . . . . . . . . . . . 14

4.2 Bifurcation of modulated travelling waves . . . . . . . . . . . . . . . . . . . . . . . . . . . . . . . . . . . .

4.3 Bifurcation of modulated rotating waves . . . . . . . . . . . . . . . . 15

4.4 Hamiltonian meandering transition for momentum maps with cocycle . . . . . . 18

5 Extensions to systems with other symmetry groups 22

5.1 Hamiltonian meandering transition with spherical symmetry . . . . . . . . . . . . 22

5.2 Hamiltonian meandering transition with the Euclidean symmetry of threedimensional space . . . . . . . . . . . . . . . . . 25

$\begin{array}{lr}\text { Conclusion and future directions } & 29\end{array}$ 


\section{Introduction}

The meandering transition in spiral wave dynamics is a transition from rigidly rotating to meandering and drifting spiral waves. In symmetry terms, it is a bifurcation from rotating waves to modulated rotating and modulated traveling waves in systems with $\mathrm{SE}(2)$-symmetry. Here $\mathrm{SE}(2)$ is the special Euclidean group of motions of the plane. Rotating waves are solutions which become stationary in a corotating frame and are examples of relative equilibria. Modulated rotating and modulated traveling waves are solutions which become periodic in a corotating/comoving frame and are examples of relative periodic orbits (RPOs). In non-Hamiltonian systems, the meandering bifurcation corresponds, in a rotating frame, to a Hopf bifurcation induced by changing an external parameter. Typically the bifurcating relative periodic orbits are modulated rotating waves, and modulated traveling waves only occur at certain resonances. See for example $[3,8,10,27,30,31]$ and the references therein.

In this paper the first ever analysis of the Hamiltonian analogue of this meandering transition is presented. Examples of Hamiltonian systems where such a transition occurs are rotating point vortices on the plane $[1,2,21,25,29]$ or rotating rigid bodies in ideal fluids [15]. In a Hamiltonian system it is natural to study the persistence and bifurcation of the rotating wave to nearby momentum levels since the momentum map is a conserved quantity and hence an internal parameter of the system.

The differential equations near Hamiltonian relative equilibria in symmetry-adapted local coordinates from [26] are used to study the transition from rotating waves to modulated rotating and modulated traveling waves on nearby momentum levels in Hamiltonian systems with SE(2)symmetry. Thereby a Hamiltonian analogue of the meandering transition of spiral waves is obtained.

It is shown that, depending on the symmetry properties of the momentum map, either modulated traveling waves are typical near rotating waves, as momentum is varied (cf. Sections $4.2,4.3$ ), or that modulated traveling waves do not occur, see Section 4.4 and in particular Proposition 4.10. As far as I am aware, for the first time, rotating waves and transitions to relative periodic orbits are continued in the cocycle parameter which determines the symmetry properties of the momentum map. These results hold under conditions which are generically satisfied.

The transition from rotating waves to modulated traveling waves occuring in the meandering transition is an example of resonance drift, as analyzed in [31], see also [4] and [6]. Resonance drift occurs if there is a discontinuity of the average drift velocities of the bifurcating relative periodic orbits at the relative equilibrium. In the case of the meandering transition it is a discontinuous jump between a rotational and a translational velocity. This phenomenon is also discussed in systems with spherical symmetry $\mathrm{SO}(3)$ and in systems with the Euclidean symmetry $\mathrm{SE}(3)$ of motions in three-dimensional space, see Sections 5.1, 5.2.

The meandering transition is a transition from relative equilibria to relative periodic orbits. In non-Hamiltonian systems it is a Hopf bifurcation of the symmetry reduced dynamics. The Hamiltonian analogue of a Hopf bifurcation is a Lyapounov centre bifurcation. In this paper Lyapounov centre bifurcations for the reduced Hamiltonian system on the symplectic slice are proved to obtain families of RPOs nearby elliptic relative equilibria, see Propositions 4.6, Theorems $4.11 \mathrm{a}$ ), $5.1 \mathrm{~b}), 5.2 \mathrm{c}$ ), Propositions $5.3 \mathrm{~b}$ ) and 5.6.

The technically most complicated parts of the paper are the results on bifurcation from relative equilibria to RPOs which lie outside the symplectic leaf of the original equilibrium of the reduced dynamics, see Theorems 4.3, 5.2 b), 5.5. Here Lyapounov centre type theorems are proved for the symmetry reduced system which is a Poisson system and not a Hamiltonian system. It is shown that in this case resonance drift occurs.

Related results in the literature are the following: Persistence results for generic Hamiltonian relative equilibria and relative periodic orbits of noncompact group actions, extending earlier results for compact symmetry groups, can be found in [32, 33]. See also Ortega and Ratiu [23] and Montaldi and Tokieda [20] and references therein for results on bifurcations of Hamiltonian relative equilibria.

Relative Lyapounov centre bifurcations from Hamiltonian relative equilibria with isotropy to 
RPOs, which lie on nearby energy-level sets, have been obtained by Ginzburg and Lerman [9] (see also references therein). Ortega [22] studies persistence of the bifurcating RPOs to nearby energy level sets and to those nearby momentum values which correspond to the isotropy subgroup of the relative equilibrium. Instead, in this article, the group is assumed to act freely, and the main focus is the bifurcation of relative equilibria to RPOs on all nearby momentum level sets.

The paper is organized as follows: In Section 2 the meandering transition for dissipative systems is reviewed. In Section 3 symmetric Hamiltonian systems are introduced and the equations near relative equiibria from [26] are reviewed. In Section 4 a Hamiltonian analogue of the meandering transition is presented using the equations near Hamiltonian relative equilibria from Section 3. First Euclidean symmetric Hamiltonian systems with an equivariant momentum map for the standard coadjoint action are studied. Then systems with Euclidean symmetry for which the momentum map has a cocycle are considered. Finally, in Section 5, the Hamiltonian analogue of the meandering transition is discussed for systems with spherical symmetry and for systems with the Euclidean symmetry group of three-dimensional space.

\section{Meandering transition for dissipative systems}

In this section the notions of relative equilibria and relative periodic orbits of general symmetric differential equations are defined. Suitable symmetry-adapted coordinates near relative equilibria are introduced, and the differential equations are given in these coordinates. Then the results are applied to dissipative systems with the Euclidean symmetry of the plane, and the meandering transition for dissipative systems is reviewed. Note that in this paper the terms "dissipative system", "non-Hamiltonian systems" and "general systems" are used interchangably. Most of the material of this section is basically contained in $[8,10,31]$. Only Remark $2.2 \mathrm{c}$ ) is a new result.

\subsection{Relative equilibria and relative periodic orbits of general systems}

Let us consider an ordinary differential equation on a finite-dimensional manifold $\mathcal{M}$

$$
\dot{x}(t)=f(x(t))
$$

with flow $\Phi_{t}\left(x_{0}\right)=x\left(t ; x_{0}\right), x(0)=x_{0}$. Let a finite dimensional Lie group $\Gamma$ act properly and smoothly on $\mathcal{M}$. For simplicity it is assumed that the $\Gamma$-action is free, that is,

$$
\Gamma_{x}=\{\gamma \in \Gamma, \quad \gamma x=x\}=\{\mathrm{id}\}
$$

for all $x \in \mathcal{M}$. The vectorfield (2.1) is taken to be $\Gamma$-equivariant, i.e.,

$$
\gamma f(x)=f(\gamma x) \text { for all } \gamma \in \Gamma .
$$

A solution $x(t)$ with initial condition $x(0)=x_{0}$ lies on a relative equilibrium $\Gamma x_{0}$ whenever the group orbit $\Gamma x_{0}$ is invariant under the flow of (2.1), i.e., if $x\left(t ; x_{0}\right) \in \Gamma x_{0}$ for all $t$. This means that

$$
f\left(x_{0}\right)=\xi_{0} x_{0}:=\left.\left(\frac{\mathrm{d}}{\mathrm{d} s} \exp \left(s \xi_{0}\right) \cdot x_{0}\right)\right|_{s=0}
$$

for some $\xi_{0} \in \mathbf{g}$. Here $\mathbf{g}=\mathcal{T}_{\text {id }} \Gamma$ is the Lie algebra of $\Gamma$. The element $\xi_{0}$ is called the drift velocity of the relative equilibrium at $x_{0}$. Note that the trajectory through $x_{0}$ becomes an equilibium in a frame moving with velocity $\xi_{0}$. If $\xi_{0}$ is an infinitesimal rotation then the relative equilibrium is called a rotating wave (RW). Note that at the point $\gamma x_{0}$ of the relative equilibrium $\Gamma x_{0}$ the drift velocity is determined by the equation

$$
f\left(\gamma x_{0}\right)=\gamma f\left(x_{0}\right)=\gamma \xi_{0} x_{0}=\left(\operatorname{Ad}_{\gamma} \xi_{0}\right) x_{0}
$$

and is therefore given by $\operatorname{Ad}_{\gamma} \xi_{0}$. Here $\operatorname{Ad}_{\gamma}: \mathbf{g} \rightarrow \mathbf{g}$ and

$$
\operatorname{Ad}_{\gamma} \eta=\gamma \eta \gamma^{-1}, \quad \operatorname{ad}_{\xi} \eta=\left.\frac{\mathrm{d}}{\mathrm{d} t} \operatorname{Ad}_{\exp (t \xi)} \eta\right|_{t=0}=[\xi, \eta], \quad \gamma \in \Gamma, \eta, \xi \in \mathbf{g},
$$


are the adjoint action of $\Gamma$ and $\mathbf{g}$ and the infinitesimal adjoint action of $\mathbf{g}$ on $\mathbf{g}$.

An example of such a finite-dimensional manifold $\mathcal{M}$ with SE(2)-equivariant vectorfield (2.1) on it is the centre manifold near a rotating spiral $\mathrm{SE}(2) x_{0}$ in a reaction-diffusion system, see e.g. [27]. Here $\mathrm{SE}(2)=\mathrm{SO}(2) \ltimes \mathbb{R}^{2}$ is the special Euclidean symmetry of rotations and translations in the plane with group multiplication defined in (2.5) below.

By the slice theorem of Palais [24] sufficiently small neighbourhoods $\mathcal{U}$ of the group orbit $\Gamma x_{0}$ have the bundle structure $\mathcal{U}=\Gamma \times \mathcal{N}$. Here $\mathcal{N} \subseteq \mathcal{T}_{x_{0}} \mathcal{M}$ is a local section, also called slice, transversal to $\Gamma x_{0}$ at $x_{0}$, see Figure 1.

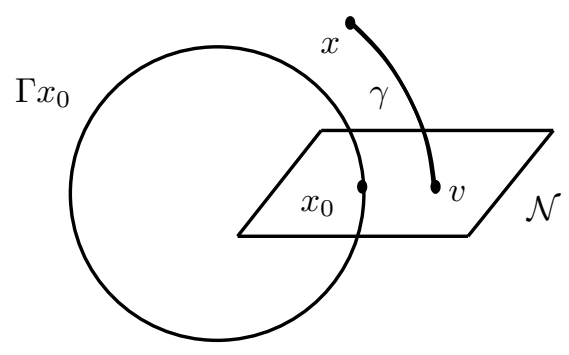

Figure 1: Palais coordinates near $\Gamma x_{0}$

To analyze the dynamics near, and bifurcations from, relative equilibria, it has proved very useful to model the flow in a $\Gamma$-invariant neighbourhood $\mathcal{U}$ of the relative equilibrium by differential equations on the space $\Gamma \times \mathcal{N}$ :

$$
\dot{\gamma}=\gamma f_{\Gamma}(v), \quad \dot{v}=f_{\mathcal{N}}(v),
$$

where $f_{\Gamma}: \mathcal{N} \rightarrow \mathbf{g}$ and $f_{\mathcal{N}}: \mathcal{N} \rightarrow \mathcal{N}$. Any $x \in \mathcal{U}$ takes the form $x \simeq(\gamma, v) \in \Gamma \times \mathcal{N}$, and the point $x_{0}$ corresponds to $x_{0} \simeq(\mathrm{id}, 0)$. Then $f_{\mathcal{N}}(0)=0$, i.e., the relative equilibrium $\Gamma x_{0}$ of $(2.1)$ becomes an equilibrium of the $\dot{v}$-equation. Moreover $f_{\Gamma}(0)=\xi_{0}$. Note that the equations (2.3) have skew-product form: the $\dot{v}$-equation, which is called the slice equation, does not depend on the group variable $\gamma$. It describes the symmetry-reduced dynamics, whereas the $\dot{\gamma}$-equation describes the drift dynamics on the group $\Gamma$. These results are due to Krupa [14] for compact Lie groups and due to Fiedler et al. [8] for noncompact Lie groups. For later use, the linearization $L_{0}=\mathrm{D} f\left(x_{0}\right)-\xi_{0}$ of the relative equilibrium $\Gamma x_{0}$ at $x_{0}$ in the frame moving with the velocity $\xi_{0}$ in symmetry adapted coordinates is

$$
L_{0}=\left(\begin{array}{ll}
\operatorname{ad}_{\xi_{0}} & \mathrm{D}_{v} f_{\Gamma}(0) \\
0 & \mathrm{D}_{v} f_{\mathcal{N}}(0)
\end{array}\right)
$$

The point $x_{0} \in \mathcal{M}$ lies on a relative periodic orbit (RPO) $\mathcal{P}$ of $(2.1)$ if $x\left(t ; x_{0}\right)=\Phi_{t}\left(x_{0}\right)$ is periodic in the space of group orbits $\mathcal{M} / \Gamma$. This means that there exists $T_{0}>0$ and $\gamma_{0} \in \Gamma$

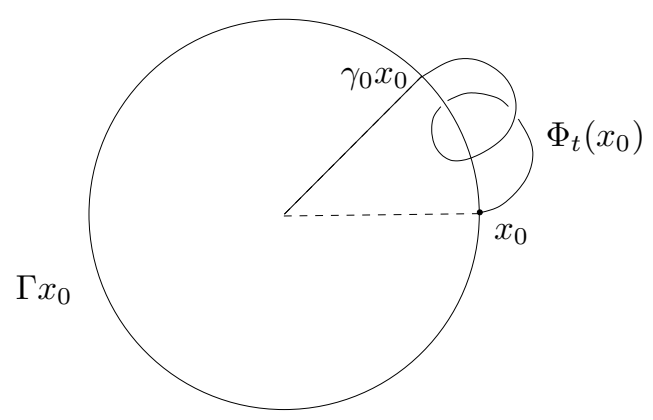

Figure 2: A relative periodic orbit 
such that $\Phi_{T_{0}}\left(x_{0}\right)=\gamma_{0} x_{0}$, see Figure 2 . The infimum of the numbers $T_{0}$ with this property is the relative period of the relative periodic orbit. The corresponding group element $\gamma_{0}$ is called the drift symmetry of the relative periodic orbit with respect to $x_{0}$, c.f. $[33,35]$. The relative periodic orbit itself is defined to be the submanifold of $\mathcal{M}$ given by

$$
\mathcal{P}=\left\{\gamma \Phi_{t}\left(x_{0}\right) \mid \gamma \in \Gamma, t \in \mathbb{R}\right\} .
$$

If $\gamma_{0}$ is a translation the relative periodic orbit is called a modulated traveling wave (MTW); if $\gamma_{0}$ is a non-vanishing translation it is a proper modulated traveling wave. If $\gamma_{0}$ is a (non-vanishing) rotation the RPO is called a (proper) modulated rotating wave (MRW), see Figure 3 . Any $\xi_{0} \in \mathbf{g}$ such that $\gamma_{0}=\exp \left(T_{0} \xi_{0}\right)$ is called an average drift velocity of the RPO at $x_{0}$. Note that the trajectory through $x_{0}$ becomes $T_{0}$-periodic in a frame moving with velocity $\xi_{0}$.

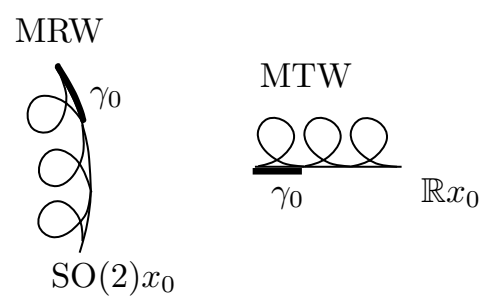

Figure 3: Drift symmetries of modulated rotating and modulated travelling waves

\subsection{The meandering transition for dissipative systems}

Let $\Gamma$ be the Euclidean symmetry of the plane consisting of rotations and translations

$$
\Gamma=\mathrm{SE}(2)=\mathrm{SO}(2) \ltimes \mathbb{R}^{2},
$$

where the semidirect product is defined as

$$
\left(\phi_{1}, a_{1}\right)\left(\phi_{2}, a_{2}\right)=\left(\phi_{1}+\phi_{2}, a_{1}+R_{\phi_{1}} a_{2}\right), \quad \phi_{i} \in \mathrm{SO}(2), a_{i} \in \mathbb{R}^{2}, i=1,2 .
$$

Here $R_{\phi}$ is a rotation by $\phi$ in $\mathbb{R}^{2}$. Let us assume that the relative equilibrium $\operatorname{SE}(2) x_{0}$ is a rotating wave with rotation frequency $\omega_{0}^{\text {rot }}$. Then the $\dot{\gamma}$-equation in $(2.3)$, which models the drift dynamics near the rotating wave, takes the following form:

$$
\dot{\phi}=f_{\phi}(v), \quad \dot{a}=R_{\phi} f_{a}(v) .
$$

Moreover $f_{\phi}(0)=\omega_{0}^{\text {rot }}$ is the rotation frequency of the rotating wave and $f_{a}(0)=0$. As in the general case, the rotating wave $\operatorname{SE}(2) x_{0}$ becomes an equilibrium of the slice equation: $f_{\mathcal{N}}(0)=0$. These equations have first been formulated by Barkley [3] and have then been derived by Fiedler et al [8] and Golubitsky et al [10].

Let us now assume that both $f_{\mathcal{N}}(\cdot, \mu)$ and $f_{\Gamma}(\cdot, \mu)=\left(f_{\phi}(\cdot, \mu), f_{a}(\cdot, \mu)\right)$ depend on an external parameter $\mu \in \mathbb{R}$. In a meandering transition the symmetry reduced system undergoes a Hopf bifurcation. Suppose that this bifurcation occurs for $\mu=0$, let $\pm \mathrm{i} \omega_{0}^{\text {Hopf }}$ be the Hopf eigenvalues of $\mathrm{D}_{v} f_{\mathcal{N}}(0,0)$. Assume that $\pm \mathrm{i} \omega_{0}^{\text {Hopf }}$ are simple eigenvalues and that $\mathrm{D} f_{\mathcal{N}}(0,0)$ has no other eigenvalues in $\mathrm{i} \omega_{0}^{\mathrm{Hopf}} \mathbb{Z}$. Let $v_{\mathrm{RW}}(\mu) \approx 0$ be the equilibrium of $f_{\mathcal{N}}(\cdot, \mu), \mu \approx 0$, such that $v_{\mathrm{RW}}(\mu)$ is smooth in $\mu$ and $v_{\mathrm{RW}}(0)=0$. Then $x_{\mathrm{RW}}(\mu) \simeq\left(\mathrm{id}, v_{\mathrm{RW}}(\mu)\right)$ lies on a rotating wave of (2.1). Let $\lambda(\mu)$ be the eigenvalue of $\mathrm{D}_{v} f_{\mathcal{N}}\left(v_{\mathrm{RW}}(\mu), \mu\right)$ such that $\lambda(\mu)$ is smooth in $\mu$ and $\lambda(0)=\mathrm{i} \omega_{0}^{\text {Hopf }}$. Assume that the usual transversality condition

$$
\left.\operatorname{Re} \frac{\partial}{\partial \mu} \lambda(\mu)\right|_{\mu=0} \neq 0
$$

for Hopf bifurcation is satisfied. Then there is a smooth path $v(s), s \geq 0$, of points on periodic solutions of the $\dot{v}$-equation with period $T(s) \approx T_{0}^{\mathrm{Hopf}}=2 \pi / \omega_{0}^{\mathrm{Hopf}}$ and parameter $\mu(s)$ such that $v(0)=0, T(0)=T_{0}^{\mathrm{Hopf}}, \mu(0)=0$. 
The periodic orbit through $v(s)$ of the slice equation corresponds to a relative periodic orbit $\mathcal{P}(s)$ through $x(s) \simeq(\mathrm{id}, v(s))$ of the original ODE (2.1) with drift symmetry $\gamma(s)=(\phi(s), a(s))$. Here $\phi(s)$ and $a(s)$ are obtained by integrating $(2.6)$ from 0 to $T(s)$. There are two cases:

a) If $\phi(s) \neq 0$ mod $2 \pi$ then $x(s)$ lies on a modulated rotating wave, and this is the typical case;

b) If $\phi(s)=0 \bmod 2 \pi$ then $x(s)$ lies on a modulated travelling wave.

Note that

$$
\phi(s) \approx \omega_{0}^{\mathrm{rot}} T_{0}^{\mathrm{Hopf}}=\frac{\omega_{0}^{\mathrm{rot}}}{\omega_{0}^{\mathrm{Hopf}}} 2 \pi .
$$

Hence case b) occurs if $\frac{\omega_{0}^{\text {rot }}}{\omega_{0}^{\text {Hopf }}} \in \mathbb{Z}$, i.e., if there is a resonance between the rotation frequency $\omega_{0}^{\text {rot }}$ and the Hopf frequency $\omega_{0}^{\text {Hopf }}$ of the rotating wave $\Gamma x_{0}$, see $[3,8,10,31]$. In the case of two real parameters $\mu \in \mathbb{R}^{2}$ the following proposition holds true, see also Figure 4 :

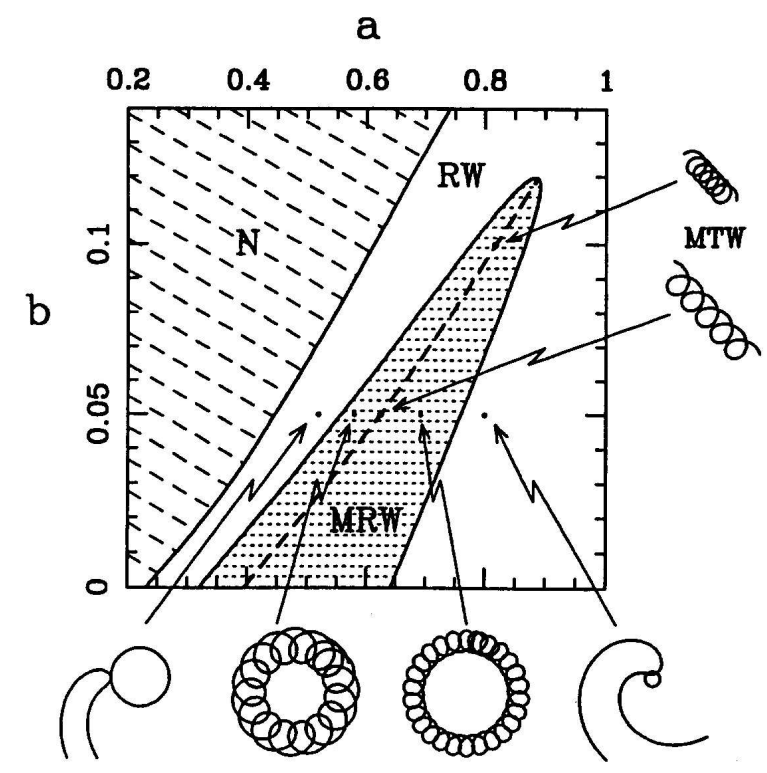

Figure 4: Phase diagram for the spiral wave dynamics for a reaction-diffusion system depending on the parameters $a, b$. Reprinted Fig. 1 with permission from [3], copyright 1994 by the American Physical Society ${ }^{1}$. Shown are regions containing N: no spiral waves, RW: stable rigidly rotating waves, MRW: modulated rotating waves, MTW: modulated travelling waves (dashed curve). Spiral tip paths illustrate states at 6 points. Small portions of spiral waves are shown for the two rotating wave cases.

Proposition 2.1 [31, Example 3.6] Let $\mu \in \mathbb{R}^{2}$, let $\mathrm{SE}(2) x_{0}$ be a rotating wave at $\mu=0$ at which a resonant Hopf bifurcation occurs: $\frac{\omega_{0}^{\text {rot }}}{\omega_{0}^{\text {Hopf }}} \in \mathbb{Z}$. Then under some nondegeneracy conditions (detailed in the proof below) a path $\mathcal{P}_{\mathrm{MTW}}(s), s \geq 0$, of modulated travelling waves at parameters $\mu_{\mathrm{MTW}}(s)$ bifurcates from the rotating wave $\mathrm{SE}(2) x_{0}$.

Proof. Denote the rotation frequency of the rotating wave $\mathrm{SE}(2) x_{\mathrm{RW}}(\mu)$ at parameter $\mu$ by $\omega^{\text {rot }}(\mu)$. The transversality condition (2.7) for Hopf bifurcation insures that coordinates in

\footnotetext{
${ }^{1}$ Readers may view, browse and/or download material for temporary copying purposes only, provided these uses are for noncommercial personal purposes. Except as provided by law, this material may not be further reproduced, distributed, transmitted, modified, adapted, performed, displayed, published, or sold in whole or part, without prior written permission from the American Physical Society.
} 
parameter space $\mu \in \mathbb{R}^{2}$ can be chosen such that $\mu_{1}=0$ is the Hopf line near $\mu=0$, i.e., such that $\lambda\left(0, \mu_{2}\right)=\mathrm{i} \omega^{\operatorname{Hopf}}\left(\mu_{2}\right)$ for some smooth function $\omega^{\operatorname{Hopf}}\left(\mu_{2}\right)$ with $\omega^{\text {Hopf }}(0)=\omega_{0}^{\text {Hopf }}$. Periodic orbits bifurcating from this Hopf line are then parametrized by $\mu_{2}$ and $s \geq 0$. Let $v\left(s, \mu_{2}\right)$ lie on a periodic orbit with parameters $s, \mu_{2}$ such that $v\left(s, \mu_{2}\right)$ is smooth in its parameters and $v\left(0, \mu_{2}\right)=v_{\mathrm{RW}}\left(0, \mu_{2}\right)$. Let $T\left(s, \mu_{2}\right)$ be the period of the periodic orbit through $v\left(s, \mu_{2}\right)$. Modulated travelling waves satisfy $F\left(s, \mu_{2}\right)=\phi\left(T\left(s, \mu_{2}\right)\right)=0$. This equation can be solved near 0 for $\mu_{2}(s)$ by the implicit function theorem if $\frac{\partial F}{\partial \mu_{2}}(0) \neq 0$. This condition holds true if the nondegeneracy condition

$$
\left.\frac{\partial}{\partial \mu_{2}}\left(\frac{\omega^{\operatorname{rot}}\left(\mu_{2}\right)}{\omega^{\operatorname{Hopf}}\left(\mu_{2}\right)}\right)\right|_{\mu_{2}=0} \neq 0
$$

is satisfied. Then $v\left(s, \mu_{2}(s)\right)$ lies on modulated travelling wave $\mathcal{P}_{\mathrm{MTW}}(s)$.

\section{Remarks 2.2}

a) In [31] (see also [6] for compact groups) resonances of the form $\omega_{\Gamma} / \omega_{\mathcal{N}}=k \in \mathbb{Z} \backslash\{0\}$ between a non-vanishing imaginary eigenvalue $\pm \mathrm{i} \omega_{\Gamma}$ of $\operatorname{ad}_{\xi_{0}}$ and an eigenvalue $\pm \mathrm{i} \omega_{\mathcal{N}}$ of $\mathrm{D}_{v} f_{\mathcal{N}}(0)$ are shown to be necessary for resonance drift to occur. Resonance drift means that $\operatorname{RPOs} \mathcal{P}(s)$ bifurcate with average drift velocities $\xi(s)$ at $x(s) \in \mathcal{P}(s)$ which cannot be chosen to converge to the drift velocity $\xi_{0}$ of the relative equilibrium, i.e., $x(s) \rightarrow x_{0}$ as $s \rightarrow 0$, but $\lim _{s \rightarrow 0} \xi(s) \neq \xi_{0}$. From the form of the linearization $L_{0}=\operatorname{D} f\left(x_{0}\right)-\xi_{0}$ about a relative equilibrium $\Gamma x_{0}$ in a corotating frame, see $(2.4)$, it follows that resonance drift is caused by resonances between drift dynamics and the symmetry-reduced dynamics. In Proposition 2.1 above, resonance drift occurs with $\omega_{\Gamma}=\omega_{0}^{\text {rot }}$ and $\omega_{\mathcal{N}}=\omega_{0}^{\text {Hopf }}$. Proposition 2.1 is a special case of [31, Proposition 3.4], which treats resonance drift for general Lie groups $\Gamma$.

b) In the case of spherical symmetry $\Gamma=\mathrm{SO}(3)$, modeling for example rotating spiral waves of reaction-diffusion systems on the sphere, resonance drift caused by resonant Hopf bifurcation has been studied in $[31,7,4]$. In this case generically there is a path $(x(s), \mu(s))$ in two dimensional parameter space $\mu(s) \in \mathbb{R}^{2}$ such that $\mathrm{SO}(3) x(s)$ is a modulated rotating wave at parameter $\mu(s)$ with an average drift velocity $\xi(s)$ at $x(s)$ which is orthogonal to the drift velocity $\xi_{0} \in \mathrm{So}(3) \in \mathcal{T}_{\text {id }} \mathrm{SO}(3)$ of the rotating wave $\mathrm{SO}(3) x_{0}$ at $x_{0}=\lim _{s \rightarrow 0} x(s)$. The proof of this result is very similar to the proof of Proposition 2.1: For any $R \in \mathrm{SO}(3)$ write

$$
R=\exp \left(\sum_{i=1}^{3} \phi_{i} \xi_{i}\right) .
$$

Here $\xi_{i}, i=1,2,3$, are infinitesimal rotations such that $\exp \left(\phi_{i} \xi_{i}\right), i=1,2,3$, is a rotation by the angle $\phi_{i}$ around the $e_{i}$ axis (often so(3) is identified with $\mathbb{R}^{3}$ and $\xi_{i}$ with $e_{i}, i=1,2,3$ ). Assume, as before, that the Hopf line is at $\mu_{1}=0$. Let $R\left(s, \mu_{2}\right)$ be the drift symmetry of the modulated rotating wave at $x\left(s, \mu_{2}\right)$. Assume, without loss of generality, that the rotating wave through $x_{\mathrm{RW}}(\mu)$ has a rotation velocity $\xi_{\mathrm{RW}}(\mu) \| \xi_{3}$ so that $\xi_{\mathrm{RW}}(\mu)=\omega^{\mathrm{rot}}(\mu) e_{3}$. Then the modulated rotating waves to be found satisfy the equation $F\left(s, \mu_{2}\right)=\phi_{3}\left(s, \mu_{2}\right)=0$. This equation can be solved if (2.8) holds. The bifurcating modulated rotating waves $\mathcal{P}(s)$ have average drift velocities in the $\left(x_{1}, x_{2}\right)$-plane. For a Hamiltonian analogue see Section 5.1 .

c) Resonance drift also occurs for relative equilibria of systems with the Euclidean symmetry group $\Gamma=\mathrm{SE}(3)=\mathrm{SO}(3) \ltimes \mathbb{R}^{3}$ of rotations and translations in three-dimensional space, c.f. [5]. An example would be a Hopf bifurcation from a rigidly rotating and translating scroll wave $\mathrm{SE}(3) x_{0}$ of a reaction-diffusion system on $\mathbb{R}^{3}$, see e.g. [30]. The group multiplication on $\Gamma=\mathrm{SE}(3)=\mathrm{SO}(3) \ltimes \mathbb{R}^{3}$ is analogous to $(2.5)$ : For $\left(R_{1}, a_{1}\right),\left(R_{2}, a_{2}\right) \in \mathrm{SO}(3) \ltimes \mathbb{R}^{3}$ it is given by

$$
\left(R_{1}, a_{1}\right)\left(R_{2}, a_{2}\right)=\left(R_{1} R_{2}, a_{1}+R_{1} a_{2}\right), \quad R_{1}, R_{2} \in \mathrm{SO}(3), \quad a_{1}, a_{2} \in \mathbb{R}^{3} .
$$


Note that $(R, a)$ is a rotation around the rotation axis of $R$ about the point

$$
c=(\mathrm{id}-R)^{+} a \in \mathbb{R}^{3}
$$

combined with a translation along the axis of $R$. Here $A^{+}$denotes the Moore-Penrose pseudo-inverse of $A$, i.e. $x=A^{+} b$ satisfies $\|A x-b\|_{2}=\min , A \in \operatorname{Mat}(n), x, b \in \mathbb{R}^{n}$. Let $\xi_{0}=\left(\xi_{0}^{r}, \xi_{0}^{a}\right)$ be the drift velocity of the relative equilibrium $\operatorname{SE}(3) x_{0}$ at $x_{0}$ and assume that $\xi_{0}^{r} \neq 0$. Without loss of generality, choose $x_{0}$ in its group orbit such that $\xi_{0}^{r} \in \operatorname{so}(3) \simeq \mathbb{R}^{3}$ (see part b) for this identification) is parallel to $\xi_{0}^{a}$ and to $e_{3}$, and write $\xi_{0}^{r}=\omega_{0}^{\text {rot }} e_{3}$, where $\omega_{0}^{\text {rot }} \neq 0$. Align the family of relative equilibria $\operatorname{SE}(3) x_{\mathrm{RE}}(\mu)$ with $x_{\mathrm{RE}}(0)=x_{0}$ such that their drift velocity $\xi_{\mathrm{RE}}(\mu)$ at $x_{\mathrm{RE}}(\mu)$ also satisfies $\xi_{\mathrm{RE}}^{r}(\mu)=\omega^{\mathrm{rot}}(\mu) e_{3}$. If the Hopf frequency $\omega_{0}^{\text {Hopf }}$ satisfies $\omega_{0}^{\text {rot }} / \omega_{0}^{\text {Hopf }} \in \mathbb{Z}$ and this resonance is passed transversely as in (2.8), then, as in part $\mathrm{b})$, there is a curve $\mathcal{P}(s)$ of relative periodic orbits through $x(s) \approx x_{0}$ with drift symmetry $\gamma(s)=(R(s), a(s))$ at $x(s)$ satisfying $\phi_{3}(s)=0 \bmod 2 \pi$. Here $R(s)$ is determined by $\phi_{i}(s), i=1,2,3$, as in (2.9). These RPOs rotate and translate along a vector in the $\left(x_{1}, x_{2}\right)$-plane. The point around which they rotate approaches infinity as $s \rightarrow 0$.

d) Note that for the groups $\Gamma=\mathrm{SE}(2), \Gamma=\mathrm{SO}(3)$ and $\Gamma=\mathrm{SE}(3)$ considered above, resonance drift can only occur near relative equilibria with non-vanishing rotational velocity. Otherwise the linear map $\operatorname{ad}_{\xi_{0}}$ has no eigenvalues in $i \mathbb{R} \backslash\{0\}$, but this is necessary for resonance drift, c.f. part a) and [31].

\section{Dynamics near Hamiltonian relative equilibria}

As in the dissipative case, the meandering transition in Hamiltonian systems is studied by analyzing the equations near relative equilibria (2.3). Therefore in this section symmetric Hamiltonian systems and the structure of the equations (2.3) for Hamiltonian systems are reviewed. Then these results are applied to Hamiltonian systems with Euclidean symmetry, for later use in the analysis of the Hamiltonian meandering transition. Most of the material of this section is taken from $[12,17,26,28]$.

\subsection{Symmetric Hamiltonian systems}

In this section a brief introduction to symmetric Hamiltonian differential equations is given (see e.g. $[12,17]$ for more details). The starting point is a Hamiltonian ordinary differential equation on a smooth finite-dimensional symplectic manifold $\mathcal{M}$ with a symplectic form (i.e., a nondegenerate, closed 2-form) $\Omega_{x}, x \in \mathcal{M}$. A Hamiltonian vector field

$$
\dot{x}=f_{H}(x)
$$

is generated by a smooth function (the Hamiltonian) $H: \mathcal{M} \mapsto \mathbb{R}$ via the relationship

$$
\Omega_{x}\left(f_{H}(x), v\right)=\mathrm{D} H(x) v \quad x \in \mathcal{M}, v \in \mathcal{T}_{x} \mathcal{M} .
$$

Example 3.1 The simplest example is a Hamiltonian system

$$
\dot{x}=J^{D_{x}} H(x)
$$

on $\mathcal{M}=\mathbb{R}^{2 n}$, where

$$
\mathbb{J}=\left(\begin{array}{cc}
0 & \text { id } \\
- \text { id } & 0
\end{array}\right)
$$

and $H: \mathcal{M} \rightarrow \mathbb{R}$ is a smooth Hamiltonian. Then the symplectic form $\Omega$ is the standard symplectic form given by $\Omega(u, v)=\left\langle\mathbb{J}^{-1} u, v\right\rangle$, and $\mathbb{J}$ is called the symplectic structure matrix. By the Darboux-theorem, see e.g. [17], locally every Hamiltonian system has this form in suitable coordinates. 
Let us assume that a finite dimensional Lie group $\Gamma$ acts symplectically on $\mathcal{M}$, i.e., that

$$
\Omega_{\gamma x}(\gamma u, \gamma v)=\Omega_{x}(u, v) \quad \text { for all } x \in \mathcal{M}, \gamma \in \Gamma, u, v \in \mathcal{T}_{x} \mathcal{M}
$$

If $H$ is invariant under the action of $\Gamma$ then the vector field $f_{H}$ is $\Gamma$-equivariant.

Let $\mathbf{g}^{*}$ denote the dual of the Lie algebra $\mathbf{g}$ of $\Gamma$. By Noether's Theorem, for each continuous symmetry $\xi \in \mathbf{g}$ locally there is a conserved quantity $\mathbf{J}(\xi)(\cdot)$ of $(3.1)$. The function $\mathbf{J}(\xi)$ is linear in $\xi$, so that $\mathbf{J}$ maps into $\mathbf{g}^{*}$ (see e.g. [17]). It is assumed that $\mathbf{J}$ exists globally on $\mathcal{M}$.

Example 3.2 The dynamics of $N$ point vortices $\left(z_{1}, \ldots, z_{N}\right)=\left(x_{1}, y_{1}, \ldots, x_{N}, y_{N}\right) \in \mathbb{R}^{2 N}$ on the plane is given by the following Hamiltonian system $[1,2,21]$ :

$$
k_{i} \dot{x}_{i}=\frac{\partial H}{\partial y_{i}}, \quad k_{i} \dot{y}_{i}=-\frac{\partial H}{\partial x_{i}}, \quad i=1, \ldots, N,
$$

where $k_{i} \neq 0, k=1, \ldots, N$. The Hamiltonian $H$

$$
H\left(z_{1}, \ldots, z_{N}\right)=-\frac{1}{\pi} \sum_{\substack{i, j=0 \\ i<j}}^{N} k_{i} k_{j} \ln \left|z_{i}-z_{j}\right|
$$

of (3.3) is invariant under the action of the special Euclidean group of the plane $\Gamma=\mathrm{SE}(2)=$ $\mathrm{SO}(2) \ltimes \mathbb{R}^{2}$ on $\mathbb{R}^{2 N}$, given by

$$
\left(R_{\varphi}, a\right) \cdot\left(z_{1}, \ldots, z_{N}\right):=\left(R_{\varphi} z_{1}+a, \ldots, R_{\varphi} z_{N}+a\right)
$$

for $R_{\varphi} \in \mathrm{SO}(2)$ and $a \in \mathbb{R}^{2}$. The symplectic form

$$
\Omega\left(z_{1}, \ldots, z_{N}\right)=\sum_{i=1}^{N} k_{i} \mathrm{~d} x_{i} \wedge \mathrm{d} y_{i}
$$

is $\mathrm{SE}(2)$-invariant. The Hamiltonian system (3.3) can be obtained from Euler's equations for ideal fluids by modelling the point vortices as $\delta$-distributions, see e.g. [2]. In this example the space of momenta is $\mathbf{g}^{*}=\operatorname{se}(2)^{*}=\operatorname{so}(2)^{*} \oplus\left(\mathbb{R}^{2}\right)^{*}$. By Noether's Theorem, $\mathbf{J}(x)=\left(\mathbf{J}^{\phi}(x), \mathbf{J}^{a}(x)\right)$ is conserved. Here the angular momentum $\mathbf{J}^{\phi}$ and linear momentum $\mathbf{J}^{a}=\left(\mathbf{J}^{a_{1}}, \mathbf{J}^{a_{2}}\right)$ are given by

$$
\mathbf{J}^{\phi}(x)=-\frac{1}{2} \sum_{i=1}^{N} k_{i}\left|z_{i}\right|^{2}, \quad \mathbf{J}^{a_{1}}=\sum_{i=1}^{N} k_{i} y_{i}, \quad \mathbf{J}^{a_{2}}=-\sum_{i=1}^{N} k_{i} x_{i} .
$$

In the following transitions from relative equilibria to relative periodic orbits are studied when the conserved quantities angular and linear momentum are varied. In contrast to dissipative systems external parameters are not needed for the study of bifurcations. These transitions are studied by analyzing the symmetry reduced equations (2.3) for Hamiltonian systems. As in the general case, see Section 2.1, the reduction by the symmetry group is achieved by transforming the dynamics into a comoving frame. As a consequence, in the symmetry-reduced system the momentum is moving with the velocity of the comoving frame and might not be conserved any more. Therefore, to compute the reduced system in the Hamiltonian case, first the action of the symmetry group on the space of momenta is investigated.

\subsection{Symmetries of momentum maps}

Let us assume that $\mathbf{J}$ commutes with $\gamma \in \Gamma$,

$$
\mathbf{J}(\gamma x)=\gamma \mathbf{J}(x), \quad \gamma \in \Gamma,
$$


and, unless otherwise stated, that the action on momentum space $\mathbf{g}^{*}$ is the coadjoint action, so that the momentum map is $\mathrm{Ad}^{*}$-equivariant:

$$
\gamma \mathbf{J}(x)=\left(\operatorname{Ad}_{\gamma}^{*}\right)^{-1} \mathbf{J}(x), \quad \gamma \in \Gamma .
$$

The coadjoint action of $\Gamma$ on $\mathbf{g}^{*}$ is given by $\gamma \mu:=\left(\operatorname{Ad}_{\gamma}^{*}\right)^{-1} \mu$, where $\operatorname{Ad}_{\gamma}: \mathbf{g} \rightarrow \mathbf{g}$ from (2.2) is the adjoint action. The infinitesimal coadjoint action of $\mathbf{g}$ on $\mathbf{g}^{*}$ is defined by

$$
\xi \mu=-\operatorname{ad}_{\xi}^{*} \mu
$$

with $\operatorname{ad}_{\xi}$ as in (2.2). The isotropy subgroup of $\mu \in \mathbf{g}^{*}$ is denoted by

$$
\Gamma_{\mu}=\left\{\gamma \in \Gamma, \operatorname{Ad}_{\gamma}^{*} \mu=\mu\right\}
$$

and its Lie algebra by $\mathbf{g}_{\mu}$.

Example 3.3 As example the adjoint and coadjoint action for the Euclidean group are computed. They are needed later on for the computation of the drift dynamics near Hamiltonian rotating waves.

Let $\gamma=(\phi, a), \hat{\gamma}=(\hat{\phi}, \hat{a})$. Then

$$
\begin{aligned}
\gamma \hat{\gamma} \gamma^{-1} & =(\phi, a)(\hat{\phi}, \hat{a})(\phi, a)^{-1}=\left(\phi+\hat{\phi}, R_{\phi} \hat{a}+a\right)(\phi, a)^{-1} \\
& =\left(\phi+\hat{\phi}, R_{\phi} \hat{a}+a\right)\left(-\phi,-R_{-\phi} a\right)=\left(\hat{\phi},-R_{\hat{\phi}} a+R_{\phi} \hat{a}+a\right) \\
& =\left(\hat{\phi}, R_{\phi} \hat{a}+\left(\mathrm{id}-R_{\hat{\phi}}\right) a\right) .
\end{aligned}
$$

Letting $\hat{\phi}=\xi^{\phi} \epsilon, \hat{a}=\xi^{a} \epsilon$ and differentiating with respect to $\epsilon$ at $\epsilon=0$ one gets, with $\xi=$ $\left(\xi^{\phi}, \xi^{a}\right)=\left(\xi^{\phi}, \xi_{1}^{a}, \xi_{2}^{a}\right) \in \mathbb{R}^{3}$

$$
\operatorname{Ad}_{\gamma} \xi=\gamma \xi \gamma^{-1}=(\phi, a)\left(\xi^{\phi}, \xi^{a}\right)(\phi, a)^{-1}=\left(\xi^{\phi},\left(R_{\phi} \xi^{a}\right)_{1}+\xi^{\phi} a_{2},\left(R_{\phi} \xi^{a}\right)_{2}-\xi^{\phi} a_{1}\right) .
$$

Using $\operatorname{ad}_{\xi}=\left.\frac{\mathrm{d}}{\mathrm{d} t} \operatorname{Ad}_{\exp (t \xi)}\right|_{t=0}$, the adjoint actions of $\mathrm{SE}(2)$ on $\operatorname{se}(2)$ and the infinitesimal adjoint action of $\operatorname{se}(2)$ on $\mathrm{se}(2)$ are obtained:

$$
\operatorname{Ad}_{\gamma}=\left(\begin{array}{ccc}
1 & 0 & 0 \\
a_{2} & \cos \phi & -\sin \phi \\
-a_{1} & \sin \phi & \cos \phi
\end{array}\right), \quad \operatorname{ad}_{\xi}=\left(\begin{array}{ccc}
0 & 0 & 0 \\
\xi_{2}^{a} & 0 & -\xi^{\phi} \\
-\xi_{1}^{a} & \xi^{\phi} & 0
\end{array}\right) .
$$

The coadjoint action of $\mathrm{SE}(2)$ and $\mathrm{se}(2)$ and the infinitesimal coadjoint action of $\operatorname{se}(2)$ on $\mathrm{se}(2)^{*}$ are obtained by transposing and inverting $\mathrm{Ad}_{\gamma}$ and by transposition and multiplication by -1 of $\operatorname{ad}_{\xi}$ :

$$
\left(\operatorname{Ad}_{\gamma}^{*}\right)^{-1}=\left(\begin{array}{ccc}
1 & -\left(R_{-\phi} a\right)_{2} & \left(R_{-\phi} a\right)_{1} \\
0 & \cos \phi & -\sin \phi \\
0 & \sin \phi & \cos \phi
\end{array}\right), \quad-\operatorname{ad}_{\xi}^{*}=\left(\begin{array}{ccc}
0 & -\xi_{2}^{a} & \xi_{1}^{a} \\
0 & 0 & -\xi^{\phi} \\
0 & \xi^{\phi} & 0
\end{array}\right)
$$

From these equations it can be seen that the isotropy subgroup $\Gamma_{\mu}=\left\{\gamma \in \Gamma,\left(\operatorname{Ad}_{\gamma}^{*}\right)^{-1} \mu=\mu\right\}$ of $\mu \in \operatorname{se}(2)^{*}$ is $\Gamma_{\mu}=\Gamma=\operatorname{SE}(2)$ if and only if $\mu^{a}=0$ and that $\Gamma_{\mu} \simeq \mathbb{R}$ for $\mu^{a} \neq 0$.

Remark 3.4 In the case of zero total circulation $\mathcal{K}:=\sum_{i=1}^{N} k_{i}=0$ the momentum map $\mathbf{J}$ for the planar vortex dynamics from (3.4) is $\mathrm{Ad}^{*}$-equivariant, see [2]; but, if $\mathcal{K} \neq 0$, then, instead of (3.6), the equivariance condition (3.5) now holds for the action

$$
\gamma \cdot \kappa \mu:=\operatorname{Ad}_{\gamma^{-1}}^{*} \mu+\kappa(\gamma)
$$

of $\Gamma$ on $\mathbf{g}^{*}$. Here

$$
\kappa(\phi, a)=\mathcal{K}\left(-\frac{1}{2}|a|^{2}, a_{2},-a_{1}\right) \in \operatorname{se}(2)^{*}
$$


is called a cocycle, see [17]. In other words, (3.5) now becomes

$$
\mathbf{J}(\gamma x)=\gamma \cdot{ }_{\kappa} \mathbf{J}(x), \quad \text { for all } \gamma \in \Gamma .
$$

The infinitesimal cocycle $K: \mathbf{g} \times \mathbf{g} \rightarrow \mathbb{R}$ corresponding to the cocycle $\kappa$ is defined as

$$
K(\xi)=\left.\frac{\mathrm{d}}{\mathrm{d} t} \kappa\left(e^{t \xi}\right)\right|_{t=0} \in \mathbf{g}^{*},
$$

and in this case it is given by

$$
K(\xi, \eta)=\mathcal{K}\left\langle\xi^{a},\left(\begin{array}{cc}
0 & -1 \\
1 & 0
\end{array}\right) \eta_{a}\right\rangle=\mathcal{K}\left(-\xi_{1}^{a} \eta_{2}^{a}+\xi_{2}^{a} \eta_{1}^{a}\right) .
$$

The infinitesimal action of the Lie algebra $\mathbf{g}$ of $\Gamma$ on $\mathbf{g}^{*}$ is defined as

$$
\xi \cdot K \mu=\left.\frac{\mathrm{d}}{\mathrm{d} t} \exp (t \xi) \cdot_{\kappa} \mu\right|_{t=0}=-\operatorname{ad}_{\xi}^{*} \mu+K(\xi) .
$$

The isotropy subgroup of $\mu \in \mathbf{g}^{*}$ with respect to the cocycle action (3.10) is denoted by $\Gamma_{\mu}^{\kappa}$. Its Lie algebra is denoted by $\mathbf{g}_{\mu}^{K}=\mathcal{T}_{\mathrm{id}} \Gamma_{\mu}^{\kappa}$. For later use, note that $\gamma=(\phi, a) \in \Gamma_{\mu}^{\kappa}$ for $\mu \in \operatorname{se}(2)^{*}$ if and only if

$$
\left(R_{\phi}-\mathrm{id}\right) \mu^{a}=\mathcal{K}\left(\begin{array}{c}
-a_{2} \\
a_{1}
\end{array}\right) .
$$

Hence for non-vanishing cocycle the isotropy subgroup of every $\mu \in \mathbf{g}^{*}$ is conjugate to $\mathrm{SO}(2)$.

Let $x_{0}$ lie on a relative equilibrium $\Gamma x_{0}$ with drift velocity $\xi_{0} \in \mathbf{g}$ at $x_{0}$, so that $\Phi_{t}\left(x_{0}\right)=$ $\exp \left(t \xi_{0}\right) x_{0}$. Since momentum is conserved,

$$
\mu_{0}=\mathbf{J}\left(x_{0}\right)=\mathbf{J}\left(\Phi_{t}\left(x_{0}\right)\right)=\mathbf{J}\left(\exp \left(t \xi_{0}\right) x_{0}\right)=\exp \left(t \xi_{0}\right) \mu_{0},
$$

and therefore $\mu_{0}$ is fixed by $\xi_{0}$ :

$$
\xi_{0} \mu_{0}=0 .
$$

Such pairs $(\xi, \mu) \in \mathbf{g} \oplus \mathbf{g}^{*}$ are called velocity-momentum pairs. Note that the action of $\xi_{0}$ on $\mu_{0}$ in (3.16) is the infinitesimal coadjoint action (3.7), or the infinitesimal action with cocycle (3.14), depending on the symmetry property of the momentum map.

Similarly, if $x_{0}=\gamma_{0}^{-1} \Phi_{T_{0}}\left(x_{0}\right)$ lies on a relative periodic orbit with drift symmetry $\gamma_{0}$ and momentum $\mu_{0}=\mathbf{J}\left(x_{0}\right)$ then $\mu_{0}$ is fixed by $\gamma_{0}$ :

$$
\gamma_{0} \mu_{0}=\mu_{0} .
$$

Such pairs $(\gamma, \mu) \in \Gamma \times \mathbf{g}^{*}$ are called drift-momentum pairs.

\subsection{Dynamics near Hamiltonian relative equilibria}

For a symplectic manifold $\mathcal{M}$ with $\mathrm{Ad}^{*}$-equivariant momentum map the normal space $\mathcal{N}$ to the group orbit $\Gamma x_{0}$ at $x_{0} \in \mathcal{M}$ from Section 2.1 can be decomposed as:

$$
\mathcal{M} / \Gamma \simeq \mathcal{N}=\mathcal{N}_{0} \oplus \mathcal{N}_{1} \cong \mathbf{g}_{\mu_{0}}^{*} \oplus \mathcal{N}_{1}
$$

Here

$$
\mathbf{g}_{\mu_{0}}=\mathcal{T}_{\mathrm{id}} \Gamma_{\mu_{0}}=\left\{\xi \in \mathbf{g}: \operatorname{ad}_{\xi}^{*} \mu_{0}=0\right\}
$$

is the isotropy subalgebra of the momentum $\mu_{0}=\mathbf{J}\left(x_{0}\right)$ of $x_{0}$. The space $\mathcal{N}_{0}$ is isomorphic to a section transverse to the momentum group orbit $\Gamma \mu_{0}$ at $\mu_{0}$. To see that $\mathcal{N}_{0} \simeq \mathbf{g}_{\mu_{0}}^{*}$, let $\mathbf{n}_{\mu_{0}}$ be a complement to $\mathbf{g}_{\mu_{0}}$ in $\mathbf{g}$ and let $\operatorname{ann}\left(\mathbf{n}_{\mu_{0}}\right)$ denote the annihilator of $\mathbf{n}_{\mu_{0}}$ in $\mathbf{g}^{*}$. Then $\mathcal{T}_{\mu_{0}} \Gamma \mu_{0}=\mathbf{g} \mu_{0}=\operatorname{ann}\left(\mathbf{g}_{\mu_{0}}\right)$, and so $\operatorname{ann}\left(\mathbf{n}_{\mu_{0}}\right) \cong \mathbf{g}_{\mu_{0}}^{*}$ is a section transverse to $\Gamma \mu_{0}$ at $\mu_{0}$. The symplectic normal space or symplectic slice $\mathcal{N}_{1}$ at $x_{0}$ is a slice to the $\Gamma_{\mu_{0}}$ orbit of $x_{0}$ in the momentum level set $\mathbf{J}^{-1}\left(\mu_{0}\right)$, c.f. Figure 5. Moreover, there is a choice of slice $\mathcal{N}$ such that the coordinate transformation $x \rightarrow(\gamma, \nu, w) \in \Gamma \times \mathcal{N}_{0} \oplus \mathcal{N}_{1}$, where $x$ lies in some 


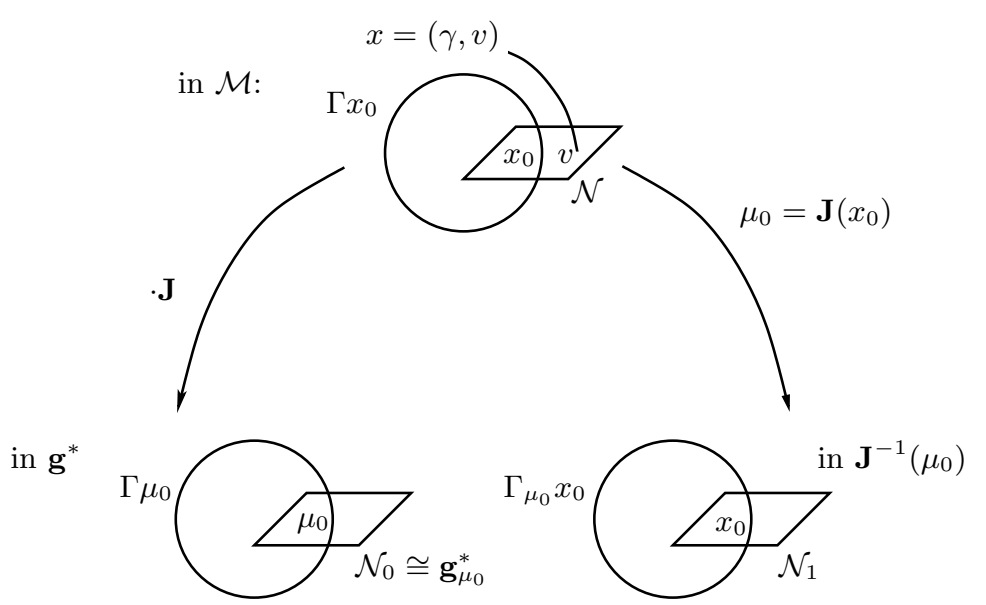

Figure 5: Symplectic Slice Theorem

$\Gamma$-invariant neighbourhood $\mathcal{U}$ of $\Gamma x_{0}$, is symplectic with symplectic form $\Omega_{\Gamma \times \mathcal{N}}$ on $\Gamma \times \mathcal{N}$ given by $\Omega_{\Gamma \times \mathcal{N}}=\Omega_{\Gamma \times \mathbf{g}_{\mu_{0}}^{*}}+\Omega_{\mathcal{N}_{1}}$. Here $\Omega_{\mathcal{N}_{1}}$ is the symplectic form on $\mathcal{N}_{1}$ and $\Omega_{\Gamma \times \mathbf{g}_{\mu_{0}}^{*}}$ the symplectic form on $\Gamma \times \mathbf{g}_{\mu_{0}}^{*}$, obtained by restriction of the symplectic form on $\mathcal{T}^{*} \Gamma \simeq \Gamma \times \mathbf{g}^{*}$. In these coordinates the momentum map becomes

$$
\mathbf{J}(\gamma, \nu, w)=\gamma\left(\mu_{0}+\nu\right),
$$

see $[11,16]$ and also $[26]$. Let $\mathbb{J}_{\mathcal{N}_{1}}$ be the structure matrix of the symplectic form on $\mathcal{N}_{1}$.

One more technical assumption is needed: In this paper, unless otherwise stated, it is assumed that $\mu_{0}$ is split, i.e., there is a $\Gamma_{\mu_{0}}^{\mathrm{id}}$-invariant complement to $\mathbf{g}_{\mu_{0}}$ in $\mathbf{g}$. Here $\Gamma_{\mu_{0}}^{\mathrm{id}}$ is the identity component of $\Gamma_{\mu_{0}}$. This condition is always satisfied for compact groups and also for the special Euclidean group of the plane, see [26]. For the general case see [26].

Theorem 3.5 [26, Theorem 3.1] Let, as above, $(\gamma, v), v=(\nu, w) \in \mathcal{N}, \gamma \in \Gamma$, parametrize a $\Gamma$-invariant neighbourhood $\mathcal{U}$ of the relative equilibrium $\Gamma x_{0}$ with momentum $\mu_{0}=\mathbf{J}\left(x_{0}\right)$. Let $h(\nu, w)$ be the restriction of the Hamiltonian $H$ to the slice $\mathcal{N}=\mathbf{g}_{\mu_{0}}^{*} \oplus \mathcal{N}_{1}$ and let $\mu_{0}$ be split. Assume that the momentum map is $\mathrm{Ad}^{*}$-equivariant. Then $\gamma(t) \in \Gamma, \nu(t) \in \mathbf{g}_{\mu_{0}}^{*}, w(t) \in \mathcal{N}_{1}$, where $x(t) \simeq(\gamma(t), \nu(t), w(t)) \in \mathcal{U}$ solves (3.1), satisfy the differential equations

$$
\dot{\gamma}=\gamma \mathrm{D}_{\nu} h(\nu, w), \quad \dot{\nu}=\operatorname{ad}_{\mathrm{D}_{\nu} h(\nu, w)}^{*} \nu, \quad \dot{w}=\mathbb{J}_{\mathcal{N}_{1}} \mathrm{D}_{w} h(\nu, w) .
$$

As in the non-Hamiltonian case, the relative equilibrium $\Gamma x_{0}$ corresponds to the equilibrium $v=(0,0) \in \mathcal{N}$ of the slice equation on $\mathcal{N}$. The first equation describes the motion of the body frame. Here $\mathrm{D}_{\nu} h$ is the velocity of the body frame, and $\mathrm{D}_{\nu} h(0,0)=\xi_{0}$ is the drift velocity of the relative equilibrium $\Gamma x_{0}$ at $x_{0}$. The second equation describes the motion of the momenta in body coordinates and the last equation models the shape dynamics.

From a comparison of (3.19) with the equations near relative equilibria in the general case (2.3), it becomes apparent that in the Hamiltonian case $v=(\nu, w)$ and

$$
f_{\Gamma}(v)=\mathrm{D}_{\nu} h(\nu, w), \quad f_{\mathcal{N}}(v)=\left(\begin{array}{c}
\operatorname{ad}_{\mathrm{D}_{\nu} h}^{*}(\nu) \\
\mathbb{J}_{\mathcal{N}_{1}} \mathrm{D}_{w} h(\nu, w)
\end{array}\right) .
$$

So the slice equation $\dot{v}=f_{\mathcal{N}}(v)$ now consists of the two differential equations for $\dot{\nu}$ and $\dot{w}$. Moreover

$$
\mathrm{D} f_{\mathcal{N}}(0)=\left(\begin{array}{cc}
\left.\operatorname{ad}_{\xi_{0}}^{*}\right|_{\mathbf{g}_{\mu_{0}}^{*}} ^{*} & 0 \\
\mathbb{J}_{\mathcal{N}_{1}} \mathrm{D}_{\nu w}^{2} h(0) & \mathbb{J}_{\mathcal{N}_{1}} \mathrm{D}_{w}^{2} h(0)
\end{array}\right) .
$$

The energy $h(\nu, w)$ is a conserved quantity of the slice equation which is a Poisson-system, see [17]. Any function $C(\nu, w)$ which is a conserved quantity of the slice equation for all choices 
of Hamiltonians $h(\nu, w)$ is called a Casimir of the slice equation. Note that the flow-invariant symplectic leaves of the slice equation are given by $\Gamma_{\mu_{0}}^{\mathrm{id}} \nu_{0} \times \mathcal{N}_{1}$ where $\nu_{0} \in \mathcal{N}_{0}$.

Note for later reference that (3.20) is true for non-split $\mu$ as well if the infinitesimal coadjoint action ad $\mathrm{d}_{\xi}^{*}$ restricted to $\operatorname{ann}\left(\mathbf{n}_{\mu_{0}}\right) \simeq \mathbf{g}_{\mu_{0}}^{*}$ in $\left.\operatorname{ad}_{\xi_{0}}^{*}\right|_{\mathbf{g}_{\mu_{0}}^{*}}$ is replaced by the corresponding action on $\mathbf{g}_{\mu_{0}}$. For split $\mu_{0}$, both these actions coincide, see [26].

Remark 3.6 With the notation from (3.7), the $\dot{\nu}$-equation can be rewritten as

$$
\dot{\nu}=-\mathrm{D}_{\nu} h(\nu, w) \nu .
$$

As shown in [28], Theorem 3.5 remains true in the case of a momentum map which is symmetric with respect to a cocycle action, if the infinitesimal coadjoint action in the $\dot{\nu}$-equation is replaced by the corresponding action (3.14) with a cocycle. Then the $\dot{\nu}$-equation becomes

$$
\dot{\nu}=-\mathrm{D}_{\nu} h(\nu, w) \cdot_{K} \nu
$$

provided that $\mu_{0}$ is split for the action of $\Gamma^{\kappa}$ on $\mathbf{g}$, i.e., if there is a $\left(\Gamma_{\mu_{0}}^{\kappa}\right)^{\text {id }}$-invariant complement $\mathbf{n}_{\mu_{0}}^{K}$ to $\mathbf{g}_{\mu_{0}}^{K}$ in $\mathbf{g}$, where $\left(\Gamma_{\mu_{0}}^{\kappa}\right)^{\text {id }}$ is the identity component of $\Gamma_{\mu_{0}}^{\kappa}$. Moreover, as before, $\mathcal{N}_{0} \simeq$ $\operatorname{ann}\left(\mathbf{n}_{\mu_{0}}^{K}\right) \simeq\left(\mathbf{g}_{\mu_{0}}^{K}\right)^{*}$.

Remark 3.7 For later use, let us consider parameter dependent Hamiltonian systems

$$
\dot{x}=f(x, \mathcal{K}),
$$

where $f$ is defined by

$$
\Omega(x, \mathcal{K})(f(x, \mathcal{K}), v)=\mathrm{D}_{x} H(x, \mathcal{K}) v \quad x \in \mathcal{M}, v \in \mathcal{T}_{x} \mathcal{M} .
$$

Assume that the symplectic form $\Omega(\mathcal{K})$, the Hamiltonian $H(\cdot, \mathcal{K})$ and the momentum map $\mathbf{J}(\cdot, \mathcal{K})$ depend smoothly on a parameter $\mathcal{K}$. Then Theorem 3.5 still applies, and the sections $\mathcal{N}_{0}(\mathcal{K})$, $\mathcal{N}_{1}(\mathcal{K})$, as well as the Hamiltonian $h(\nu, w, \mathcal{K})$, depend smoothly on $\mathcal{K}$, as long as the dimensions of $\mathcal{N}_{0}(\mathcal{K})$ and $\mathcal{N}_{1}(\mathcal{K})$ are constant. See the proofs in $[11,16]$ and $[26]$.

Example 3.8 For later use, let us derive the differential equations (3.19) near rotating waves of Hamiltonian systems (3.1) with symmetry group $\Gamma=\mathrm{SE}(2)$, in the case of an $\mathrm{Ad}^{*}$-equivariant momentum map. Let $x_{0}$ lie on a rotating wave so that $\Phi_{t}\left(x_{0}\right)=\exp \left(t \xi_{0}\right) x_{0}, \xi_{0} \in \operatorname{so}(2)$, $\mu_{0}=\mathbf{J}\left(x_{0}\right)$. From (3.16) it follows that $0=-\operatorname{ad}_{\xi_{0}}^{*} \mu_{0}$, with ad $\xi_{\xi_{0}}^{*}$ from (3.9). Therefore $\mu_{0}^{a}=0$, so that the rotating wave through $x_{0}$ has vanishing linear momentum. Then $\Gamma_{\mu_{0}}=\mathrm{SE}(2)$, see Example 3.3. Hence $\mathcal{N}_{0} \simeq \mathbf{g}_{\mu_{0}}^{*} \simeq \operatorname{se}(2)^{*}$, and so the equations (3.19) for $\operatorname{SE}(2)$-equivariant Hamiltonian systems are

$$
\left.\begin{array}{rl}
\dot{\phi} & =\mathrm{D}_{\nu^{\phi}} h, \quad \dot{a}=R_{\phi} \mathrm{D}_{\nu^{a}} h, \\
\dot{\nu}^{\phi} & =\nu_{1}^{a} \mathrm{D}_{\nu_{2}^{a}} h(\nu, w)-\nu_{2}^{a} \mathrm{D}_{\nu_{1}^{a}} h(\nu, w) \\
\dot{\nu}_{1}^{a} & =\nu_{2}^{a} \mathrm{D}_{\nu^{\phi}} h(\nu, w) \\
\dot{\nu}_{2}^{a} & =-\nu_{1}^{a} \mathrm{D}_{\nu^{\phi}} h(\nu, w) \\
\dot{w} & =\mathbb{J}_{\mathcal{N}_{1}} \mathrm{D}_{w} h(\nu, w)
\end{array}\right\} \Leftrightarrow \quad \dot{\gamma}=\gamma \mathrm{D}_{\nu} h(\nu, w)
$$

Example 3.9 Also for later use, let us consider the equations (3.21) for Hamiltonian systems (3.22) which have a momentum map $\mathbf{J}(\cdot, \mathcal{K})$ with cocycle $(3.11), \mathcal{K} \neq 0$. From (3.10) and (3.11) it follows that the momentum $\mu_{0}$ can always be translated such that $\mu_{0}^{a}=0$. For $\mu_{0}^{a}=0$ one has $\xi=\left(\xi^{\phi}, \xi^{a}\right) \in \mathbf{g}_{\mu_{0}}^{K}$ if and only if $\xi^{a}=0$, and so

$$
\Gamma_{\mu_{0}}^{\kappa}=\mathrm{SO}(2) \times\{0\} \subseteq \mathrm{SE}(2) .
$$

For $\xi^{a}=0$ the infinitesimal cocycle (3.13) vanishes. Hence $\left\{\left(0, \xi^{a}\right) \in \operatorname{se}(2), \xi^{a} \in \mathbb{R}^{2}\right\}$ is a $\Gamma_{\mu_{0}}^{\kappa}$ invariant complement to $\mathbf{g}_{\mu_{0}}^{K}=\operatorname{so}(2)$ in se(2), and $\mu_{0}$ is split. Consequently, $\mathcal{N}_{0} \simeq\left(\mathbf{g}_{\mu_{0}}^{K}\right)^{*}=$ so $(2)^{*}$, and $\dot{\nu}=0$ in $(3.21)$. 


\section{The meandering transition in Hamiltonian systems}

In this section a Hamiltonian analogue of the meandering transition for dissipative systems, which was described in Section 2.2 above, is presented, using the equations near Hamiltonian relative equilibria (3.19) from Section 3. First Euclidean symmetric Hamiltonian systems with an $\mathrm{Ad}^{*}$-equivariant momentum map are studied (Sections 4.1 - 4.3). Then systems with Euclidean symmetry for which the momentum map has a cocycle are considered (Section 4.4).

\subsection{Persistence of rotating waves}

In this section and in the following sections $4.2-4.3$ it is assumed that (3.1) has the symmetry group $\Gamma=\mathrm{SE}(2)$ and an $\mathrm{Ad}^{*}$-equivariant momentum map.

As a prerequisite for the analysis of the transition from rotating waves to modulated rotating and modulated traveling waves, the persistence of nondegenerate rotating waves to nearby momentum values is studied.

Definition 4.1 A relative equilibrium $\Gamma x_{0}$ of (3.1) is called nondegenerate if $\mathrm{D}_{w}^{2} h(0)$ is invertible. Here $h(\nu, w)$ is the Hamiltonian in the symmetry adapted coordinates near $x_{0}$ from (3.19).

Note that a relative equilibrium is typically nondegenerate. The next proposition shows that nondegenerate rotating waves persist to nearby angular momentum.

Proposition 4.2 Let $\mathrm{SE}(2) x_{0}$ be a nondegenerate rotating wave of an $\mathrm{SE}(2)$-equivariant Hamiltonian system (3.1) with $\mathrm{Ad}^{*}$-equivariant momentum map $\mathbf{J}(\cdot)$, and let $\mu_{0}=\mathbf{J}\left(x_{0}\right)$. Then there is a one-parameter family $\mathrm{SE}(2) x_{\mathrm{RW}}\left(\nu^{\phi}\right)$ of rotating waves nearby parametrized by angular momentum $\mu^{\phi}=\mu_{0}^{\phi}+\nu^{\phi}$ with vanishing linear momentum such that $x_{\mathrm{RW}}\left(\nu^{\phi}\right) \simeq$ $\left(\mathrm{id},\left(\nu^{\phi}, 0,0\right)^{T}, w_{\mathrm{RW}}\left(\nu^{\phi}\right)\right)$ is smooth in $\nu^{\phi}$ and $x(0)=x_{0}, w_{\mathrm{RW}}(0)=0$.

Proof. Rotating waves are equilibria of the slice equation, i.e., of the $(\nu, w)$-system of (3.23). Using the nondegeneracy assumption, the equation $0=\dot{w}=\mathbb{J}_{\mathcal{N}_{1}} \mathrm{D}_{w} h(\nu, w)$ can be solved by the implicit function theorem for $w_{\mathrm{RW}}(\nu)$ such that $w_{\mathrm{RW}}(0)=0$. For rotating waves the linear momentum has to vanish, see Example 3.8. Hence $\nu^{a}=0$ in any rotating wave. From the $\dot{\nu}$-equation of (3.23) then $\dot{\nu}^{\phi}=0, \dot{\nu}^{a}=0$ follows. Hence $x_{\mathrm{RW}}\left(\nu^{\phi}\right) \simeq\left(\mathrm{id},\left(\nu^{\phi}, 0,0\right)^{T}, w_{\mathrm{RW}}\left(\nu^{\phi}\right)\right)$ lies on a rotating wave of (3.1) for all $\nu^{\phi} \approx 0$. By (3.18), $\mathbf{J}\left(x_{\mathrm{RW}}\left(\nu^{\phi}\right)\right)=\mu_{0}+\nu=\left(\mu_{0}^{\phi}+\nu^{\phi}, 0\right)$.

A persistence theory for teneric nondegenerate relative equilibria of Hamiltonian systems with general noncompact symmetry group has been developed in [32], see also [20] for an example of non-persistence of rotating waves to non-vanishing linear momentum in point vortex dynamics. The above proposition could also be proved by applying the results of [32] to the example $\Gamma=\mathrm{SE}(2)$. However, the direct proof given above is more elementary.

\subsection{Bifurcation of modulated travelling waves}

The following theorem states that typically rotating waves of Euclidean equivariant Hamiltonian systems with $\mathrm{Ad}^{*}$-equivariant momentum map persist to modulated traveling waves at nearby linear momenta $\mu^{a} \neq 0$. Consequently resonance drift occurs generically.

Theorem 4.3 Let $\mathrm{SE}(2) x_{0}$ be a nondegenerate rotating wave of an $\mathrm{SE}(2)$-equivariant Hamiltonian system (3.1) with $\mathrm{Ad}^{*}$-equivariant momentum map. Denote its rotation frequency by $\omega_{0}^{\text {rot }}=\xi_{0}^{\phi}$ and assume that $\omega_{0}^{\text {rot }} \neq 0$. If all eigenvalues $\mathrm{i} \omega_{0}$ of $\mathbb{J}_{\mathcal{N}_{1}} \mathrm{D}_{w}^{2} h(0)$ satisfy

$$
\omega_{0} / \omega_{0}^{\text {rot }} \notin \mathbb{Z}
$$


then the rotating wave $\mathrm{SE}(2) x_{0}$ persists as a modulated traveling wave $\mathcal{P}_{\mathrm{MTW}}\left(\nu^{\phi}, r^{a}\right)$ to all nearby momentum values $\mu=\left(\mu_{0}^{\phi}+\nu^{\phi}, \nu^{a}\right), r^{a}=\left\|\nu^{a}\right\|$. Moreover there is a smooth function $x_{\mathrm{MTW}}\left(\nu^{\phi}, r^{a}\right) \in \mathcal{P}_{\mathrm{MTW}}\left(\nu^{\phi}, r^{a}\right)$ such that $x_{\mathrm{MTW}}\left(\nu^{\phi}, 0\right)=x_{\mathrm{RW}}\left(\nu^{\phi}\right)$. Here $\operatorname{SE}(2) x_{\mathrm{RW}}\left(\nu^{\phi}\right)$ is the family of rotating waves from Proposition 4.2. The relative period $T_{\mathrm{MTW}}\left(\nu^{\phi}, r^{a}\right)$ of the modulated traveling wave $\mathcal{P}_{\mathrm{MTW}}\left(\nu^{\phi}, r^{a}\right)$ is close to $T_{\mathrm{MTW}}(0)=\frac{2 \pi}{\left|\omega_{0}^{\text {rot }}\right|}$, and the translation drift $\gamma_{\mathrm{MTW}}\left(\nu^{\phi}, r^{a}\right)=\left(0, a_{\mathrm{MTW}}\left(\nu^{\phi}, r^{a}\right)\right)$ of the modulated traveling wave at $x_{\mathrm{MTW}}\left(\nu^{\phi}, r^{a}\right)$ satisfies $a_{\mathrm{MTW}}\left(\nu^{\phi}, 0\right)=0$.

Proof. The rotating wave $\mathrm{SE}(2) x_{0}$ is treated as a periodic orbit of period $T_{0}=\frac{2 \pi}{\omega_{0}^{\text {rot }}}$. Introduce polar coordinates $\nu^{a}=\left(r^{a} \cos \phi^{a}, r^{a} \sin \phi^{a}\right)$. Then (3.23) implies that $r^{a}=\left\|\nu^{a}\right\|_{2}$ is a conserved quantity (Casimir) of the slice equation. Since the slice equation also conserves energy, the set

$$
\mathcal{N}_{E, r^{a}}=\left\{(\nu, w) \in \mathcal{N}, \quad h(\nu, w)=E, \quad\left\|\nu^{a}\right\|_{2}=r^{a}\right\}
$$

is flow-invariant. From $\dot{\phi}^{a}=\mathrm{D}_{\nu^{\phi}} h(\nu, w) \approx \omega_{0}^{\text {rot }} \neq 0$ for $(\nu, w) \approx 0$ it can be deduced that for $E \approx E_{0}=H\left(x_{0}\right), r^{a}>0, r^{a} \approx 0$, the section

$$
\mathcal{S}_{E, r^{a}}=\left\{(\nu, w) \in \mathcal{N}_{E, r^{a}}, \phi^{a}=0,(\nu, w) \approx 0\right\}
$$

is tranversal to the flow in $\mathcal{N}_{E, r^{a}}$. Let $\Pi\left(E, r^{a}, \cdot\right): \mathcal{S}_{E, r^{a}} \rightarrow \mathcal{S}_{E, r^{a}}$ be the Poincaré-map to the Poincaré-section $\mathcal{S}_{E, r^{a}}$. Since $\mathrm{D}_{(\nu, w)} h(0)=\left(\xi_{0}, 0\right)$ with $\xi_{0}^{\phi}=\omega_{0}^{\text {rot }} \neq 0$, the sections $\mathcal{S}_{E, r^{a}}$, $E \approx E_{0}, r^{a} \approx 0$, can be parametrized as

$$
\mathcal{S}_{E, r^{a}}=\left\{\left(\nu^{\phi}, w\right), \nu^{\phi}=\nu^{\phi}\left(E, r^{a}, w\right), w \in \mathcal{N}_{1}\right\} .
$$

Hence $\Pi\left(E, r^{a}, \cdot\right)$ can be considered as a map from $\mathcal{N}_{1}$ to itself. By assumption $k \mathrm{i} \omega_{0}^{\text {rot }}, k \in \mathbb{Z}$, is not an eigenvalue of $\mathbb{J}_{\mathcal{N}_{1}} \mathrm{D}_{w}^{2} h(0)$. Therefore

$$
\mathrm{D}_{w} \Pi\left(E_{0}, 0,0\right)-\mathrm{id}=\exp \left(\frac{2 \pi}{\left|\omega_{0}^{\text {rot }}\right|} \mathbb{J}_{\mathcal{N}_{1}} \mathrm{D}_{w}^{2} h(0)\right)-\mathrm{id}
$$

is invertible, and so there is a fixed point $w\left(E, r^{a}\right)$ of $\Pi\left(E . r^{a}, \cdot\right)$ for each $E \approx E_{0}, r^{a} \approx 0$. As $\mathrm{D}_{\nu^{\phi}} h(0)=\omega_{0}^{\text {rot }} \neq 0$, this family of fixed points can be parametrized by $\nu^{\phi}$ and $r^{a}$ instead of $E$ and $r^{a}$.

The periodic orbits of the slice equation through $v\left(\nu^{\phi}, r^{a}\right)=\left(\nu\left(\nu^{\phi}, r^{a}\right), w\left(\nu^{\phi}, r^{a}\right)\right)$, where $\nu\left(\nu^{\phi}, r^{a}\right)=\left(\nu^{\phi}, r^{a}, 0\right)$, correspond to relative periodic orbits $\mathcal{P}_{\mathrm{MTW}}\left(\nu^{\phi}, r^{a}\right)$ of $(3.1)$ through $x_{\mathrm{MTW}}\left(\nu^{\phi}, r^{a}\right) \simeq\left(\mathrm{id}, v\left(\nu^{\phi}, r^{a}\right)\right)$ with momentum

$$
\mathbf{J}\left(x_{\mathrm{MTW}}\left(\nu^{\phi}, r^{a}\right)\right)=\mu_{0}+\nu\left(\nu^{\phi}, r^{a}\right)=\left(\mu_{0}^{\phi}+\nu^{\phi}, r^{a}, 0\right),
$$

see (3.18). For vanishing linear momentum $r^{a}=0$ they reduce to the rotating waves $\mathrm{SE}(2) x_{\mathrm{RW}}\left(\nu^{\phi}\right)$ from Proposition 4.2.

By (3.17), any RPO with drift $\gamma=(\phi, a)$ and momentum $\mu$ satisfies $\left(\operatorname{Ad}_{\gamma}^{*}\right)^{-1} \mu=\mu$, with $\left(\operatorname{Ad}_{\gamma}^{*}\right)^{-1}$ as in (3.9). Because of (3.9), the condition $\mu^{a} \neq 0$ implies $\phi=0$ so that the relative periodic orbits $\mathcal{P}_{\mathrm{MTW}}\left(\nu^{\phi}, r^{a}\right)$ are modulated traveling waves for $r^{a} \neq 0$.

\subsection{Bifurcation of modulated rotating waves}

In this section the existence of modulated rotating waves near elliptic rotating waves is proved by the Lyapounov centre theorem.

Definition 4.4 A relative equilibrium $\Gamma x_{0}, x_{0} \simeq(\mathrm{id},(\nu, w)=(0,0))$, of a $\Gamma$-equivariant Hamiltonian system (3.1) is called elliptic if all eigenvalues of the linearization $\mathbb{J}_{\mathcal{N}_{1}} \mathrm{D}_{w}^{2} h(0)$ of the $\dot{w}$-dynamics of (3.19) lie in $i \mathbb{R} \backslash\{0\}$, and nonresonant if all its eigenvalues are simple and no eigenvalue $\mathrm{i} \omega_{j}$ is an integer multiple of another eigenvalue $\mathrm{i} \omega_{k}$ for $\omega_{j} \neq \omega_{k}$. 
Note that any stable relative equilibrium is elliptic and that relative equilibria are elliptic for an open range of parameters (until a Hamiltonian Hopf bifurcation of the $\dot{w}$-equation of (3.19) occurs).

Definition 4.5 Let $\Gamma x_{0}, x_{0} \simeq(\mathrm{id},(\nu, w)=(0,0))$, be an elliptic relative equilibrium of $(3.1)$, and denote the eigenvalues of $\mathbb{J}_{\mathcal{N}_{1}} \mathrm{D}_{w}^{2} h(0)$ by $\pm \mathrm{i} \omega_{j}, j=1, \ldots, d, d:=\frac{1}{2} \operatorname{dim} \mathcal{N}_{1}$. The signs of the normal frequencies $\omega_{j}$ are chosen such that

$$
h(0, w)=\sum_{j=1}^{d} \frac{\omega_{j}}{2}\left\langle w_{j}, w_{j}\right\rangle+O\left(\|w\|^{3}\right) .
$$

Here $w=\left(w_{1}, \ldots, w_{d}\right), w_{j} \in \mathbb{R}^{2}$, are suitable coordinates on $\mathcal{N}_{1}$. The sign of $\omega_{j}$ is called the Krein-signature of $\omega_{j}$. There is an $m: n$-resonance between the normal frequencies $\omega_{j}$ and $\omega_{k}$ if $m \omega_{j}=n \omega_{k}, m, n \in \mathbb{Z}$.

Proposition 4.6 Let $\mathrm{SE}(2) x_{0}$ be a non-resonant elliptic rotating wave of an $\mathrm{SE}(2)$-equivariant Hamiltonian system (3.1) with $\mathrm{Ad}^{*}$-equivariant momentum map. Let $\omega_{0}^{\text {rot }}$ be its rotation frequency, let $H\left(x_{0}\right)=E_{0}$ be its energy and let $\mu_{0}=\mathbf{J}\left(x_{0}\right)$ be its momentum at $x_{0}$. Denote the eigenvalues of $\mathbb{J}_{\mathcal{N}_{1}} \mathrm{D}_{w}^{2} h(0)$ by $\pm \mathrm{i} \omega_{j}, j=1, \ldots, \frac{1}{2} \operatorname{dim} \mathcal{M}-3$. Then there are $\left(\frac{1}{2} \operatorname{dim} \mathcal{M}-3\right)$ many two-dimensional families $\mathcal{P}_{j}\left(\nu^{\phi}, s\right)$ of $R P O s, j=1, \ldots, \frac{1}{2} \operatorname{dim} \mathcal{M}-3$, of (3.1), where $s \geq 0, \nu^{\phi} \approx 0$, with angular momentum $\mu_{0}^{\phi}+\nu^{\phi}$, with vanishing linear momentum, with energy $E=H\left(x_{\mathrm{RW}}\left(\nu^{\phi}\right)\right) \pm s^{2}$ (depending on the Krein signature of $\left.\omega_{j}\right)$, and with relative periods $T_{j}\left(\nu^{\phi}, s\right)$ such that $T_{j}(0,0)=2 \pi /\left|\omega_{j}\right|$. Moreover there are smooth functions $x_{j}\left(\nu^{\phi}, s\right)$ with $x_{j}\left(\nu^{\phi}, s\right) \in \mathcal{P}_{j}\left(\nu^{\phi}, s\right), x_{j}\left(\nu^{\phi}, 0\right)=x_{\mathrm{RW}}\left(\nu^{\phi}\right)$. If $\omega_{0}^{\text {rot }} / \omega_{j} \notin \mathbb{Z}$ for all normal frequencies $\omega_{j}$ then all these RPOs are proper modulated rotating waves for $\left(\nu^{\phi}, s\right) \approx 0$. Proper modulated rotating waves do not persist to non-zero linear momenta.

Proof. First note that proper modulated rotating waves have a drift symmetry $\gamma=(\phi, a)$ with $\phi \neq 0 \bmod 2 \pi$. This implies, because of (3.17) and (3.9), that the linear momentum $\nu^{a}$ of a proper modulated rotating wave vanishes. Equation $(3.23)$ implies that $\dot{\nu}^{\phi} \equiv 0, \dot{\nu}^{a} \equiv 0$ at $\nu^{a}=0$. So proper modulated rotating waves near $x_{0}$ correspond to nonlinear normal modes of the $\nu^{\phi}$-dependent $\dot{w}$-equation of $(3.23)$ at $\nu^{a}=0$. Note that $\mathcal{N}_{1}$ has dimension

$$
\operatorname{dim} \mathcal{N}_{1}=\operatorname{dim} \mathcal{M}-2 \operatorname{dim} \mathrm{SE}(2)=\operatorname{dim} \mathcal{M}-6 .
$$

By the Lyapounov Centre Theorem (see e.g. [18]) there are $d=\frac{\operatorname{dim} \mathcal{N}_{1}}{2}$ families of periodic orbits $w_{j}\left(\nu^{\phi}, s\right), j=1, \ldots, d$, of the $\dot{w}$-equation of $(3.23)$ such that $w_{j}\left(\nu^{\phi}, 0\right)=$ $w_{\mathrm{RW}}\left(\nu^{\phi}\right)$, with $w_{\mathrm{RW}}\left(\nu^{\phi}\right)$ from Proposition 4.2. Let $\mathrm{i} \omega_{j}\left(\nu^{\phi}\right)$ be the eigenvalue of $\mathbb{J}_{\mathcal{N}_{1}} \mathrm{D}_{w}^{2} h\left(\left(\nu^{\phi}, 0,0\right), w_{\mathrm{RW}}\left(\nu^{\phi}\right)\right)$ such that $\omega_{j}(0)=\omega_{j}$. Since $\partial_{s} w_{j}\left(\nu^{\phi}, 0\right)$ lies in the real eigenspace of $\mathbb{J}_{\mathcal{N}_{1}} \mathrm{D}_{w}^{2} h\left(\left(\nu^{\phi}, 0,0\right), w_{\mathrm{RW}}\left(\nu^{\phi}\right)\right)$ to the eigenvalue $\mathrm{i} \omega_{j}\left(\nu^{\phi}\right)$ (see e.g. [18]), and since $\mathrm{D}_{w} h\left(\left(\nu^{\phi}, 0,0\right), w_{\mathrm{RW}}\left(\nu^{\phi}\right)\right)=0$, the energy of the periodic orbits is

$$
h\left(\left(\nu^{\phi}, 0,0\right), w_{j}\left(\nu^{\phi}, s\right)\right)=H\left(x_{\mathrm{RW}}\left(\nu^{\phi}\right)\right)+\omega_{j}\left(\nu^{\phi}\right) s^{2}+O\left(s^{3}\right) .
$$

Therefore $s$ can be rescaled to achieve that the periodic orbit $w_{j}\left(\nu^{\phi}, s\right)$ has energy $H\left(x_{\mathrm{RW}}\left(\nu^{\phi}\right)\right) \pm$ $s^{2}$ depending on the sign of $\omega_{j}$, see (4.2). Then $x_{j}\left(\nu^{\phi}, s\right)=\left(\mathrm{id},\left(\nu^{\phi}, 0,0\right), w_{j}\left(\nu^{\phi}, s\right)\right)$ lies on an $\operatorname{RPO} \mathcal{P}_{j}\left(\nu^{\phi}, s\right)$ of (3.1). Its momentum is $\mathbf{J}\left(x_{j}\left(\nu^{\phi}, s\right)\right)=\left(\mu_{0}^{\phi}+\nu^{\phi}, 0,0\right)$, by (3.18). The drift symmetry $\gamma_{j}\left(\nu^{\phi}, s\right)=\left(\phi_{j}\left(\nu^{\phi}, s\right), a_{j}\left(\nu^{\phi}, s\right)\right)$ of the RPO at $x_{j}\left(\nu^{\phi}, s\right)$ satisfies $\phi_{j}(0,0)=2 \pi \omega_{0}^{\text {rot }} / \omega_{j}$, and so $\phi_{j}(0,0) \neq 0 \bmod 2 \pi$ if $\omega_{0}^{\text {rot }} / \omega_{j} \notin \mathbb{Z}$. In this case $\phi_{j}\left(\nu^{\phi}, s\right) \neq 0 \bmod 2 \pi$ for $\left(\nu^{\phi}, s\right) \approx 0$, and the RPOs $\mathcal{P}_{j}\left(\nu^{\phi}, s\right)$ are indeed proper modulated rotating waves for $\left(\nu^{\phi}, s\right) \approx 0$.

Example 4.7 Let us now study a Hamiltonian analogue of the meandering transition for point vortices with vanishing total circulation $\mathcal{K}=0$. In this case the momentum map of the point vortex system (3.3) is $\mathrm{Ad}^{*}$-equivariant, cf. Remark 3.4. Let us start with a configuration of rigidly rotating point vortices. Such a configuration is a rotating wave of (3.3). Synge [29] 


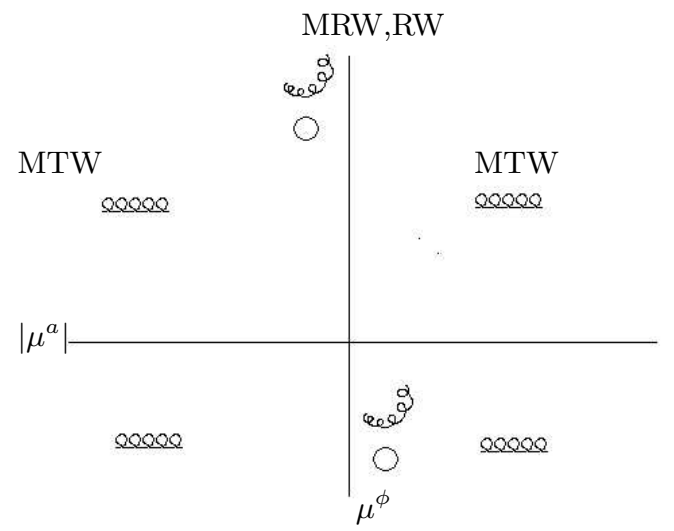

Figure 6: Bifurcation diagram for the Hamiltonian meandering transition of point vortex dynamics in the case of vanishing circulation. RW: rotating waves, MRW: modulated rotating waves, MTW: modulated travelling waves.

and later Aref [1] showed the existence of rotating waves of 3 vortices with vanishing total circulation. Patrick [25] constructed rotating wave solutions with vanishing total circulation for $N \geq 3$ vortices. Let us assume that the rotating wave is nondegenerate and that the nonresonance condition (4.1) is satisfied (this assumption is trivially satisfied for 3 vortices, since then $\mathcal{N}_{1}=\{0\}$ ). Then it persists as translating and precessing configuration, i.e. as modulated travelling wave, to non-zero linear momentum. Moreover if the rotating wave is elliptic and non-resonant then there are $\left(\frac{1}{2} \operatorname{dim} \mathcal{M}-3\right)=(N-3)$ different 2-parameter families of rotating and precessing configurations of vortices nearby, which are modulated rotating waves (Patrick [25] shows stability of the rotating waves of 4 vortices with vanishing total circulation, which he constructed. This implies that these rotating waves are elliptic, and, since $\mathcal{N}_{1}$ is 2 -dimensional, they are also nonresonant).

Figure 6 shows the bifurcation diagram of the Hamiltonian meandering transition for point vortex dynamics in the case of an $\mathrm{Ad}^{*}$-equivariant momentum map (for momentum maps with cocycle see Section 4.4). This diagram should be compared with the corresponding bifurcation diagram of the dissipative meandering transition, Figure 4.

Note that, in contrast to the meandering/drifting transition in dissipative systems, here modulated travelling waves are the typical scenario as momentum is varied. Modulated rotating waves only occur for zero-linear momentum and so are a codimension two phenomenon in the three parameters angular and linear momentum.

Example 4.8 Another example where a Hamiltonian meandering transition occurs is the Kirchhoff model of an underwater vehicle, see $[13,15]$. In this case the configuration space is the Euclidean group $\mathrm{SE}(3)=\mathrm{SO}(3) \ltimes \mathbb{R}^{3}$ of three-dimensional space modeling the angle and position of the underwater vehicle, and the phase space $\mathcal{M}=\mathcal{T}^{*} \mathrm{SE}(3)$ is 12-dimensional. In the case of non-coincident centres of gravity and buoyancy the symmetry group is

$$
\Gamma=\mathrm{SO}_{3}(2) \ltimes \mathbb{R}^{3}=\mathrm{SE}(2) \times \mathbb{R}_{3} .
$$

Here $\mathrm{SO}_{3}(2)$ denotes the group of rotations around the axis of gravity, which is chosen as the third coordinate axis (i.e., as the $e_{3}$-axis), and $\mathbb{R}_{3}$ is the group of translations along the $e_{3}$-axis. Near a vertically falling and spinning relative equilibrium the dynamics is given by the slice equations near a rotating wave of a Euclidean equivariant system (3.23), but now there is an additional equation

$$
\dot{\nu}_{3}^{a} \equiv 0
$$

in the slice and a corresponding equation

$$
\dot{a}_{3}=\mathrm{D}_{\nu_{3}^{a}} h(\nu, w)
$$


for the group dynamics, where $\nu=\left(\nu^{\phi}, \nu^{a}\right), \nu^{a}=\left(\nu_{1}^{a}, \nu_{2}^{a}, \nu_{3}^{a}\right)$. Assume that this relative equilibrium is nondegenerate and satisfies the non-resonance condition (4.1). Then it persists as translating RPO with non-vertical linear drift to horizontal linear momentum. Let the relative equilibrium be elliptic and non-resonant (from [15, Section 4.4.2] it follows that these conditions are satisfied at least for an open range of parameters). Then there are two 3-parameter families of RPOs, parametrized by $\left(\nu^{\phi}, \nu_{3}^{a}, s\right)$, which fall, rotate and precess.

\subsection{Hamiltonian meandering transition for momentum maps with co- cycle}

In this section the analogue of the meandering transition of dissipative systems is considered for Hamiltonian systems where the momentum map has a non-vanishing cocycle of the form that occurs in point vortex dynamics. The limiting behaviour for a vanishing cocycle is studied, and in this way the meandering transition for momentum maps with cocycle is related to the results of Sections 4.1-4.3 on the meandering transition for Hamiltonian systems with $\mathrm{Ad}^{*}$-equivariant momentum map.

Let us consider a parameter dependent SE(2)-symmetric Hamiltonian system (3.22). Assume that the symplectic form $\Omega(\mathcal{K})$, the Hamiltonian $H(\cdot, \mathcal{K})$ and the momentum map $\mathbf{J}(\cdot, \mathcal{K}): \mathcal{M} \rightarrow$ $\mathrm{se}(2)^{*}$ depend smoothly on a parameter $\mathcal{K}$. Moreover assume that the momentum map is $\mathrm{Ad}^{*}-$ equivariant for $\mathcal{K}=0$ and has the cocycle (3.11) for $\mathcal{K} \neq 0$, see (3.10), (3.12). An example of such a momentum map is the momentum map (3.4) for the point vortex dynamics (3.3).

As in the analysis of the Hamiltonian meandering transition for $\mathrm{Ad}^{*}$-equivariant momentum maps, see Section 4.1, first the persistence of rotating waves to nearby momentum values is studied. Moreover the behaviour of the rotating waves in the limit of vanishing cocycle is analyzed in the following theorem:

Theorem 4.9 Consider a Hamiltonian system with $\mathrm{SE}(2)$-symmetry for which the momentum map $\mathbf{J}(\cdot, \mathcal{K})$ has a non-vanishing cocycle satisfying (3.11), (3.12). Then:

a) For $\mathcal{K} \neq 0$ all relative equilibria are rotating waves. Any nondegenerate rotating wave $\mathrm{SE}(2) x_{0}$ persists to all nearby momentum values as a one-parameter family $\mathrm{SE}(2) x_{\mathrm{RW}}(\nu)$, $\nu \in\left(\mathbf{g}_{\mu_{0}}^{K}\right)^{*}, x_{\mathrm{RW}}(0)=x_{0}$.

b) The centre of rotation $c(\mathcal{K})$ of any smooth family of rotating waves $\mathrm{SE}(2) x_{\mathrm{RW}}(\mathcal{K}), \mathcal{K} \neq 0$, $\mathcal{K} \approx 0$, with fixed linear momentum $\mu^{a} \neq 0$ and rotation frequency $\omega^{\text {rot }}(\mathcal{K})$, such that $\lim _{\mathcal{K} \rightarrow 0} \omega^{\text {rot }}(\mathcal{K})=\omega_{0}^{\text {rot }} \neq 0$, tends to infinity, as $\mathcal{K} \rightarrow 0$, according to

$$
\|c(\mathcal{K})\|=\frac{\left\|\mu^{a}\right\|}{\mathcal{K}}
$$

c) Assume that there is a nondegenerate rotating wave $\mathrm{SE}(2) x_{0}$ at $\mathcal{K}=0$ with momentum $\mu_{0}=\left(\mu_{0}^{\phi}, 0\right)$ and rotation frequency $\omega_{0}^{\text {rot }} \neq 0$. Then this rotating wave can be continued to a rotating wave $\mathrm{SE}(2) x_{\mathrm{RW}}\left(\nu^{\phi}, \mathcal{K}\right)$ for small $\mathcal{K} \neq 0, \nu^{\phi} \neq 0$, such that $\mathbf{J}^{\phi}\left(x_{\mathrm{RW}}\left(\nu^{\phi}, \mathcal{K}\right), \mathcal{K}\right)=$ $\mu_{0}^{\phi}+\nu^{\phi}$ and $\mathbf{J}^{a}\left(x_{\mathrm{RW}}\left(\nu^{\phi}, \mathcal{K}\right), \mathcal{K}\right)=0$.

Part b) of this proposition implies that for $\mathcal{K}=0$ rotating waves only exist for vanishing linear momentum, see Proposition 4.2.

\section{Proof of Theorem 4.9.}

a) If the momentum map for a Hamiltonian system with $\mathrm{SE}(2)$-symmetry has a non-vanishing cocycle then $\Gamma_{\mu}^{\kappa} \simeq \mathrm{SO}(2)$ for all $\mu \in \operatorname{se}(2)^{*}$, as shown in Remark 3.4. Hence $\mathbf{g}_{\mu}^{K} \simeq \operatorname{so}(2)$ for all $\mu \in \operatorname{se}(2)^{*}$, and by (3.16) all relative equilibria are rotating waves. Moreover in Example 3.9 it was shown that $\dot{\nu} \equiv 0$ in $(3.21), \nu \in\left(\mathbf{g}_{\mu}^{K}\right)^{*}$. For a nondegenerate rotating wave $\mathrm{SE}(2) x_{0}$ the matrix $\mathrm{D}_{w}^{2} h(0)$ is invertible. Therefore there is a path $w_{\mathrm{RW}}(\nu)$ of equilibria of the $\dot{w}$-equation near $w_{\mathrm{RW}}(0)=0$. These equilibria correspond to rotating waves $\mathrm{SE}(2) x_{\mathrm{RW}}(\nu), x_{\mathrm{RW}}(\nu) \simeq\left(\mathrm{id}, \nu, w_{\mathrm{RW}}(\nu)\right)$, of (3.1) for all nearby momenta $\mathrm{SE}(2) \mathbf{J}\left(x_{\mathrm{RW}}(\nu), \mathcal{K}\right)=\mathrm{SE}(2)\left(\mu_{0}+\nu\right)$, where $\mu_{0}=\mathbf{J}\left(x_{0}, \mathcal{K}\right)$. 
b) Let $\mathrm{SE}(2) x_{\mathrm{RW}}(\mathcal{K})$ be a rotating wave with momentum $\mu$ and drift velocity $\xi=\xi(\mathcal{K})=$ $\left(\xi^{\phi}, \xi^{a}\right)(\mathcal{K})$. Differentiating $(3.15)$ and identifying $\mathbb{R}^{2}$ with $\mathbb{C}$ and so $(2)$ with $\mathbb{R}$ one obtains that

$$
\left(\xi^{\phi}, \xi^{a}\right) \in \mathbf{g}_{\mu}^{K} \Leftrightarrow \mathcal{K} \xi^{a}=\xi^{\phi} \mu^{a} .
$$

Let $(\phi, a) x=e^{\mathrm{i} \phi} x+a, x \in \mathbb{R}^{2} \simeq \mathbb{C}$. Then $x(t)=\exp \left(t\left(\xi^{\phi}, \xi^{a}\right)\right) x_{0}$ satisfies the differential equation $\dot{x}(t)=\mathrm{i} \xi^{\phi} x(t)+\xi^{a}$. Solving this and setting $t=1$ one gets

$$
\exp \left(\xi^{\phi}, \xi^{a}\right) x_{0}=e^{\mathrm{i} \xi^{\phi}} x_{0}+\frac{1}{\mathrm{i} \xi^{\phi}}\left(e^{\mathrm{i} \xi^{\phi}}-1\right) \xi^{a} .
$$

Therefore $\exp \left(\xi^{\phi}, \xi^{a}\right)$ is a rotation with centre

$$
c=\mathrm{i} \frac{\xi^{a}}{\xi^{\phi}}=\mathrm{i} \frac{\mu^{a}}{\mathcal{K}}=\frac{1}{\mathcal{K}}\left(-\mu_{2}^{a}, \mu_{1}^{a}\right)^{T} .
$$

This proves $\mathrm{b}$ ).

c) Let us only reduce by the $\mathrm{SO}(2) \times\{0\}$-symmetry. Then the system

$$
\dot{\tilde{\nu}}=0, \quad \dot{\tilde{w}}=\mathbb{J}_{\widetilde{\mathcal{N}}_{1}} \mathrm{D}_{\tilde{w}} \tilde{h}(\tilde{\nu}, \tilde{w}, \mathcal{K})
$$

is obtained on the slice $\tilde{\mathcal{N}}$ transverse to the $\mathrm{SO}(2)$-orbit $\mathrm{SO}(2) x_{0}$ at $x_{0}$, with $\tilde{\nu}=\nu^{\phi}$. By (3.8), (3.9) the matrix $\operatorname{ad}_{\xi_{0}}=-\operatorname{ad}_{\xi_{0}}^{*}$ has simple eigenvalues 0 and $\pm \mathrm{i} \omega_{0}^{\text {rot }}$. By (2.4) and (3.20), at $\mathcal{K}=0$ the linearization of the slice equation $\mathrm{D} f_{\tilde{\mathcal{N}}}(0,0)$ has one simple eigenvalue 0 corresponding to the equation $\dot{\tilde{\nu}}=0$. Therefore $\mathrm{D}_{\tilde{w}}^{2} \tilde{h}(0,0,0)$ is invertible and so the rotating wave $\mathrm{SO}(2) x_{0}$ is nondegenerate. By Remark 3.7 the $\dot{\tilde{w}}$-equation is smooth in $\mathcal{K}$. So there is a smooth two-parameter family $\tilde{w}_{\mathrm{RW}}(\tilde{\nu}, \mathcal{K})$ of equilibria of the $\dot{\tilde{w}}$-equation. This gives a family $\mathrm{SE}(2) x_{\mathrm{RW}}\left(\nu^{\phi}, \mathcal{K}\right), x_{\mathrm{RW}}\left(\nu^{\phi}, \mathcal{K}\right) \simeq\left(\mathrm{id}, \nu^{\phi}, \tilde{w}_{\mathrm{RW}}\left(\nu^{\phi}, \mathcal{K}\right)\right)$, of rotating waves of (3.1). By (3.18) their angular momentum is $\mathbf{J}^{\phi}\left(x_{\mathrm{RW}}\left(\nu^{\phi}, \mathcal{K}\right), \mathcal{K}\right)=\mu_{0}^{\phi}+\nu^{\phi}$. Since only a reduction by the $\mathrm{SO}(2)$-symmetry has been carried out, the rotating waves through $x_{\mathrm{RW}}\left(\nu^{\phi}, \mathcal{K}\right)$ have drift velocities $\xi_{\mathrm{RW}}\left(\nu^{\phi}, \mathcal{K}\right)$ with $\xi_{\mathrm{RW}}^{a}\left(\nu^{\phi}, \mathcal{K}\right)=0$. Moreover $\xi_{\mathrm{RW}}^{\phi}\left(\nu^{\phi}, \mathcal{K}\right) \approx$ $\omega_{0}^{\text {rot }} \neq 0$ for $\mathcal{K} \approx 0$. Therefore $(4.3)$ implies that $\mathbf{J}^{a}\left(x_{\mathrm{RW}}\left(\nu^{\phi}, \mathcal{K}\right), \mathcal{K}\right)=0$.

The next proposition shows that in the case of a momentum map with cocycle (3.11) all relative periodic orbits are modulated rotating waves:

Proposition 4.10 Consider a Hamiltonian system with SE(2)-symmetry for which the momentum map $\mathbf{J}(\cdot, \mathcal{K})$ has a non-vanishing cocycle satisfying (3.11), (3.12). Then:

a) For $\mathcal{K} \neq 0$ all relative periodic orbits are modulated rotating waves.

b) Any smooth family $\mathcal{P}(\mathcal{K})$ of proper modulated rotating waves of (3.1), i.e., of RPOs with drift symmetry $\gamma(\mathcal{K})=(\phi(\mathcal{K}), a(\mathcal{K}))$, where $\phi(\mathcal{K}) \neq 0 \bmod 2 \pi$ for all $\mathcal{K} \neq 0, \mathcal{K} \approx 0$, with fixed linear momentum $\mu^{a} \neq 0$ has a centre of rotation $c(\mathcal{K})$ diverging to $\infty$ for $\mathcal{K} \rightarrow 0$ according to $\|c(\mathcal{K})\|=\frac{\left\|\mu^{a}\right\|}{\mathcal{K}}$.

\section{Proof.}

a) Similarly as in the proof of Theorem 4.9 a), this follows from the fact that $\Gamma_{\mu}^{\kappa} \simeq \mathrm{SO}(2)$ for all $\mu \in \operatorname{se}(2)^{*}$, as shown in Remark 3.4. This, together with (3.17), implies that all relative periodic orbits are modulated rotating waves.

b) This statement is proved similarly as Theorem $4.9 \mathrm{~b})$. By $(3.15)$, the centre of rotation $c(\mathcal{K})=R_{\phi(\mathcal{K})} c(\mathcal{K})+a(\mathcal{K})$ of the drift symmetry $\gamma(\mathcal{K})=(\phi(\mathcal{K}), a(\mathcal{K})), \phi(\mathcal{K}) \neq 0 \bmod 2 \pi$, of the RPO is given by (4.4), and so $c(\mathcal{K}) \rightarrow \infty$ as $\mathcal{K} \rightarrow 0$ for $\mu^{a} \neq 0$. 
In the next theorem the transition from rotating waves to modulated rotating waves and modulated travelling waves is studied, in the limit $\mathcal{K} \rightarrow 0$ :

Theorem 4.11 Let $\mathrm{SE}(2) x_{\mathrm{RW}}(\mathcal{K})$ be a nondegenerate rotating wave of a Hamiltonian system (3.1) which has a momentum map $\mathbf{J}(\cdot, \mathcal{K})$ with cocycle satisfying (3.10), (3.11). Fix the momentum $\mu_{0}=\mathbf{J}\left(x_{\mathrm{RW}}(\mathcal{K}), \mathcal{K}\right)$ of $x_{\mathrm{RW}}(\mathcal{K})$ independent of $\mathcal{K}$. Then the following holds true.

a) Fix $\mathcal{K} \neq 0$. Assume that the rotating wave $\mathrm{SE}(2) x_{\mathrm{RW}}(\mathcal{K})$ is elliptic and nonresonant in the sense of Definition 4.4 , with normal frequencies $\omega_{j}, j=1, \ldots, \frac{1}{2} \operatorname{dim} \mathcal{M}-2$. Then there are $\left(\frac{1}{2} \operatorname{dim} \mathcal{M}-2\right)$ many two-dimensional families $\mathcal{P}_{j}(\nu, s, \mathcal{K})$ of modulated rotating waves such that there are functions $x_{j}(\nu, s, \mathcal{K}) \in \mathcal{P}_{j}(\nu, s, \mathcal{K})$ which are smooth in $s \geq 0, \mathcal{K}$ and $\nu \in\left(\mathrm{g}_{\mu_{0}}^{K}\right)^{*} \simeq \mathrm{so}(2)^{*}$ with $x_{j}(\nu, 0, \mathcal{K})=x_{\mathrm{RW}}(\nu, \mathcal{K})$. Here $x_{\mathrm{RW}}(\nu, \mathcal{K})$ lies on a rotating wave with momentum $\mu_{0}+\nu$. The modulated rotating wave through $x_{j}(\nu, s, \mathcal{K})$ has relative period $T_{j}(\nu, s, \mathcal{K})$ with $T_{j}(0,0, \mathcal{K})=2 \pi /\left|\omega_{j}\right|$, its momentum is $\mathbf{J}\left(x_{j}(\nu, s, \mathcal{K}), \mathcal{K}\right)=\mu_{0}+\nu$, and its energy is $H\left(x_{j}(\nu, s, \mathcal{K}), \mathcal{K}\right)=H\left(x_{\mathrm{RW}}(\nu, \mathcal{K}), \mathcal{K}\right) \pm s^{2}$ (depending on the Krein signature of $\left.\omega_{j}\right), j=1, \ldots, \frac{1}{2} \operatorname{dim} \mathcal{M}-2$.

b) Assume that there is a rotating wave $\mathrm{SE}(2) x_{0}$ at $\mathcal{K}=0$ with rotation frequency $\omega_{0}^{\mathrm{rot}}$ which is elliptic and nonresonant in the sense of Definition 4.4 , and that $\omega_{0}^{\text {rot }} / \omega_{j} \notin \mathbb{Z}$ for all eigenvalues $\mathrm{i} \omega_{j}$ of $\mathbb{J}_{\mathcal{N}_{1}} \mathrm{D}_{w}^{2} h(0,0,0)$. Then the $\left(\frac{1}{2} \operatorname{dim} \mathcal{M}-3\right)$ families $\mathcal{P}_{j}\left(\nu^{\phi}, s\right)$ of modulated rotating waves near $\mathrm{SE}(2) x_{0}$ from Proposition 4.6 at $\mathcal{K}=0$ can be continued to small $\mathcal{K} \neq 0$ and correspond to families $\mathcal{P}_{j}(\nu, s, \mathcal{K})$ from part a) with $\nu=\nu^{\phi}$.

c) Assume that the rotating wave $\operatorname{SE}(2) x_{0}$ at $\mathcal{K}=0$ is elliptic and nonresonant and that $\omega_{j} / \omega_{0}^{\text {rot }} \notin \mathbb{Z}$ for all eigenvalues $\mathrm{i} \omega_{j}$ of $\mathbb{J}_{\mathcal{N}_{1}} \mathrm{D}_{w}^{2} h(0,0,0)$. Then for $\mathcal{K} \neq 0$, $\mathcal{K} \approx 0$, one of the families $\mathcal{P}(\nu, s, \mathcal{K})$ from a) corresponds to an eigenvalue $i \omega(\mathcal{K})$ of $\mathbb{J}_{\mathcal{N}_{1}(\mathcal{K})} \mathrm{D}_{w}^{2} h\left(0, w_{\mathrm{RW}}(0, \mathcal{K}), \mathcal{K}\right)$ which depends smoothly on $\mathcal{K}$ such that $\omega(0)=\omega_{0}^{\text {rot }}$. Here $\omega_{0}^{\mathrm{rot}}$ is the rotation frequency of the rotating wave $\mathrm{SE}(2) x_{0}$ at $\mathcal{K}=0, \mathcal{N}_{1}(\mathcal{K})$ is the symplectic normal space at the rotating wave through $x_{\mathrm{RW}}(0, \mathcal{K}) \simeq\left(\mathrm{id}, 0, w_{\mathrm{RW}}(0, \mathcal{K})\right) \in$ $\Gamma \times \mathcal{N}_{0}(\mathcal{K}) \oplus \mathcal{N}_{1}(\mathcal{K}), \mathcal{N}_{0}(\mathcal{K}) \simeq\left(\mathbf{g}_{\mu_{0}}^{K}\right)^{*}$, and $h(\nu, w, \mathcal{K})$ is the Hamiltonian in symmetry adapted coordinates at $x_{\mathrm{RW}}(0, \mathcal{K})$ for the cocycle parameter $\mathcal{K}$. As $\mathcal{K} \rightarrow 0$, this family converges to the family of modulated travelling waves from Theorem 4.3.

\section{Proof.}

a) Fix $\mathcal{K} \neq 0$. Then, as shown in Example $3.9, \mathcal{N}_{0}=\mathcal{N}_{0}(\mathcal{K})$ is one-dimensional, and so $\mathcal{N}_{1}=\mathcal{N}_{1}(\mathcal{K})$ has dimension $\operatorname{dim} \mathcal{M}-4$. By the Lyapounov Centre Theorem (see e.g. [18]) applied to the $\nu$-dependent $\dot{w}$-equation on $\mathcal{N}_{1}=\mathcal{N}_{1}(\mathcal{K})$, there are $\frac{1}{2} \operatorname{dim} \mathcal{N}_{1}=(\operatorname{dim} \mathcal{M}-$ 4)/2 many families of nonlinear normal modes through $w_{j}(\nu, s, \mathcal{K})$. These give families of relative periodic orbits $\mathcal{P}_{j}(\nu, s, \mathcal{K})$ of $(3.22)$ through $x_{j}(\nu, s, \mathcal{K}) \simeq\left(\mathrm{id}, \nu, w_{j}(\nu, s, \mathcal{K})\right)$ with momentum $\mathbf{J}\left(x_{j}(\nu, s, \mathcal{K})\right)=\mu_{0}+\nu$, see (3.18). The statement about the energy of the RPOs is proved as in Proposition 4.6. It was shown in Remark 3.4 that $\Gamma_{\mu}^{\kappa} \simeq \mathrm{SO}(2)$ for all $\mu \in \operatorname{se}(2)^{*}$. Hence (3.17) implies that all these RPOs are modulated rotating waves. This proves part a).

b) Let us reduce by $\mathrm{SO}(2)$-symmetry only. The dynamics on the slice $\tilde{\mathcal{N}}(\mathcal{K})$ is then $\dot{\tilde{\nu}} \equiv 0$, $\tilde{\nu}=\nu^{\phi} \in \operatorname{so}(2)^{*}$, and $\dot{\tilde{w}}=\mathbb{J}_{\widetilde{\mathcal{N}}_{1}(\mathcal{K})} \mathrm{D}_{\tilde{w}} \tilde{h}(\tilde{\nu}, \tilde{w}, \mathcal{K})$. Since $\operatorname{ad}_{\xi_{0}}=-\operatorname{ad}_{\xi_{0}}^{*}$ has simple eigenvalues $0, \pm \mathrm{i} \omega_{0}^{\text {rot }}$, c.f. (3.8), (3.9), (2.4) and (3.20) imply that the equilibrium $\tilde{w}=0$ of the $\dot{\tilde{w}}$-equation at $\tilde{\nu}=0, \mathcal{K}=0$, is elliptic and that its linearization has, in addition to the eigenvalues $\pm \mathrm{i} \omega_{j}, j=1, \ldots, \frac{1}{2} \operatorname{dim} \mathcal{N}_{1}$, double eigenvalues $\pm \mathrm{i} \omega_{0}^{\text {rot }}$. Applying the Lyapounov Centre Theorem to the $\dot{\tilde{w}}$-equation, relative normal modes $\tilde{w}_{j}(\tilde{\nu}, s, \mathcal{K})$ are obtained for all nonresonant normal frequencies, i.e., for all imaginary eigenvalues $\pm \mathrm{i} \omega_{j}$ with eigenvectors in the original symplectic normal space $\mathcal{N}_{1}$ for the full SE(2)-group action. Since by Remark 3.7 the $\dot{\tilde{w}}$-equation is smooth in $\mathcal{K}$, this gives $\frac{1}{2} \operatorname{dim} \mathcal{N}_{1}=(\operatorname{dim} \mathcal{M}-6) / 2$ smooth families $\mathcal{P}_{j}\left(\nu^{\phi}, s, \mathcal{K}\right)$ of RPOs through $x_{j}\left(\nu^{\phi}, s, \mathcal{K}\right) \simeq\left(\mathrm{id}, \tilde{\nu}, \tilde{w}_{j}\left(\nu^{\phi}, s, \mathcal{K}\right)\right), j=1, \ldots \frac{1}{2} \operatorname{dim} \mathcal{N}_{1}$, with momentum $\mathbf{J}\left(x_{j}\left(\nu^{\phi}, s, \mathcal{K}\right)\right)=\left(\mu_{0}^{\phi}+\nu^{\phi}, 0\right)$, see (3.18). These RPOs are modulated rotating waves, since the system was only reduced by $\mathrm{SO}(2)$-symmetry. 
c) Let $\mathcal{K}=0$. Then (3.20) and (3.9) imply that the linearization of the $\dot{\nu}$-equation has eigenvalues $\pm \mathrm{i} \omega_{0}^{\mathrm{rot}}$ and 0 , where the real eigenspace of $\pm \mathrm{i} \omega_{0}^{\mathrm{rot}}$ is given by $\left\{\nu=\left(0, \nu^{a}\right), \nu^{a} \in\right.$ $\left.\mathbb{R}^{2}\right\} \subseteq \operatorname{se}(2)^{*}$. Compared to the slice equation (3.23) near rotating waves of momentum maps without cocycle, for $\mathcal{K} \neq 0$ the $\mathcal{N}_{0}(\mathcal{K})$ component of the slice $\mathcal{N}(\mathcal{K})$ is only 1dimensional instead of 3-dimensional. Since $\mu_{0}^{a}=\mathbf{J}^{a}\left(x_{0}, 0\right)=0$ and $\mu_{0}=\mathbf{J}\left(x_{\mathrm{RW}}(\mathcal{K}), \mathcal{K}\right)$ is fixed, Remark 3.9 implies that $\nu=\nu^{\phi}$ for $\mathcal{K} \neq 0$ and that then $\dot{\nu} \equiv 0$. For $\mathcal{K} \neq 0$ the eigenvalue $\pm \mathrm{i} \omega_{0}^{\text {rot }}$ of $\mathrm{D} f_{\mathcal{N}}(0)$ perturbs into eigenvalues $\pm \mathrm{i} \omega(\mathcal{K})$ of $\mathrm{D} f_{\mathcal{N}(\mathcal{K})}\left(v_{\mathrm{RW}}(\mathcal{K})\right)$. Here $v_{\mathrm{RW}}(\mathcal{K})$ is the equilibrium of the slice equation at momentum $\mu_{0}$ and cocycle parameter $\mathcal{K}$ corresponding to the relative equilibrium $x_{\mathrm{RW}}\left(\nu^{\phi}, \mathcal{K}\right)$ at $\nu^{\phi}=0$ from Theorem $\left.4.9 \mathrm{c}\right)$. Since $\mathrm{D} f_{\mathcal{N}(\mathcal{K})}\left(v_{\mathrm{RW}}(\mathcal{K})\right)$ vanishes when restricted to $\mathcal{N}_{0}(\mathcal{K})$, it follows that $\pm \mathrm{i} \omega(\mathcal{K})$ are eigenvalues of $\mathrm{D} f_{\mathcal{N}_{1}(\mathcal{K})}\left(v_{\mathrm{RW}}(\mathcal{K})\right)$. By the Lyapounov Centre Theorem and due to the nonresonance condition $\omega_{j} / \omega_{0}^{\text {rot }} \notin \mathbb{Z}$, this gives one more family of nonlinear relative normal modes for $\mathcal{K} \neq 0, \mathcal{K} \approx 0$. It will now be shown that this additional family of RPOs converges to the family $\mathcal{P}\left(\nu^{\phi}, r^{a}\right)$ of modulated traveling waves from Theorem 4.3 as $\mathcal{K} \rightarrow 0$.

The proof of Theorem 4.3 can be extended to the case $\mathcal{K} \approx 0, \mathcal{K} \neq 0$. It is convenient to work in the slice coordinates $(\nu, w) \in \mathcal{N}=\mathcal{N}_{0} \oplus \mathcal{N}_{1}$ at $\mathcal{K}=0$, even when perturbations to cocycle parameters $\mathcal{K} \neq 0$ are considered. For any $\mathcal{K} \approx 0$ the dynamics on the slice $\mathcal{N}$ does not depend on $\gamma$ and the energy $h(\nu, w, \mathcal{K})$ is still a conserved quantity. But note that the Poisson structure on $\mathcal{N}$ changes, and in particular $\left|\nu^{a}\right|^{2}$ is not a conserved quantity any more. By (3.9), for $\mathcal{K}=0$ the momentum group orbits $\mathrm{SE}(2) \mu$ are cylinders around the $\mu^{\phi}$-axis. For $\mathcal{K} \neq 0$, due to (3.10) and (3.11), they are paraboloids, centered along the $\mu^{\phi}$-axis. A Casimir, i.e., a function satisfying $C\left(\gamma \cdot{ }_{\kappa} \mu\right)=C(\mu)$, where $\gamma \cdot{ }_{\kappa} \mu$ is defined in (3.10), is given by $C\left(\mu^{\phi}, \mu^{a}\right)=\left\|\mu^{a}\right\|^{2}+2 \mathcal{K} \mu^{\phi}$. Let $\mathbf{J}^{a}(\nu, w, \mathcal{K})$ and $\mathbf{J}^{\phi}(\nu, w, \mathcal{K})$ be the linear and angular momentum on the slice $\mathcal{N}$. Since the momentum map depends smoothly on $\mathcal{K}$, these maps are smooth in all variables. Then

$$
C(\nu, w, \mathcal{K})=\left\|\mathbf{J}^{a}(\nu, w, \mathcal{K})\right\|^{2}+2 \mathcal{K} \mathbf{J}^{\phi}(\nu, w, \mathcal{K})
$$

is a conserved quantity for the slice equation. For $\mathcal{K}=0$ one has $\mathbf{J}^{a}(\nu, w, \mathcal{K}=0)=\nu^{a}$, $\mathbf{J}^{\phi}(\nu, w, \mathcal{K}=0)=\nu^{\phi}$. Therefore for $\mathcal{K} \approx 0$ the Casimir $C(\nu, w, \mathcal{K})$ is a small perturbation of $\left.C(\nu, w, \mathcal{K})\right|_{\mathcal{K}=0}=\left\|\nu^{a}\right\|^{2}$. The rotating waves through $x_{\mathrm{RW}}\left(\nu^{\phi}, \mathcal{K}\right)$ from Theorem 4.9 c) have energy $E_{\mathrm{RW}}\left(\nu^{\phi}, \mathcal{K}\right)=H\left(x_{\mathrm{RW}}\left(\nu^{\phi}, \mathcal{K}\right)\right)$ with $\frac{\partial E_{\mathrm{RW}}}{\partial \nu^{\phi}}(0,0)=\omega_{0}^{\text {rot }} \neq 0$. So they can be parametrized by $(E, \mathcal{K})$ instead of $\left(\nu^{\phi}, \mathcal{K}\right)$ for $E \approx E_{0}=H\left(x_{0}\right)$ and $\mathcal{K} \approx 0$. Let $x_{\mathrm{RW}}(E, \mathcal{K})=\left(\mathrm{id}, \nu_{\mathrm{RW}}(E, \mathcal{K}), w_{\mathrm{RW}}(E, \mathcal{K})\right)$ be the family of rotating waves near $x_{0} \simeq$ $(\mathrm{id}, 0,0) \in \Gamma \times \mathcal{N}$. Then $\nu_{\mathrm{RW}, 1}^{a}(E, 0)=0, \nu_{\mathrm{RW}, 2}^{a}(E, 0)=0$ by Proposition 4.2. Since the slice equation conserves energy, the energy level sets $\mathcal{N}_{E, \mathcal{K}}$ of the slice $\mathcal{N}$ at cocycle parameter $\mathcal{K}$ are flow-invariant. Moreover, as $\mathrm{D}_{\nu^{\phi}} h(0)=\omega_{0}^{\text {rot }}, \omega_{0}^{\text {rot }} \neq 0$, they can be parametrized by

$$
\mathcal{N}_{E, \mathcal{K}}=\{(\nu, w) \in \mathcal{N}, \quad h(\nu, w, \mathcal{K})=E\}=\left\{(\nu, w) \in \mathcal{N}, \quad \nu^{\phi}=\nu^{\phi}\left(E, \nu^{a}, w, \mathcal{K}\right)\right\}
$$

where $E \approx E_{0}=H\left(x_{0}\right), \nu^{a} \approx 0, \mathcal{K} \approx 0, w \approx 0$. As in the proof of Theorem 4.3,

$$
\begin{gathered}
\mathcal{S}_{E, \mathcal{K}}=\left\{(\nu, w) \in \mathcal{N}, \quad \nu_{2}^{a}=\nu_{\mathrm{RW}, 2}^{a}(E, \mathcal{K}), \quad \nu_{1}^{a}>\nu_{\mathrm{RW}, 1}^{a}(E, \mathcal{K}), \quad \nu^{\phi}=\nu^{\phi}\left(E, \nu^{a}, w, \mathcal{K}\right),\right. \\
\left.w \in \mathcal{N}_{1}, \quad w \approx 0, \quad \nu^{a} \approx 0\right\}
\end{gathered}
$$

is a Poincaré section in $\mathcal{N}_{E, \mathcal{K}}$ for $E \approx E_{0}, \mathcal{K} \approx 0$. Denote $s=\nu_{1}^{a}-\nu_{\mathrm{RW}, 1}^{a}(E, \mathcal{K})$. Let us now look for fixed points of the Poincaré map $\Pi(E, s, w, \mathcal{K})$ which maps $\mathcal{S}_{E, \mathcal{K}}$ to itself.

Decompose $\Pi(E, s, w, \mathcal{K})=\left(\Pi_{0}(E, s, w, \mathcal{K}), \Pi_{1}(E, s, w, \mathcal{K})\right)$, where $\Pi_{1}(E, s, w, \mathcal{K})$ is the $w$ component $\left(w \in \mathcal{N}_{1}\right)$ and $\Pi_{0}(E, s, w, \mathcal{K})$ the $s$-component of $\Pi$. Due to the nonresonance condition, $\Pi_{1}(E, s, w, \mathcal{K})=w$ can be solved for $\mathcal{K} \approx 0, s \approx 0, E \approx E_{0}=H\left(x_{0}\right)$, by the implicit function theorem to obtain $w(E, s, \mathcal{K})$. Inserting this into $\Pi_{0}$, one obtains one scalar fixed point equation $s=\hat{\Pi}(E, s, \mathcal{K})$. This equation is satisfied due to the existence of the Casimir (4.5): Let $\hat{s}=\hat{\Pi}(E, s, \mathcal{K})$. Inserting $w=w(E, s, \mathcal{K})$ into (4.5), a function $C(E, s, \mathcal{K})=s^{2}+O(\mathcal{K})$ is obtained. Any equilibrium $(\nu, w)$ of the slice equation on $\mathcal{N}$ satisfies

$$
\mathrm{D} C(\nu, w) \| \mathrm{D} h(\nu, w)
$$




$$
\mathrm{D}\left(\left.C\right|_{h=E}\right)=0,
$$

where $E=h(\nu, w)$. The equilibria corresponding to rotating waves of (3.1) are at $s=0$ and therefore $\mathrm{D}_{s} C(E, 0, \mathcal{K}) \equiv 0$. Moreover $\mathrm{D}_{s}^{2} C\left(E_{0}, 0,0\right)=2$, where $E_{0}=H\left(x_{0}\right)$. So $s \rightarrow C(E, s, \mathcal{K})$ is monotonically increasing for $s \geq 0, E \approx E_{0}, \mathcal{K} \approx 0$, and one can solve for $s(E, C, \mathcal{K})$. Hence $\hat{s}=s$, and a family $(\nu, w)(E, C, \mathcal{K})$ of periodic orbits of the slice equation is obtained. This gives a family $\mathcal{P}(E, C, \mathcal{K})$ of RPOs of $(3.22)$. Changing the parametrization of the RPOs $\mathcal{P}(E, C, \mathcal{K})$ from $(E, C)$ back to $\left(\nu^{\phi}, s\right)$, the notation of the theorem is recovered. Let $(\phi, a)\left(\nu^{\phi}, s, \mathcal{K}\right)$ by the drift symmetry of the RPO $\mathcal{P}\left(\nu^{\phi}, s, \mathcal{K}\right)$ at $x\left(\nu^{\phi}, s, \mathcal{K}\right) \simeq\left(\mathrm{id},(\nu, w)\left(\nu^{\phi}, s, \mathcal{K}\right)\right)$. Note that for $\mathcal{K}=0,(3.18)$ gives $s=r^{a}$ and $\mathbf{J}\left(x\left(\nu^{\phi}, r^{a}, 0\right)=\left(\mu_{0}^{\phi}+\nu^{\phi}, r^{a}, 0\right)\right.$. By (3.17) and (3.9) for $r^{a} \neq 0$ and $\mathcal{K}=0$ all RPOs are modulated traveling waves. Therefore $\phi\left(\nu^{\phi}, s, \mathcal{K}\right) \rightarrow 0$ as $\mathcal{K} \rightarrow 0$ and the RPOs $\mathcal{P}\left(\nu^{\phi}, s, \mathcal{K}\right)$ become modulated traveling waves in the limit of vanishing cocycle.

\section{$5 \quad$ Extensions to systems with other symmetry groups}

In this section the Hamiltonian analogue of the meandering transition is discussed for systems with spherical symmetry and for systems with the Euclidean symmetry group of threedimensional space. See Remarks 2.2 b) and c) for the corresponding dissipative case.

\subsection{Hamiltonian meandering transition with spherical symmetry}

In this section it is assumed that the Hamiltonian system (3.1) has spherical symmetry $\Gamma=$ $\mathrm{SO}(3)$. Then persistence of rotating waves to modulated rotating waves at nearby momentum values is studied. Thereby the analogue of Remark $2.2 \mathrm{~b}$ ) is studied in the Hamiltonian context. The results can be applied to rotating point vortices on the sphere, see e.g. [19].

For $\mu_{0}=0$ the momentum isotropy subalgebra is $\mathbf{g}_{\mu_{0}}=\mathrm{so}(3)$ and for $\mu_{0} \neq 0$ (the typical case) it is $\mathbf{g}_{\mu_{0}}=\mathrm{so}(2)$. Let us first consider the generic case of a rotating wave with momentum $\mu_{0} \neq 0$.

Theorem 5.1 Let $\mathrm{SO}(3) x_{0}$ be a nondegenerate rotating wave with non-vanishing momentum $\mu_{0}$ and drift velocity $\xi_{0}$. Align $x_{0}$ such that $\mu_{0}=\left(0,0, \mu_{0,3}\right), \xi_{0}=\left(0,0, \omega_{0}^{\text {rot }}\right)^{T}$. Then the following holds true:

a) The rotating wave $\mathrm{SO}(3) x_{0}$ persists to every nearby momentum. Moreover there is a onedimensional family of rotating waves $\mathrm{SO}(3) x_{\mathrm{RW}}(\nu), \nu \approx 0$, such that $x_{\mathrm{RW}}(\nu)$ is smooth and $x_{\mathrm{RW}}(0)=x_{0}$. The rotating wave through $x_{\mathrm{RW}}(\nu)$ has drift velocity $\xi_{\mathrm{RW}}(\nu) \| e_{3}$, with $\xi_{\mathrm{RW}}(0)=\xi_{0}$, and momentum $\mathbf{J}\left(x_{\mathrm{RW}}(\nu)\right)=\left(0,0, \mu_{0,3}+\nu\right)$.

b) Let the rotating wave $\mathrm{SO}(3) x_{0}$ be elliptic and nonresonant in the sense of Definition 4.4 and denote its normal frequencies by $\omega_{j}$. Then there are $\left(\frac{1}{2} \operatorname{dim} \mathcal{M}-2\right)$ two-dimensional families $\mathcal{P}_{j}(\nu, s), j=1, \ldots \frac{1}{2} \operatorname{dim} \mathcal{M}-2, \nu \approx 0, s \geq 0$, of modulated rotating waves nearby such that there are smooth functions $x_{j}(\nu, s) \in \mathcal{P}_{j}(\nu, s)$ with $x_{j}(\nu, 0)=x_{\mathrm{RW}}(\nu)$ (where $x_{\mathrm{RW}}(\nu)$ is from a)). These modulated rotating waves have energy $H\left(x_{j}(\nu, s)\right)=H\left(x_{\mathrm{RW}}(\nu)\right) \pm s^{2}$ (depending on the Krein signature of $\left.\omega_{j}\right)$, momentum $\mathbf{J}\left(x_{j}(\nu, s)\right)=\left(0,0, \mu_{0,3}+\nu\right)$, relative period $T_{j}(\nu, s)$, such that $T_{j}(0,0)=\frac{2 \pi}{\left|\omega_{j}\right|}$, and average drift velocity $\xi_{j}(\nu, s) \| e_{3}$ at $x_{j}(\nu, s)$, with $\xi_{j}(0,0)=\xi_{0}$.

So resonance drift can not occur near rotating waves of $\mathrm{SO}(3)$-symmetric Hamiltonian systems with non-vanishing angular momentum.

Proof of Theorem 5.1 If $\mu_{0} \neq 0$ then $\mathcal{N}_{0} \simeq \mathbf{g}_{\mu_{0}}^{*} \simeq \mathrm{so}_{3}(2)^{*}$ is one-dimensional. Here $\mathrm{so}_{3}(2)$ corresponds to infinitesimal rotations around the $e_{3}$-axis. So the $\dot{\nu}$-equation of (3.19) just becomes $\dot{\nu}=0$. 
a) A nondegenerate rotating wave $\mathrm{SO}(3) x_{0}$ persists as equilibrium $w_{\mathrm{RW}}(\nu)$ of the $\dot{w}$-equation for $\nu \approx 0$. This gives a rotating wave of $(3.1)$ through $x_{\mathrm{RW}}(\nu) \simeq\left(\mathrm{id}, \nu, w_{\mathrm{RW}}(\nu)\right)$ with nonvanishing momentum $\mathbf{J}\left(x_{\mathrm{RW}}(\nu)\right)=\left(0,0, \mu_{0,3}+\nu\right), \nu \approx 0$, c.f. (3.18). Due to $\mathrm{SO}(3)$ equivariance the rotating wave persists to all nearby momenta.

b) By the Lyapounov Centre Theorem there are $\frac{1}{2} \operatorname{dim}\left(\mathcal{N}_{1}\right)$ families $w_{j}(\nu, s)$ of periodic orbits of the $\dot{w}$-equation, parametrized by $\nu$ and $s$. Here $\operatorname{dim} \mathcal{N}_{1}=\operatorname{dim} \mathcal{M}-4$. These give points $x_{j}(\nu, s)=\left(\mathrm{id}, \nu, w_{j}(\nu, s)\right)$ on modulated rotating waves $\mathcal{P}_{j}(\nu, s)$ with $x_{j}(\nu, 0)=x_{\mathrm{RW}}(\nu)$ and with momentum $\mathbf{J}\left(x_{j}(\nu, s)\right)=\mu_{0}+\nu=\left(0,0, \mu_{0,3}+\nu_{3}\right)$, see (3.18). Let $\mathrm{SO}_{3}(2)$ be the group of rotations around $e_{3}$ with Lie algebra $\operatorname{so}_{3}(2)$. Since $\mathbf{J}\left(x_{j}(\nu, s)\right) \neq 0$ and $\mathbf{J}\left(x_{j}(\nu, s)\right) \| e_{3}$, by $(3.17)$ the drift symmetry $R_{j}(\nu, s)$ of the RPO at $x_{j}(\nu, s)$ lies in $\mathrm{SO}_{2}(3)$, and so the average drift velocity $\xi_{j}(\nu, s)$ at $x_{j}(\nu, s)$ is in $\operatorname{so}_{3}(2)$.

Next let us consider the case that the rotating wave $\mathrm{SO}(3) x_{0}$ has zero angular momentum $\mu_{0}=0$. In this case resonance drift typically occurs as the following theorem shows:

Theorem 5.2 Consider a nondegenerate rotating wave $\mathrm{SO}(3) x_{0}$ with momentum $\mu_{0}=\mathbf{J}\left(x_{0}\right)=$ 0 and non-vanishing drift velocity $\xi_{0} \neq 0$. Choose $x_{0}$ such that $\xi_{0}=\left(0,0, \omega_{0}^{\mathrm{rot}}\right)^{T}$ where $\omega_{0}^{\mathrm{rot}}$ is the rotation frequency of the rotating wave at $x_{0}$. Then the following holds true:

a) There is a one-parameter family $\mathrm{SO}(3) x_{\mathrm{RW}}\left(\nu_{3}\right)$ of rotating waves nearby, $\nu_{3} \approx 0$, with momentum $\mathbf{J}\left(x_{\mathrm{RW}}\left(\nu_{3}\right)\right)=\left(0,0, \nu_{3}\right)$ and drift velocity $\xi_{\mathrm{RW}}\left(\nu_{3}\right) \| e_{3}$ at $x_{\mathrm{RW}}\left(\nu_{3}\right)$, such that $\xi_{\mathrm{RW}}(0)=\xi_{0}$. Moreover, the rotating wave $\mathrm{SO}(3) x_{0}$ persists to all nearby momentum values.

b) Assume that $\mathbb{J}_{\mathcal{N}_{1}} \mathrm{D}_{w}^{2} h(0)$ has no eigenvalues in $\mathrm{i} \omega_{0}^{\text {rot }} \mathbb{Z}$. Then there is a two-parameter family $\mathcal{P}_{\mathrm{MRW}}\left(\nu_{2}, \nu_{3}\right), \nu_{2} \geq 0, \nu_{3} \approx 0$, of modulated rotating waves of (3.1) such that $x_{\mathrm{MRW}}\left(\nu_{2}, \nu_{3}\right) \in \mathcal{P}_{\mathrm{MRW}}\left(\nu_{2}, \nu_{3}\right)$ is smooth in $\left(\nu_{2}, \nu_{3}\right)$ and $x_{\mathrm{MRW}}\left(0, \nu_{3}\right)=x_{\mathrm{RW}}\left(\nu_{3}\right)$. The modulated rotating wave at $x_{\mathrm{MRW}}\left(\nu_{2}, \nu_{3}\right)$ has drift symmetry $\gamma_{\mathrm{MRW}}\left(\nu_{2}, \nu_{3}\right)$, relative period $T\left(\nu_{2}, \nu_{3}\right)$ and momentum $\mathbf{J}\left(x_{\mathrm{MRW}}\left(\nu_{2}, \nu_{3}\right)\right)=\left(0, \nu_{2}, \nu_{3}\right)$, and $x_{\mathrm{MRW}}(0,0)=x_{0}$, $T(0,0)=2 \pi /\left|\omega_{0}^{\mathrm{rot}}\right|, \gamma_{\mathrm{MRW}}(0)=\mathrm{id}$. This family contains a one-parameter family $\mathcal{P}\left(\nu_{2}, 0\right)$ of modulated rotating waves which have an average drift velocity $\xi\left(\nu_{2}, 0\right)$ at $x_{\mathrm{MRW}}\left(\nu_{2}, 0\right)$ parallel to the $e_{2}$-axis.

c) Assume that the rotating wave $\mathrm{SO}(3) x_{0}$ is elliptic and nonresonant in the sense of Definition 4.4 and that $\mathbb{J}_{\mathcal{N}_{1}} \mathrm{D}_{w}^{2} h(0)$ has no eigenvalues $\mathrm{i} \omega_{j}$ with $\omega_{0}^{\text {rot }} / \omega_{j} \in \mathbb{Z}$. Then there are $\left(\frac{1}{2} \operatorname{dim} \mathcal{M}-3\right)$ more two-parameter families $\mathcal{P}_{j}\left(\nu_{3}, s\right), j=1, \ldots \frac{1}{2} \operatorname{dim} \mathcal{M}-3$, of modulated rotating waves near the rotating wave and there are smooth functions $x_{j}\left(\nu_{3}, s\right) \in \mathcal{P}_{j}\left(\nu_{3}, s\right)$ with $x_{j}\left(\nu_{3}, 0\right)=x_{\mathrm{RW}}\left(\nu_{3}\right)$ (where $x_{\mathrm{RW}}\left(\nu_{3}\right)$ is from part a)). The modulated rotating wave $\mathcal{P}_{j}\left(\nu_{3}, s\right)$ has momentum $\mathbf{J}\left(x_{j}\left(\nu_{3}, s\right)\right)=\left(0,0, \nu_{3}\right)$ at $x_{j}\left(\nu_{3}, s\right)$, energy $H\left(x_{j}\left(\nu_{3}, s\right)\right)=$ $H\left(x_{\mathrm{RW}}\left(\nu_{3}\right)\right) \pm s^{2}$ (depending on the Krein signature of $\left.\omega_{j}\right)$, relative period $T_{j}\left(\nu_{3}, s\right)$ such that $T_{j}(0,0)=2 \pi /\left|\omega_{j}\right|$, and average drift velocity $\xi_{j}\left(\nu_{3}, s\right) \| e_{3}, s \geq 0$, with $\xi_{j}(0,0)=\xi_{0}$, $j=1, \ldots \frac{1}{2} \operatorname{dim} \mathcal{M}-3$.

Proof. If the rotating wave $\mathrm{SO}(3) x_{0}$ has momentum $\mu_{0}=0$ then $\mathbf{g}_{\mu_{0}}=\mathrm{so}(3)$. In this case $\nu \in \mathrm{so}(3)^{*} \simeq \mathbb{R}^{3}$ and the $\dot{\nu}$-equation from (3.19) becomes

$$
\dot{\nu}=\nu \times \mathrm{D}_{\nu} h(\nu, w) .
$$

a) Since the $\dot{\nu}$ equation has nontrivial dynamics, let us reduce only by the symmetry group

$$
\tilde{\Gamma}=\left\{\gamma \in \mathrm{SO}(3), \quad \operatorname{Ad}_{\gamma} \xi_{0}=\xi_{0}\right\}=\mathrm{SO}_{3}
$$

which is the group of rotations around the $e_{3}$-axis. The corresponding slice is denoted by $\widetilde{\mathcal{N}}=\widetilde{\mathcal{N}}_{0} \oplus \widetilde{\mathcal{N}}_{1}$. Then $\tilde{\nu} \in \widetilde{\mathcal{N}}_{0}$ is given by $\tilde{\nu}=\nu_{3}$ and $\dot{\tilde{\nu}}=0$. Note that $\operatorname{dim} \widetilde{\mathcal{N}}_{1}=$ $\operatorname{dim} \mathcal{N}_{1}+4$. Let $\tilde{h}(\tilde{\nu}, \tilde{w})$ be the Hamiltonian in the bundle coordinates $(\tilde{\gamma}, \tilde{\nu}, \tilde{w}) \in \tilde{\Gamma} \times$ $\widetilde{\mathcal{N}}_{0} \oplus \widetilde{\mathcal{N}}_{1}$. The matrix ad $\xi_{\xi_{0}}^{*}$ has eigenvalues $\pm \mathrm{i} \omega_{0}^{\text {rot }}$ with real eigenspace $\left\{\nu=\left(\nu_{1}, \nu_{2}, 0\right) \in\right.$ 
so $\left.(3)^{*}\right\}$ and a simple eigenvalue 0. Because of (2.4) and (3.20), the eigenvalues of the linearization $\mathbb{J}_{\widetilde{\mathcal{N}}_{1}} \mathrm{D}_{\tilde{w}}^{2} \tilde{h}(0)$ of the equilibrium $0 \in \widetilde{\mathcal{N}}_{1}$ corresponding to the rotating wave $\mathrm{SO}(3) x_{0}$ are given by the eigenvalues of $\mathbb{J}_{\mathcal{N}_{1}} \mathrm{D}_{w}^{2} h(0)$ and by the eigenvalues $\pm \mathrm{i} \omega_{0}^{\text {rot }}$ of multiplicity two. Hence the rotating wave is nondegenerate when considered as a rotating wave of a Hamiltonian system with $\mathrm{SO}_{3}(2)$-symmetry. Therefore $\dot{\tilde{w}}=0$ for $\tilde{w}(\tilde{\nu})$ can be solved using the nondegeneracy condition. This gives rotating waves $\mathrm{SO}(3) x_{\mathrm{RW}}(\tilde{\nu})$, $x_{\mathrm{RW}}(\tilde{\nu}) \simeq(\mathrm{id}, \tilde{\nu}, \tilde{w}(\tilde{\nu}))$, for the original $\mathrm{SO}(3)$-equivariant Hamiltonian system (3.1). Since only a reduction by $\mathrm{SO}_{3}(2)$-symmetry was carried out, these rotating waves have drift velocities $\xi_{\mathrm{RW}}\left(\nu_{3}\right) \| e_{3}$ where $\xi(0)=\xi_{0} \neq 0$. Then (3.16) implies that also $\mathbf{J}\left(x\left(\nu_{3}\right)\right) \| e_{3}$. This proves part a).

b) The rotating waves through $x_{\mathrm{RW}}\left(\nu_{3}\right) \simeq\left(\gamma_{\mathrm{RW}}\left(\nu_{3}\right), \nu_{\mathrm{RW}}\left(\nu_{3}\right), w_{\mathrm{RW}}\left(\nu_{3}\right)\right)$ from part a) have, by (3.18), momentum

$$
\mathbf{J}\left(x_{\mathrm{RW}}\left(\nu_{3}\right)\right)=\left(0,0, \nu_{3}\right)=\gamma_{\mathrm{RW}}\left(\nu_{3}\right) \nu_{\mathrm{RW}}\left(\nu_{3}\right)
$$

and energy $E_{\mathrm{RW}}\left(\nu_{3}\right)=h\left(\nu_{\mathrm{RW}}\left(\nu_{3}\right), w_{\mathrm{RW}}\left(\nu_{3}\right)\right)$ where $E_{\mathrm{RW}}^{\prime}(0)=\mathrm{D}_{\nu} h(0) \nu_{\mathrm{RW}}^{\prime}(0)=\omega_{0}^{\mathrm{rot}} \neq 0$. Therefore they can be parametrized by energy $E$ instead of $\nu_{3}$. Denote the corresponding path of rotating waves again by $x_{\mathrm{RW}}(E) \simeq\left(\gamma_{\mathrm{RW}}(E), \nu_{\mathrm{RW}}(E), w_{\mathrm{RW}}(E)\right)$. Let $\omega^{\text {rot }}(E)$ be the rotation frequency of the rotating wave $\mathrm{SO}(3) x_{\mathrm{RW}}(E)$. Since the slice equation conserves the energy $h(\nu, w)$ the energy level sets $\mathcal{N}_{E}$ of $\mathcal{N}$ are flow-invariant. Because of $\mathrm{D}_{\nu} h(0)=\left(0,0, \omega_{0}^{\text {rot }}\right), \omega_{0}^{\text {rot }} \neq 0$, and $\mathrm{D}_{w} h(0)=0$, they can be parametrized, similarly as in the proof of Theorem 4.3 , by

$\mathcal{N}_{E}=\{(\nu, w) \in \mathcal{N}, \quad h(\nu, w)=E\}=\left\{(\nu, w) \in \mathcal{N}, \nu_{3}=\nu_{3}\left(\nu_{1}, \nu_{2}, w, E\right)\right\}, \quad E \approx E_{0}=h(0)$.

Then $\nu_{3}\left(\nu_{\mathrm{RW}, 1}(E), \nu_{\mathrm{RW}, 2}(E), w_{\mathrm{RW}}(E), E\right)=\nu_{\mathrm{RW}, 3}(E)$. Let us now consider the equilibrium $v_{\mathrm{RW}}(E):=\left(\nu_{\mathrm{RW}}(E), w_{\mathrm{RW}}(E)\right)$ of the slice equation as periodic orbit with period $T^{\mathrm{rot}}(E)=\frac{2 \pi}{\omega^{\mathrm{rot}}(E) \mid}$. The matrix $\operatorname{ad}_{\xi_{0}}^{*}$ has a pair $\pm \mathrm{i} \omega_{0}^{\text {rot }}$ of non-vanishing imaginary eigenvalues with real eigenspace spanned by the vectors $\left\{\left(\nu_{1}, \nu_{2}, 0\right), \nu_{1}, \nu_{2} \in \mathbb{R}\right\} \subseteq \operatorname{so}(3)^{*}$. By (3.20), the linearization of the slice equation $\mathrm{D}_{v} f_{\mathcal{N}}\left(v_{\mathrm{RW}}(E)\right)$ at $E=E_{0}$ also has this pair of eigenvalues which perturbs to the eigenvalues $\pm \mathrm{i} \omega^{\mathrm{rot}}(E)$ of $\mathrm{D}_{v} f_{\mathcal{N}}\left(v_{\mathrm{RW}}(E)\right)$ for $E \approx E_{0}$. Consequently,

$$
\begin{gathered}
\mathcal{S}_{E}=\left\{(\nu, w) \in \mathcal{N}_{E}, \quad \nu_{1}=\nu_{\mathrm{RW}, 1}(E), \nu_{2}>\nu_{\mathrm{RW}, 2}(E), \nu_{2} \approx \nu_{\mathrm{RW}, 2}(E),\right. \\
\left.\nu_{3}=\nu_{3}\left(\nu_{\mathrm{RW}, 1}(E), \nu_{2}, w, E\right), w \approx w_{\mathrm{RW}}(E)\right\}
\end{gathered}
$$

is a section transverse to the flow of the slice equation at $v_{\mathrm{RW}}(E)$ inside the energy level set $\mathcal{N}_{E}$ to the energy $E \approx E_{0}$. Let $s:=\nu_{2}-\nu_{\mathrm{RW}, 2}(E)$. The corresponding Poincaré map is denoted by $\Pi(\cdot, E): \mathcal{S}_{E} \rightarrow \mathcal{S}_{E}$. Decompose $\Pi(\cdot, E)=\left(\Pi_{0}(\cdot, E), \Pi_{1}(\cdot, E)\right)$ where $\Pi_{0}$ maps into the ray $s \geq 0$ and $\Pi_{1}$ into $\mathcal{N}_{1}$. By assumption there is no $k: 1$-resonance between $\omega_{0}^{\text {rot }}$ and any normal frequency on $\mathcal{N}_{1}$. Therefore the equation $\Pi_{1}(s, w, E)=w$ can be solved for $w(s, E)$, such that $w(0, E)=w_{\mathrm{RW}}(E)$ for $E \approx E_{0}$. Plugging this into $\Pi_{0}$ a map $\tilde{\Pi}(\cdot, E)$ from the ray $s \geq 0$ into itself is obtained. The $\dot{\nu}$-equation (5.1) conserves the Casimir $C^{R}(\nu, w)=\|\nu\|_{2}^{2}$. Define $\nu_{3}(s, E):=\nu_{3}\left(\nu_{\mathrm{RW}, 1}(E), s+\nu_{\mathrm{RW}, 2}(E), w(s, E), E\right)$. Then $\hat{s}=\hat{\Pi}(s, E)$ satisfies $C(\hat{s}, E)=C(s, E)$ where

$$
C(s, E)=\left(\nu_{\mathrm{RW}, 1}(E)\right)^{2}+\left(\nu_{\mathrm{RW}, 2}(E)+s\right)^{2}+\left(\nu_{3}(s, E)\right)^{2} .
$$

The path of relative equilibria $\mathrm{SO}(3) x_{\mathrm{RW}}(E)$ corresponds to $(s, E)=(0, E)$. Note that

$$
\mathrm{D} h\left(v_{\mathrm{RW}}(E)\right) \| \mathrm{D} C^{R}\left(v_{\mathrm{RW}}(E)\right)
$$

and that $\left.\mathrm{D} C^{R}\right|_{h(v)=E}=0$ at $v=v_{\mathrm{RW}}(E)$. As a result of this, $\mathrm{D}_{s} C(0, E) \equiv 0$. Moreover, from $\mathrm{D}_{\nu} h(0)=\omega_{0}^{\text {rot }} e_{3}$ and $\mathrm{D}_{w} h(0)=0$, it follows that $\mathrm{D}_{s} \nu_{3}\left(0, E_{0}\right)=0$, and therefore $\mathrm{D}_{s}^{2} C\left(0, E_{0}\right)=2$. Hence $s \rightarrow C(s, E)$ is injective for $s \geq 0, s \approx 0$, for any fixed $E \approx E_{0}$. Consequently $\hat{s}=s$, and so $v(s, E):=(\nu(s, E), w(s, E))$, with $\nu(s, E)=\left(\nu_{\mathrm{RW}, 1}(E), s+\right.$ 
$\left.\nu_{\mathrm{RW}, 2}(E), \nu_{3}(s, E)\right)^{T}$, lies on a periodic orbit of the slice equation with period $T(s, E) \approx$ $T(0, E)=\frac{2 \pi}{\omega^{\text {rot }}(E) \mid}$.

Changing the parametrization back from $E$ to $\nu_{3}$, a two-parameter family $v\left(s, \nu_{3}\right)=$ $\left(\nu\left(s, \nu_{3}\right), w\left(s, \nu_{3}\right)\right)$ of periodic orbits of the slice equation with periods $T\left(s, \nu_{3}\right)$ is obtained, satisfying $T\left(0, \nu_{3}\right)=T^{\mathrm{rot}}\left(0, \nu_{3}\right)$. These give points $\hat{x}\left(s, \nu_{3}\right) \simeq\left(\gamma_{\mathrm{RW}}\left(\nu_{3}\right), v\left(s, \nu_{3}\right)\right)$ on modulated rotating waves of the full Hamiltonian system (3.1) with momentum $\hat{\mu}\left(s, \nu_{3}\right)=\gamma_{\mathrm{RW}}\left(\nu_{3}\right) \nu\left(s, \nu_{3}\right)$, see (3.18). At the rotating waves $\hat{\mu}\left(0, \nu_{3}\right)=\nu_{3}$ and hence $\mathrm{D}_{\nu_{3}} \hat{\mu}\left(0, \nu_{3}\right) \equiv e_{3}$. Moreover $\gamma_{\mathrm{RW}}(0)=\mathrm{id}$ implies that $\mathrm{D}_{s} \hat{\mu}(0,0) \equiv e_{2}$. So smooth functions $\hat{\phi}_{2}\left(s, \nu_{3}\right), \hat{\phi}_{3}\left(s, \nu_{3}\right)$ can be found such that $\hat{\gamma}\left(s, \nu_{3}\right)=\exp \left(\hat{\phi}_{2}\left(s, \nu_{3}\right) \xi_{2}+\hat{\phi}_{3}\left(s, \nu_{3}\right) \xi_{3}\right)$ (with the notation from $(2.9))$ satisfies $\left(\hat{\gamma}\left(s, \nu_{3}\right) \hat{\mu}\left(s, \nu_{3}\right)\right)_{1}=0$ and $\hat{\phi}_{2}\left(0, \nu_{3}\right)=0, \hat{\phi}_{3}\left(0, \nu_{3}\right)=0$. Let $\gamma\left(s, \nu_{3}\right)=\hat{\gamma}\left(s, \nu_{3}\right) \gamma_{\mathrm{RW}}\left(\nu_{3}\right)$. Then $x\left(s, \nu_{3}\right) \simeq\left(\gamma\left(s, \nu_{3}\right), v\left(s, \nu_{3}\right)\right)$ lies on an RPO with momentum $\mu\left(s, \nu_{3}\right)$ such that $\mu_{1}\left(s, \nu_{3}\right)=0$. Then $s$ can be replaced by $\nu_{2}$ and $\left(\nu_{2}, \nu_{3}\right)$ can be tranformed such that $x\left(\nu_{2}, \nu_{3}\right)$ has momentum $\mu\left(\nu_{2}, \nu_{3}\right)=\mathbf{J}\left(x\left(\nu_{2}, \nu_{3}\right)\right)=\left(0, \nu_{2}, \nu_{3}\right)$, $\nu_{2} \geq 0$.

The condition (3.17) implies that the drift symmetry $\gamma\left(\nu_{2}, \nu_{3}\right)$ at the RPO through $x\left(\nu_{2}, \nu_{3}\right)$ satisfies $\gamma\left(\nu_{2}, \nu_{3}\right) \mu\left(\nu_{2}, \nu_{3}\right)=\mu\left(\nu_{2}, \nu_{3}\right)$, where $\gamma(0)=\mathrm{id}$. Therefore $\gamma\left(\nu_{2}, \nu_{3}\right)$ is a rotation around the vector $\mu\left(\nu_{2}, \nu_{3}\right)$ in the $\left(x_{2}, x_{3}\right)$-plane. Moreover for $\nu_{3}=0$ the modulated rotating wave $\mathcal{P}\left(\nu_{2}, 0\right)$ rotates around the $e_{2}$ axis with momentum $\mu\left(\nu_{2}\right)=\left(0, \nu_{2}, 0\right)$ at $x\left(\nu_{2}, 0\right)$.

c) By assumption there is no $k: 1$-resonance between any of the eigenvalues of $\mathbb{J}_{\mathcal{N}_{1}} \mathrm{D}_{w}^{2} h(0)$ and between the eigenvalues of $\mathbb{J}_{\mathcal{N}_{1}} \mathrm{D}_{w}^{2} h(0)$ and $\mathrm{i} \omega_{0}^{\text {rot }}$. Hence for all normal frequencies on $\mathcal{N}_{1}$, part c) follows from the Lyapounov Centre Theorem applied on the space $\tilde{\mathcal{N}}_{1}$, after symmetry reduction by $\mathrm{SO}_{3}(2)$ as in part a).

\subsection{Hamiltonian meandering transition with the Euclidean symmetry of three-dimensional space}

In this section the Hamiltonian analogue of the resonance drift of Remark $2.2 \mathrm{c}$ ) is studied. The symmetry group is again $\Gamma=\mathrm{SE}(3)=\mathrm{SO}(3) \ltimes \mathbb{R}^{3}$. Similarly as in (3.8), (3.9), the adjoint and coadjoint actions for $\Gamma=\mathrm{SE}(3)$ are

$$
\begin{aligned}
& \operatorname{Ad}_{(R, a)} \xi=\left(R \xi^{r}, R \xi^{a}-R \xi^{r} \times a\right) \\
& \operatorname{Ad}_{(R, a)^{-1}}^{*} \mu=\left(R \mu^{r}+a \times R \mu^{a}, R \mu^{a}\right),
\end{aligned}
$$

where $(R, a) \in \mathrm{SO}(3) \ltimes \mathbb{R}^{3}$, see e.g. $[15,26]$. So typically, when $\mu^{a} \neq 0$, then $\Gamma_{\mu} \simeq \operatorname{SO}(2) \times \mathbb{R}$. In this case resonance drift is not possible:

Proposition 5.3 Let SE(3) $x_{0}$ be a nondegenerate relative equilibrium with generic momentum value $\mu_{0}$ satisfying $\mu_{0}^{a} \neq 0$ and with drift velocity $\xi_{0}$. Align $x_{0}$ such that $\xi_{0}^{a}\left\|e_{3}, \xi_{0}^{r}\right\| e_{3}$. Then the following holds true:

a) There is a two-parameter family $\mathrm{SE}(3) x_{\mathrm{RE}}\left(\nu_{3}^{r}, \nu_{3}^{a}\right)$ of relative equilibria of (3.1) with $x_{\mathrm{RE}}(0,0)=x_{0}$. The relative equilibrium at $x_{\mathrm{RE}}\left(\nu_{3}^{r}, \nu_{3}^{a}\right)$ has angular momentum $\mathbf{J}^{r}\left(x_{\mathrm{RE}}\left(\nu_{3}^{r}, \nu_{3}^{a}\right)\right)=\left(0,0, \mu_{0,3}^{r}+\nu_{3}^{r}\right)$, linear momentum $\mathbf{J}^{r}\left(x_{\mathrm{RE}}\left(\nu_{3}^{r}, \nu_{3}^{a}\right)\right)=\left(0,0, \mu_{0,3}^{a}+\nu_{3}^{a}\right)$ and drift velocity $\xi_{\mathrm{RE}}\left(\nu_{3}^{r}, \nu_{3}^{a}\right)$, which satisfies $\xi_{\mathrm{RE}}^{r}\left(\nu_{3}^{r}, \nu_{3}^{a}\right)\left\|e_{3}, \xi_{\mathrm{RE}}^{a}\left(\nu_{3}^{r}, \nu_{3}^{a}\right)\right\| e_{3}$.

b) Let the relative equilibrium $\mathrm{SE}(3) x_{0}$ be elliptic and nonresonant in the sense of Definition 4.4 and denote its normal frequencies by $\omega_{j}, j=1, \ldots, \frac{1}{2} \operatorname{dim} \mathcal{M}-4$. Then there are $\left(\frac{1}{2} \operatorname{dim} \mathcal{M}-4\right)$ families of $R P O s \mathcal{P}_{j}\left(\nu_{3}^{r}, \nu_{3}^{a}, s\right), s \geq 0$, and smooth functions $x_{j}\left(\nu_{3}^{r}, \nu_{3}^{a}, s\right) \in \mathcal{P}_{j}\left(\nu_{3}^{r}, \nu_{3}^{a}, s\right)$ such that $x_{j}\left(\nu_{3}^{r}, \nu_{3}^{a}, 0\right)=x_{\mathrm{RE}}\left(\nu_{3}^{r}, \nu_{3}^{a}\right)$. The RPO at $x_{j}\left(\nu_{3}^{r}, \nu_{3}^{a}, s\right)$ has momentum

$$
\mathbf{J}^{r}\left(x_{j}\left(\nu_{3}^{r}, \nu_{3}^{a}, s\right)\right)=\left(0,0, \mu_{0,3}^{r}+\nu_{3}^{r}\right), \quad \mathbf{J}^{a}\left(x_{j}\left(\nu_{3}^{r}, \nu_{3}^{a}, s\right)\right)=\left(0,0, \mu_{0,3}^{a}+\nu_{3}^{a}\right),
$$


energy $\left.H\left(x_{j}\left(\nu_{3}^{r}, \nu_{3}^{a}, s\right)\right)=H_{\mathrm{RE}}\left(\nu_{3}^{r}, \nu_{3}^{a}\right)\right) \pm s^{2}$ (depending on the Krein signature of $\left.\omega_{j}\right)$, relative period $T_{j}\left(\nu_{3}^{r}, \nu_{3}^{a}, s\right)$, such that $T_{j}(0,0,0)=2 \pi /\left|\omega_{j}\right|$, and average drift velocity $\xi_{j}\left(\nu_{3}^{r}, \nu_{3}^{a}, s\right)$ at $x_{j}\left(\nu_{3}^{r}, \nu_{3}^{a}, s\right)$, which satisfies $\xi_{j}^{r}\left(\nu_{3}^{r}, \nu_{3}^{a}, s\right)\left\|e_{3}, \xi_{j}^{a}\left(\nu_{3}^{r}, \nu_{3}^{a}, s\right)\right\| e_{3}, \xi_{j}(0,0)=\xi_{0}$.

\section{Proof.}

a) Note that, by (5.2), $\xi_{0}^{a}\left\|e_{3}, \xi_{0}^{r}\right\| e_{3}$ implies $\mu_{0}^{a}\left\|e_{3}, \mu_{0}^{r}\right\| e_{3}$. The Lie-group $\Gamma_{\mu_{0}} \simeq \mathrm{SO}_{3}(2) \times \mathbb{R}_{3}$ is abelian, therefore $\dot{\nu} \equiv 0$ holds in the equations (3.19) near a relative equilibrium $\mathrm{SE}(3) x_{0}$ with generic momentum value. By the nondegeneracy condition, $\dot{w}=0$ can be solved for $w_{\mathrm{RE}}(\nu)$ to obtain relative equilibria through $x_{\mathrm{RE}}(\nu) \simeq\left(\mathrm{id}, \nu, w_{\mathrm{RE}}(\nu)\right)$. The statement about the momentum of $x_{\mathrm{RE}}(\nu)$ follows from (3.18), the statement about the velocity $\xi_{\mathrm{RE}}(\nu)$ from (3.16) and (5.2).

b) The Lyapounov Centre Theorem can be applied on the $\nu$-dependent $\dot{w}$-equation. The statements about the momentum of the families of RPOs follows from (3.18). The fact that their drift symmetry lies in $\mathrm{SO}_{3}(2) \times \mathbb{R}_{3}$, and hence their average drift velocity in $\mathrm{so}_{3}(2) \times \mathbb{R}_{3}$, follow from (3.17) and the fact that $\Gamma_{\mu_{0}} \simeq \mathrm{SO}_{3}(2) \times \mathbb{R}_{3}$, see part a).

The situation is different if the relative equilibrium $\mathrm{SE}(3) x_{0}$ has a nongeneric momentum value $\mu_{0}=\mathbf{J}\left(x_{0}\right)$. In what follows, it is shown that in this case resonance drift occurs generically.

If $\mu_{0}^{a}=0$ then (5.2) implies that $\Gamma_{\mu_{0}} \simeq \mathrm{SO}(2) \ltimes \mathbb{R}^{3}$. Let us assume, without loss of generality, that $\mu_{0}^{r} \| e_{3}$. Then the drift velocity $\xi_{0}=\left(\xi_{0}^{r}, \xi_{0}^{a}\right)$ of the relative equilibrium at $x_{0}$ satisfies $\xi_{0}^{r} \| e_{3}$. Choose $x_{0}$ in its $\mathrm{SE}(3)$ orbit such that also $\xi_{0}^{a} \| e_{3}$. The momentum value $\mu_{0}$ is non-split, and the $\dot{\nu}$-equation of (3.19) for $\nu=\left(\nu^{r}, \nu^{a}\right) \in \operatorname{so}(2)^{*} \oplus\left(\mathbb{R}^{3}\right)^{*}$ is non-trivial, see [26]. It can be easily checked that the functions

$$
C^{a}(\mu)=\left\|\mu^{a}\right\|^{2} \quad \text { and } \quad C^{r}(\mu)=\left\langle\mu^{a}, \mu^{r}\right\rangle
$$

are invariant under the coadjoint action (5.2). These restrict to the functions $C^{a}(\nu)=\left\|\nu^{a}\right\|^{2}$ and $C^{r}(\nu)=\nu_{3}^{a}\left(\mu_{3}^{r}+\nu^{r}\right)$ on the slice $\mathcal{N}_{0} \simeq \operatorname{so}(2)^{*} \oplus\left(\mathbb{R}^{3}\right)^{*}$ and are Casimirs, i.e., conserved quantities of the $\dot{\nu}$-equation.

In the following proposition persistence of a relative equilibrium with vanishing linear momentum is studied, as a prerequisite for the analysis of the Hamiltonian meandering transition.

Proposition 5.4 Let $\mathrm{SE}(3) x_{0}$ be a nondegenerate relative equilibrium with momentum value $\mu_{0}=\left(\mu_{0}^{r}, 0\right), \mu_{0}^{r} \| e_{3}$, and with drift velocity $\xi_{0}=\left(\xi_{0}^{r}, \xi_{0}^{a}\right)$, where $\xi_{0}^{r} \neq 0, \xi_{0}^{r}\left\|e_{3}, \xi_{0}^{a}\right\| e_{3}$. Then there exists a 2-dimensional family of relative equilibria $\mathrm{SE}(3) x_{\mathrm{RE}}\left(\nu^{r}, \nu_{3}^{a}\right)$ of (3.1) such that $x_{\mathrm{RE}}\left(\nu^{r}, \nu_{3}^{a}\right)$ is smooth in its parameters and $x_{\mathrm{RE}}(0)=x_{0}, \xi_{\mathrm{RE}}(0,0)=\xi_{0}$. The relative equilibrium through $x_{\mathrm{RE}}\left(\nu^{r}, \nu_{3}^{a}\right)$ has angular momentum $\mathbf{J}^{r}\left(x_{\mathrm{RE}}\left(\nu^{r}, \nu_{3}^{a}\right)\right)=\mu_{0}^{r}+\nu^{r} e_{3}$, linear momentum $\mathbf{J}^{a}\left(x_{\mathrm{RE}}\left(\nu^{r}, \nu_{3}^{a}\right)\right)=\nu_{3}^{a} e_{3}$ and drift velocity $\xi_{\mathrm{RE}}\left(\nu^{r}, \nu_{3}^{a}\right)$, where $\xi_{\mathrm{RE}}^{r}\left(\nu^{r}, \nu_{3}^{a}\right)=\omega^{\mathrm{rot}}\left(\nu^{r}, \nu_{3}^{a}\right) e_{3}$, $\xi_{\mathrm{RE}}^{a}\left(\nu^{r}, \nu_{3}^{a}\right) \| e_{3}$.

Proof. This proposition is an application of a persistence result for general noncompact symmetry groups, see [32, Example 5.3a)]. But it can also be proved in an elementary way: Because of (2.4), (3.20), the linearization $L_{0}$ at $x_{0}$ has, by our nondegeneracy condition and the form of $\operatorname{ad}_{\xi_{0}}$ from (5.2), a 4-dimensional kernel corresponding to two zero eigenvalues of $\operatorname{ad}_{\xi_{0}}$. Therefore let us reduce only by the abelian symmetry group $\tilde{\Gamma}=\mathrm{SO}_{3}(2) \times \mathbb{R}_{3}$ of rotations around and translations along the $e_{3}$-axis. This gives the reduced system

$$
\dot{\tilde{\nu}}=0, \quad \dot{\tilde{w}}=\mathbb{J}_{\widetilde{\mathcal{N}}_{1}} \mathrm{D}_{\tilde{w}} \tilde{h}(\tilde{\nu}, \tilde{w})
$$

on the slice $\tilde{\mathcal{N}}=\widetilde{\mathcal{N}}_{0} \oplus \widetilde{\mathcal{N}}_{1}$, where $\tilde{\nu}=\left(\nu^{r}, \nu_{3}^{a}\right) \in \widetilde{\mathcal{N}}_{0} \simeq \operatorname{so}_{3}(2)^{*} \oplus \mathbb{R}_{3}^{*}$. Since $\mathbb{J}_{\widetilde{\mathcal{N}}_{1}} \mathrm{D}_{\tilde{w}}^{2} \tilde{h}(0)$ is invertible, the equation $0=\mathbb{J}_{\widetilde{\mathcal{N}}_{1}} \mathrm{D}_{\tilde{w}} h(\tilde{\nu}, \tilde{w})$ can be solved for $\tilde{w}(\tilde{\nu})$ by the implicit function theorem. This gives relative equilibria $\mathrm{SE}(3) x_{\mathrm{RE}}\left(\nu^{r}, \nu_{3}^{a}\right)$ of $(3.1)$, where $x_{\mathrm{RE}}\left(\nu^{r}, \nu_{3}^{a}\right) \simeq(\mathrm{id}, \tilde{\nu}, \tilde{w}(\tilde{\nu}))$. The drift velocity $\xi_{\mathrm{RE}}\left(\nu^{r}, \nu_{3}^{a}\right)$ of the relative equilibrium at $x_{\mathrm{RE}}\left(\nu^{r}, \nu_{3}^{a}\right)$ lies in the Lie algebra of $\tilde{\Gamma}$ and therefore satisfies $\xi_{\mathrm{RE}}^{r}\left(\nu^{r}, \nu_{3}^{a}\right)=\omega_{\mathrm{RE}}^{\mathrm{rot}}\left(\nu^{r}, \nu_{3}^{a}\right) e_{3}$ and $\xi_{\mathrm{RE}}^{a}\left(\nu^{r}, \nu_{3}^{a}\right) \| e_{3}$. The statement about the momentum of $x_{\mathrm{RE}}\left(\nu^{r}, \nu_{3}^{a}\right)$ follows from (3.18). 
In this case resonance drift occurs as the following theorem shows:

Theorem 5.5 Let, as before, $\mathrm{SE}(3) x_{0}$ be a nondegenerate relative equilibrium with momentum $\mu_{0}=\left(\mu_{0}^{r}, 0\right), \mu_{0}^{r} \| e_{3}, \mu_{0}^{r} \neq 0$, and with non-vanishing rotational velocity vector $\xi_{0}^{r} \neq 0$ such that $\xi_{0}^{r}=\omega_{0}^{\text {rot }} e_{3}, \xi_{0}^{a} \| e_{3}$. Assume that $\mathbb{J}_{\mathcal{N}_{1}} \mathrm{D}_{w}^{2} h(0)$ has no eigenvalue in $\mathrm{i} \omega_{0}^{\text {rot }} \mathbb{Z}$. Then:

a) there is a 3-dimensional manifold $\mathcal{P}\left(\nu^{r}, \nu_{2}^{a}, \nu_{3}^{a}\right)$ of RPOs closeby such that $x\left(\nu^{r}, \nu_{2}^{a}, \nu_{3}^{a}\right) \in$ $\mathcal{P}\left(\nu^{r}, \nu_{2}^{a}, \nu_{3}^{a}\right)$ is a smooth function of its parameters, $\nu_{2}^{a} \geq 0, \nu^{r} \approx 0, \nu_{3}^{a} \approx 0$, and $x\left(\nu^{r}, 0, \nu_{3}^{a}\right)=x_{\mathrm{RE}}\left(\nu^{r}, \nu_{3}^{a}\right)$. The RPO through $x\left(\nu^{r}, \nu_{2}^{a}, \nu_{3}^{a}\right)$ has angular momentum $\mathbf{J}^{r}\left(x\left(\nu^{r}, \nu_{2}^{a}, \nu_{3}^{a}\right)\right)=\mu_{0}^{r}+\nu^{r} e_{3}$, linear momentum $\mathbf{J}^{a}\left(x\left(\nu^{r}, \nu_{2}^{a}, \nu_{3}^{a}\right)\right)=\left(0, \nu_{2}^{a}, \nu_{3}^{a}\right)$, and relative period $T\left(\nu^{r}, \nu_{2}^{a}, \nu_{3}^{a}\right)$ with $T\left(\nu^{r}, 0, \nu_{3}^{a}\right)=2 \pi /\left|\omega^{\mathrm{rot}}\left(\nu^{r}, \nu_{3}^{a}\right)\right|$. Here $\omega^{\mathrm{rot}}\left(\nu^{r}, \nu_{3}^{a}\right)$ is the rotation frequency of the relative equilibrium $\mathrm{SE}(3) x_{\mathrm{RE}}\left(\nu^{r}, \nu_{3}^{a}\right)$ from Proposition 5.4.

b) This family contains a 2-dimensional submanifold $x\left(\nu^{r}, \nu_{2}^{a}, 0\right)$ at $\nu_{3}^{a}=0$ which has an average rotational drift velocity $\xi^{r}\left(\nu^{r}, \nu_{2}^{a}, 0\right)$ with $\xi_{3}^{r}\left(\nu^{r}, \nu_{2}^{a}, 0\right)=0$.

Note that the RPO through $x\left(\nu^{r}, \nu_{2}^{a}, 0\right)$ rotates around and translates along a vector parallel to $e_{2}$ whereas the original relative equilibrium through $x_{0}$ rotates around and translates along the $e_{3}$ direction.

Proof of Theorem 5.5. Let $\nu^{r} \approx 0, \nu_{3}^{a} \approx 0$. Near the relative equilibria through $x_{\mathrm{RE}}\left(\nu^{r}, \nu_{3}^{a}\right) \simeq$ $\left(\gamma_{\mathrm{RE}}\left(\nu^{r}, \nu_{3}^{a}\right), \nu_{\mathrm{RE}}\left(\nu^{r}, \nu_{3}^{a}\right), w_{\mathrm{RE}}\left(\nu^{r}, \nu_{3}^{a}\right)\right)$ from Proposition 5.4, let us change coordinates on the slice $\mathcal{N}=\mathcal{N}_{0} \oplus \mathcal{N}_{1}, \mathcal{N}_{0} \simeq \operatorname{so}(2)^{*} \oplus\left(\mathbb{R}^{3}\right)^{*}$, from $v=\left(\nu^{r}, \nu_{1}^{a}, \nu_{2}^{a}, \nu_{3}^{a}, w\right)$ to $\left(E, C^{r}, \nu_{1}^{a}, \nu_{2}^{a}, w\right)$ as follows: first let

$$
\nu_{3}^{a}\left(\nu^{r}, C^{r}\right)=\frac{C^{r}}{\nu^{r}+\mu_{0,3}^{r}} .
$$

Here $C^{r}(\mu+\nu)=\left\langle\nu^{a}, \mu_{0}^{r}+\nu^{r} e_{3}\right)$ is the Casimir from (5.3) restricted to elements of the form $\mu_{0}+\nu$, where $\nu \in \mathcal{N}_{0} \simeq \mathrm{so}_{3}(2)^{*} \oplus\left(\mathbb{R}^{3}\right)^{*}$. Then, by the implicit function theorem, using that $\mathrm{D}_{\nu^{r}} h(0)=\omega_{0}^{\text {rot }} \neq 0, \mathrm{D}_{w} h(0)=0, \mathrm{D}_{\nu^{a}} h(0) \| e_{3}$ and $\mathrm{D}_{\nu^{r}} \nu_{3}^{a}(0,0)=0$, one obtains

$$
\nu^{r}=\nu^{r}\left(E, C^{r}, \nu_{1}^{a}, \nu_{2}^{a}, w\right)
$$

for $E \approx E_{0}=H\left(x_{0}\right), C^{r} \approx 0$, where $E=h\left(\left(\nu^{r}, \nu_{1}^{a}, \nu_{2}^{a}, \nu_{3}^{a}\left(\nu^{r}, C^{r}\right)\right), w\right)$. Solving $E=$ $h\left(\nu_{\mathrm{RE}}\left(\nu^{r}, \nu_{3}^{a}\left(\nu^{r}, C^{r}\right)\right), w_{\mathrm{RE}}\left(\nu^{r}, \nu_{3}^{a}\left(\nu^{r}, C^{r}\right)\right)\right)$ by the implicit function theorem for $\nu^{r}$, the family of relative equilibria $\operatorname{SE}(3) x_{\mathrm{RE}}\left(E, C^{r}\right), x_{\mathrm{RE}}\left(E, C^{r}\right) \simeq\left(\gamma_{\mathrm{RE}}\left(E, C^{r}\right), v_{\mathrm{RE}}\left(E, C^{r}\right)\right), v_{\mathrm{RE}}\left(E, C^{r}\right)=$ $\left(\nu_{\mathrm{RE}}\left(E, C^{r}\right), w_{\mathrm{RE}}\left(E, C^{r}\right)\right)$, is obtained, parametrized by the conserved quantities $\left(E, C^{r}\right)$. Then, since $\omega_{0}^{\text {rot }} \neq 0$ and $\xi_{0}^{r}=\omega_{0}^{\text {rot }} e_{3}$, by (3.20) and (5.2), for $C^{r} \approx 0, E \approx E_{0}$, the section

$$
\begin{gathered}
\mathcal{S}_{E, C^{r}}=\left\{(\nu, w) \in \mathcal{N}, \nu_{3}^{a}=\nu_{3}^{a}\left(\nu^{r}, C^{r}\right), \nu^{r}=\nu^{r}\left(E, C^{r}, \nu_{1}^{a}, \nu_{2}^{a}, w\right), \nu_{1}^{a}=\nu_{\mathrm{RE}, 1}^{a}\left(E, C^{r}\right),\right. \\
\left.\nu_{2}^{a}>\nu_{\mathrm{RE}, 2}^{a}\left(E, C^{r}\right), \nu_{2}^{a} \approx \nu_{\mathrm{RE}, 2}^{a}\left(E, C^{r}\right), w \approx w_{\mathrm{RE}}\left(E, C^{r}\right)\right\}
\end{gathered}
$$

is transversal to the flow of (3.1) in the flow-invariant manifold

$$
\mathcal{N}_{E, C^{r}}:=\left\{(\nu, w) \in \mathcal{N}, h(\nu, w)=E, C^{r}(\nu)=C^{r}\right\} .
$$

Consider the Poincaré map $(s, w) \rightarrow \Pi\left(E, C^{r}, s, w\right)$ from $\mathcal{S}_{E, C^{r}}$ to itself where $s=\nu_{2}^{a}-$ $\nu_{\mathrm{RE}, 2}\left(E, C^{r}\right)$. At $\left(E, C^{r}, s, w\right)=\left(E_{0}, 0,0,0\right)$ the Poincaré return time is $T(0)=\frac{2 \pi}{\left|\omega_{0}^{\text {rot }}\right|}$. Due to the nonresonance assumption the equation $\Pi_{1}\left(E, C^{r}, s, w\right)=w$ can be solved for $w\left(E, C^{r}, s\right)$ such that $w\left(E, C^{r}, 0\right)=w_{\mathrm{RE}}\left(E, C^{r}\right)$. Here $\Pi_{1}$ is the $\mathcal{N}_{1}$ component of $\Pi$. What follows, is a proof that $\hat{x}\left(E, C^{r}, s\right)=\left(\gamma_{\mathrm{RE}}\left(E, C^{r}\right), \nu\left(E, C^{r}, s\right), w\left(E, C^{r}, s\right)\right)$ lies on an RPO. Here

$$
\begin{aligned}
\nu\left(E, C^{r}, s\right) & =\left(\nu^{r}\left(E, C^{r}, s\right), \nu^{a}\left(E, C^{r}, s\right)\right), \\
\nu^{r}\left(E, C^{r}, s\right) & =\nu^{r}\left(E, C^{r}, \nu_{\mathrm{RE}, 1}^{a}\left(E, C^{r}\right), s+\nu_{\mathrm{RE}, 2}^{a}\left(E, C^{r}\right), w\left(E, C^{r}, s\right)\right), \\
\nu_{3}^{a}\left(E, C^{r}, s\right) & =\nu_{3}^{a}\left(\nu^{r}\left(E, C^{r}, s\right), C^{r}\right), \\
\nu^{a}\left(E, C^{r}, s\right) & =\left(\nu_{\mathrm{RE}, 1}^{a}\left(E, C^{r}\right), s+\nu_{\mathrm{RE}, 2}^{a}\left(E, C^{r}\right), \nu_{3}^{a}\left(E, C^{r}, s\right)\right) .
\end{aligned}
$$


By construction, $\hat{x}\left(E, C^{r}, 0\right)=x_{\mathrm{RE}}\left(E, C^{r}\right)$. Define

$$
C^{a}\left(E, C^{r}, s\right)=\left\|\nu^{a}\left(E, C^{r}, s\right)\right\|^{2} .
$$

The equilibria $v_{\mathrm{RE}}\left(E, C^{r}\right)$ of the slice equation are at $s=0$. The fact that $\mathrm{D}_{(\nu, w)} h$ is a linear combination of $\mathrm{D}_{(\nu, w)} C^{a}$ and $\mathrm{D}_{(\nu, w)} C^{r}$ at any equilibrium of the slice equation then implies that

$$
\mathrm{D}_{s} C^{a}\left(E, C^{r}, 0\right)=0 .
$$

Moreover $\mathrm{D}_{s} \nu_{3}^{a}(0,0,0)=0$ since $\nu_{3}^{a}\left(\nu^{r}, C^{r}=0\right) \equiv 0$. This together with (5.5) gives

$$
\mathrm{D}_{s}^{2} C^{a}\left(E_{0}, 0,0\right)=2 .
$$

So for small positive $s$ and fixed $E \approx E_{0}, C^{r} \approx 0$, the function $s \rightarrow C^{a}\left(E, C^{r}, s\right)$ is injective. This proves that there is a coordinate transformation from $\left(E, C^{r}, s\right)$ to the conserved quantities $\left(E, C^{r}, C^{a}\right)$. Therefore $\hat{x}\left(E, C^{r}, s\right)$ lies on a relative periodic orbit $\mathcal{P}\left(E, C^{r}, s\right)$ of $(3.1)$, as claimed. Let us now change coordinates back from $\left(E, C^{r}, s\right)$ to $\left(\nu^{r}, s, \nu_{3}^{a}\right)$ and denote the corresponding function again by $\hat{x}\left(\nu^{r}, s, \nu_{3}^{a}\right) \simeq\left(\gamma_{\mathrm{RE}}\left(\nu^{r}, \nu_{3}^{a}\right), \nu\left(\nu^{r}, s, \nu_{3}^{a}\right), w\left(\nu^{r}, s, \nu_{3}^{a}\right)\right) \in \mathcal{P}\left(\nu^{r}, s, \nu_{3}^{a}\right)$.

It is now shown that a smooth function $\hat{\gamma}\left(\nu^{r}, s, \nu_{3}^{a}\right)$ can be found such that

$$
x\left(\nu^{r}, s, \nu_{3}^{a}\right) \simeq\left(\gamma\left(\nu^{r}, s, \nu_{3}^{a}\right), v\left(\nu^{r}, s, \nu_{3}^{a}\right)\right),
$$

where $\gamma\left(\nu^{r}, s, \nu_{3}^{a}\right)=\hat{\gamma}\left(\nu^{r}, s, \nu_{3}^{a}\right) \gamma_{\mathrm{RE}}\left(\nu^{r}, \nu_{3}^{a}\right)$ and $v\left(\nu^{r}, s, \nu_{3}^{a}\right)=\left(\nu\left(\nu^{r}, s, \nu_{3}^{a}\right), w\left(\nu^{r}, s, \nu_{3}^{a}\right)\right)$, satisfies

$$
\mathbf{J}_{1}^{a}\left(x\left(\nu^{r}, s, \nu_{3}^{a}\right)\right)=0, \quad \mathbf{J}_{1}^{r}\left(x\left(\nu^{r}, s, \nu_{3}^{a}\right)\right)=0, \quad \mathbf{J}_{2}^{r}\left(x\left(\nu^{r}, s, \nu_{3}^{a}\right)\right)=0,
$$

and $\hat{\gamma}\left(\nu^{r}, 0, \nu_{3}^{a}\right)=$ id. First note that this holds true at $s=0$ by Proposition 5.4. For $s \neq 0$, let

$$
\hat{\gamma}\left(\nu^{r}, s, \nu_{3}^{a}\right)=\left(\hat{R}\left(\nu^{r}, s, \nu_{3}^{a}\right), \hat{a}\left(\nu^{r}, s, \nu_{3}^{a}\right)\right),
$$

and $\hat{\mu}\left(\nu^{r}, s, \nu_{3}^{a}\right)=\mathbf{J}\left(\hat{x}\left(\nu^{r}, s, \nu_{3}^{a}\right)\right)$. Then $\mathbf{J}^{a}\left(x_{\mathrm{RE}}\left(\nu^{r}, \nu_{3}^{a}\right)\right)=\nu_{3}^{a} e_{3}$ implies $\mathrm{D}_{\nu_{3}^{a}} \hat{\mu}^{a}(0,0,0)=e_{3}$. From $\gamma_{\mathrm{RE}}(0,0)=\mathrm{id}$ one further gets $\mathrm{D}_{s} \hat{\mu}^{a}(0,0,0)=e_{2}$. Therefore smooth functions $\hat{\phi}_{2}\left(\nu^{r}, s, \nu_{3}^{a}\right), \hat{\phi}_{3}\left(\nu^{r}, s, \nu_{3}^{a}\right)$ can be found such that $\hat{\phi}_{j}\left(\nu^{r}, 0, \nu_{3}^{a}\right)=0, j=2,3$, and

$$
\left(\hat{R}\left(\nu^{r}, s, \nu_{3}^{a}\right) \hat{\mu}^{a}\left(\nu^{r}, s, \nu_{3}^{a}\right)\right)_{1}=0
$$

where, as in (2.9),

$$
\hat{R}\left(\nu^{r}, s, \nu_{3}^{a}\right)=\exp \left(\hat{\phi}_{2}\left(\nu^{r}, s, \nu_{3}^{a}\right) \xi_{2}+\hat{\phi}_{3}\left(\nu^{r}, s, \nu_{3}^{a}\right) \xi_{3}\right) .
$$

In this way the first equation of (5.6) is satisfied. Then $\nu_{3}^{a}$ and $\nu_{2}^{a}=s$ can be rescaled such that

$$
\mathbf{J}^{a}\left(x\left(\nu^{r}, \nu_{2}^{a}, \nu_{3}^{a}\right)\right)=\left(0, \nu_{2}^{a}, \nu_{3}^{a}\right)
$$

Let

$$
\tilde{\mu}\left(\nu^{r}, \nu_{2}^{a}, \nu_{3}^{a}\right)=\operatorname{Ad}_{\left(\hat{R}\left(\nu^{r}, \nu_{2}^{a}, \nu_{3}^{a}\right), 0\right)^{-1}}^{*} \hat{\mu}\left(\nu^{r}, \nu_{2}^{a}, \nu_{3}^{a}\right) .
$$

Note that $\tilde{\mu}^{a}\left(\nu^{r}, \nu_{2}^{a}, \nu_{3}^{a}\right)=\left(0, \nu_{2}^{a}, \nu_{3}^{a}\right)^{T}$ has been achieved by choosing $\hat{R}\left(\nu^{r}, \nu_{2}^{a}, \nu_{3}^{a}\right)$. Moreover (5.2) gives

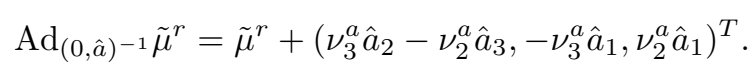

Let $\hat{a}_{2} \equiv 0$. When $\nu_{2}^{a} \neq 0$, then $\hat{a}_{3}=\hat{a}_{3}\left(\nu^{r}, \nu_{2}^{a}, \nu_{3}^{a}\right)$ can be chosen such that

$$
\left(\operatorname{Ad}_{\left(0, \hat{a}\left(\nu^{r}, \nu_{2}^{a}, \nu_{3}^{a}\right)\right)^{-1}}^{*} \tilde{\mu}^{r}\left(\nu^{r}, \nu_{2}^{a}, \nu_{3}^{a}\right)\right)_{1}=0
$$

thus satisfying the second equation of (5.6). If $\nu_{2}^{a}=0$, i.e. at the relative equilibria, $\tilde{\mu}^{r}\left(\nu^{r}, 0, \nu_{3}^{a}\right)=\nu^{r} e_{3}$ anyway and when $\nu_{2}^{a} \rightarrow 0$ then $\hat{a}_{3} \rightarrow \mathrm{D}_{\nu_{2}^{a}} \tilde{\mu}_{1}^{r}\left(\nu^{r}, 0, \nu_{3}^{a}\right)$. Moreover the equation

$$
\left(\operatorname{Ad}_{\left(0, \hat{a}\left(\nu^{r}, \nu_{2}^{a}, \nu_{3}^{a}\right)\right)^{-1}}^{*} \tilde{\mu}^{r}\left(\nu^{r}, \nu_{2}^{a}, \nu_{3}^{a}\right)\right)_{2}=0
$$


and hence the last equation of (5.6), can be satisfied by choosing $\hat{a}_{1}\left(\nu^{r}, \nu_{2}^{a}, \nu_{3}^{a}\right)$ appropriately whenever $\nu_{3}^{a} \neq 0$. When $\nu_{3}^{a}=0$ then $C^{r}=0$ and $\nu_{\mathrm{RE}}\left(\nu^{r}, 0\right)=\left(\nu^{r}, 0\right)$. In particular $\nu_{\mathrm{RE}, 1}^{a}\left(\nu^{r}, 0\right)=0$, and so also $\nu_{1}^{a}\left(\nu^{r}, s, 0\right)=0$ (see $\left.(5.5)\right)$. Therefore $\hat{R}\left(\nu^{r}, s, 0\right)=\mathrm{id}$ and $\tilde{\mu}_{2}^{r}\left(\nu^{r}, \nu_{2}^{a}, 0\right)=0$. Consequently, when $\nu_{2}^{a} \rightarrow 0$ then $\hat{a}_{2} \rightarrow \mathrm{D}_{\nu_{2}^{a}} \tilde{\mu}_{2}^{r}\left(\nu^{r}, \nu_{2}^{a}, 0\right)$. Hence a smooth function $\hat{a}\left(\nu^{r}, \nu_{2}^{a}, \nu_{3}^{a}\right)$ has been found such that $\mu^{r}\left(\nu^{r}, \nu_{2}^{a}, \nu_{3}^{a}\right):=\operatorname{Ad}_{(0, \hat{a})^{-1}}^{*} \tilde{\mu}^{r}\left(\nu^{r}, \nu_{2}^{a}, \nu_{3}^{a}\right) \| e_{3}$. For $\nu_{2}^{a}=0$ the equality $\mu^{r}\left(\nu^{r}, 0, \nu_{3}^{a}\right)=\mu_{0}^{r}+\nu^{r} e_{3}$ holds. So coordinates can be changed such that $\mu^{r}\left(\nu^{r}, \nu_{2}^{a}, \nu_{3}^{a}\right)=\mu_{0}^{r}+\nu^{r} e_{3}$ for all $\nu_{2}^{a} \geq 0, \nu_{2}^{a} \approx 0$. This proves part a) of the proposition.

For part b), let $\gamma\left(\nu^{r}, \nu_{2}^{a}, \nu_{3}^{a}\right)=\left(R\left(\nu^{r}, \nu_{2}^{a}, \nu_{3}^{a}\right), a\left(\nu^{r}, \nu_{2}^{a}, \nu_{3}^{a}\right)\right)$ be the drift symmetry of the RPO $\mathcal{P}\left(\nu^{r}, \nu_{2}^{a}, \nu_{3}^{a}\right)$ at $x\left(\nu^{r}, \nu_{2}^{a}, \nu_{3}^{a}\right)$ and write, as in $(2.9), R\left(\nu^{r}, \nu_{2}^{a}, \nu_{3}^{a}\right)=\exp \left(\sum_{i=1}^{3} \phi_{i} \xi_{i}\right)$ where $\phi_{i}=\phi_{i}\left(\nu^{r}, \nu_{2}^{a}, \nu_{3}^{a}\right)$. Then $\phi_{3}\left(\nu^{r}, \nu_{2}^{a}, \nu_{3}^{a}\right)=0$ at $\nu_{3}^{a}=0$ needs to be satisfied. Equations (3.17) and (5.2) imply that $R\left(\nu^{r}, \nu_{2}^{a}, \nu_{3}^{a}\right) \mathbf{J}^{a}\left(x\left(\nu^{r}, \nu_{2}^{a}, \nu_{3}^{a}\right)\right)=\mathbf{J}^{a}\left(x\left(\nu^{r}, \nu_{2}^{a}, \nu_{3}^{a}\right)\right)$ where $\mathbf{J}^{a}\left(x\left(\nu^{r}, \nu_{2}^{a}, \nu_{3}^{a}\right)\right)=$ $\left(0, \nu_{2}^{a}, \nu_{3}^{a}\right)$. Hence $\sum_{i=1}^{3} \phi_{i} \xi_{i}=\hat{\phi}\left(0, \nu_{2}^{a}, \nu_{3}^{a}\right)^{T}$ for some $\hat{\phi} \in \mathbb{R}$, where $\xi_{i}$ is identified with $e_{i} \in \mathbb{R}^{3}$ and so(3) and $\left(\mathbb{R}^{3}\right)^{*}$ with $\mathbb{R}^{3}$. Because of this, $\phi_{3}=0$ for $\nu_{3}^{a}=0$.

In addition to the family of RPOs from the above theorem, there may be additional families of RPOs which rotate and translate about the same axis (without loss of generality the $e_{3}$-axis) as the relative equilibrium:

Proposition 5.6 Let, as before, $\mathrm{SE}(3) x_{0}$ be a relative equilibrium of an $\mathrm{SE}(3)$-equivariant Hamiltonian system (3.1), with momentum $\mu_{0}=\left(\mu_{0}^{r}, 0\right), \mu_{0}^{r} \neq 0$, and with non-vanishing rotational velocity vector $\xi_{0}^{r} \neq 0$. Choose $x_{0}$ such that $\mu_{0}^{r}\left\|e_{3}, \xi_{0}^{r}=\omega_{0}^{\text {rot }} e_{3}, \xi_{0}^{a}\right\| e_{3}$. Assume that the relative equilibium is nonresonant and elliptic in the sense of Definition 4.4 , and that $\omega_{0}^{\mathrm{rot}} / \omega_{j} \notin \mathbb{Z}$ for all eigenvalues $\mathrm{i} \omega_{j}$ of $\mathbb{J}_{\mathcal{N}_{1}} \mathrm{D}_{w}^{2} h(0)$. Then there are 3-dimensional manifolds $\mathcal{P}_{j}\left(\nu^{r}, s, \nu_{3}^{a}\right)$ of RPOs, $j=1, \ldots \frac{1}{2} \operatorname{dim} \mathcal{M}-5$, and smooth functions $x_{j}\left(\nu^{r}, s, \nu_{3}^{a}\right) \in \mathcal{P}_{j}\left(\nu^{r}, s, \nu_{3}^{a}\right)$ such that $x_{j}\left(\nu^{r}, 0, \nu_{3}^{a}\right)=x_{\mathrm{RE}}\left(\nu^{r}, \nu_{3}^{a}\right)$ (with $x_{\mathrm{RE}}\left(\nu^{r}, \nu_{3}^{a}\right)$ from Proposition 5.4). Moreover, the RPO through $x_{j}\left(\nu^{r}, s, \nu_{3}^{a}\right)$ has momentum $\left(\mu_{0}^{r}+\nu^{r} e_{3}, \nu_{3}^{a} e_{3}\right)$, energy $H\left(x_{j}\left(\nu^{r}, s, \nu_{3}^{a}\right)\right)=H\left(x_{\mathrm{RE}}\left(\nu^{r}, \nu_{3}^{a}\right)\right) \pm s^{2}(d e-$ pending on the Krein signature of $\left.\omega_{j}\right)$, relative period $T_{j}\left(\nu^{r}, s, \nu_{3}^{a}\right)$, where $T_{j}(0,0,0)=2 \pi /\left|\omega_{j}\right|$, and average drift velocity $\xi_{j}\left(\nu^{r}, s, \nu_{3}^{a}\right)=\left(\xi_{j, 3}^{r}\left(\nu^{r}, s, \nu_{3}^{a}\right) e_{3}, \xi_{j, 3}^{a}\left(\nu^{r}, s, \nu_{3}^{a}\right) e_{3}\right), \xi_{j}(0,0,0)=\xi_{0}$.

Proof. Let us, as in the proof of Proposition 5.4, only reduce by the symmetry group $\tilde{\Gamma}=$ $\mathrm{SO}_{3}(2) \times \mathbb{R}_{3}$. The statement then follows by applying the Lyapounov Centre Theorem on the $\dot{\tilde{w}}$-equation of (5.4).

\section{Conclusion and future directions}

In this paper a Hamiltonian analogue of the well-known meandering transition from rotating waves to modulated rotating and modulated travelling waves in systems with Euclidean symmetries has been studied. This transition occurs for example in a finite-dimensional system of point vortices. Similar effects have been analyzed in systems with spherical symmetry and with the Euclidean symmetry of three-dimensional space. It remains a challenging open problem to extend these results to infinite-dimensional Hamiltonian systems such as PDE models of vortex dynamics.

\section{Acknowledgments}

This work was partially supported by a grant of the Nuffield organization, by EPSRC grant EP/D063906/1 and by a Leverhulme Research Fellowship.

\section{References}

[1] H. Aref. Three-vortex motion with zero total circulation: Addendum. J. Appl. Math. Phys. (ZAMP) 40, 495-500, 1989.

[2] V.I. Arnold and B.A. Khesin. Topological Methods in Hydrodynamics. Springer, New York, Berlin, Heidelberg, 1998. 
[3] D. Barkley. Euclidean symmetry and the dynamics of rotating spiral waves. Phys. Rev. Lett., 72:164-167, 1994. http://link.aps.org/abstract/PRL/v72/p164

[4] D. Chan. Hopf bifurcations from relative equilibria in spherical geometry. J. Differential Equations, 226, 118-134, 2006.

[5] D. Chan. A Normal Form Approach to Nonresonant and Resonant Hopf Bifurcation from Relative Equilibria. PhD Thesis, University of Surrey, 2007.

[6] D. Chan and I. Melbourne. A geometric characterisation of resonance in Hopf bifurcation from relative equilibria. Physica D 234, 98-104, 2007.

[7] A. Comanici. Transition from rotating to modulated rotating waves on the sphere. SIAM J. Appl. Dyn. Syst. 5(4), 759-782, 2006

[8] B. Fiedler, B. Sandstede, A. Scheel, and C. Wulff. Bifurcation from relative equilibria to noncompact group actions: Skew products, meanders, and drifts. Doc. Math. J. DMV, 1:479-505, 1996.

[9] V. Ginzburg and E. Lerman. Existence of relative periodic orbits near relative equilibria. Math. Research Letters 11, 397-412, 2004.

[10] M. Golubitsky, V. LeBlanc, and I. Melbourne. Meandering of the spiral tip - an alternative approach. J. Nonl. Sc., 7:557-586, 1997.

[11] V. Guillemin and S. Sternberg. A normal form for the moment map. In Differential geometric methods in mathematical physics, (S.Sternberg, editor), D.Reidel, Dordrecht, 1984.

[12] V. Guillemin and S. Sternberg. Symplectic techniques in physics. Cambridge University Press, Cambridge, 1984.

[13] G.R. Kirchhoff. Vorlesungen über Mathematische Physik. Mechanik. Teubner, Leipzig, 1876.

[14] M. Krupa. Bifurcations of relative equilibria. SIAM J. Math. Anal., 21:1453-1486, 1990.

[15] N.E. Leonard and J.E. Marsden. Stability and drift of underwater vehicle dynamics: mechanical systems with rigid motion symmetry. Physica D, 105:130-162, 1997.

[16] C.-M. Marle. Modèle d'action Hamiltonienne d'un groupe de Lie sur une variété symplectique. Rendiconti del Seminario Matematico, Università e Politechnico, Torino, 43:227-251, 1985.

[17] J.E. Marsden and T.S. Ratiu. Introduction to Mechanics and Symmetry. Springer-Verlag, New York, Berlin, Heidelberg, 1994.

[18] K.R. Meyer and G.R. Hall. Introduction to Hamiltonian dynamical systems and the N-body problem. Springer-Verlag, New York, 1992.

[19] C. Lim, J.A. Montaldi and R.M. Roberts. Relative equilibria of point vortices on the sphere. Physica D, 148:97-135, 2001.

[20] J. Montaldi and T. Tokieda. Openness of momentum maps and persistence of extremal relative equilibria. Topology 42, 833-844, 2003.

[21] P. Newton. The N-Vortex Problem. Volume 145 of Appl. Math. Sc. Springer-Verlag, 2001.

[22] J.-P. Ortega. Relative normal modes for nonlinear Hamiltonian systems. Proc. Royal Soc. Edinb., Sect. A, Math. 133(3), 675-704, 2003. 
[23] J.-P. Ortega and T. Ratiu. Relative equilibria near stable and unstable Hamiltonian relative equilibria. Proc. R. Soc. Lond. Ser. A Math. Phys. Eng. Sci. 460(2045), 1407-1431, 2004.

[24] R.S. Palais. On the existence of slices for actions of noncompact Lie groups. Ann. of Math., 73:295-323, 1961.

[25] G.W. Patrick. Dynamics of perturbed relative equilibria of point vortices on the sphere or plane. J. Nonlin. Sci. 10, 401-415, 2000.

[26] R.M. Roberts, C. Wulff, and J. Lamb. Hamiltonian systems near relative equilibria. J. Differential Equations, 179, 562-604, 2002.

[27] B. Sandstede, A. Scheel, and C. Wulff. Bifurcations and dynamics of spiral waves. J. Nonlin. Sci., 9(4):439-478, 1999.

[28] U. Scheerer, C. Wulff. Reduced dynamics for momentum maps with cocycle. C. R. Acad. Sc., Série I, Math., 333, 99-104, 2001.

[29] J. L. Synge. On the motion of three vortices. Canadian J. Math., 1, 257-270, 1949.

[30] A.T. Winfree. Varieties of spiral wave behaviour: an experimentalist's approach to the theory of excitable media. Chaos, 1:303-334, 1991.

[31] C. Wulff. Transition from relative equilibria to relative periodic orbits. Doc. Math. J. DMV $5,227-274,2000$.

[32] C. Wulff. Persistence of relative equilibria in Hamiltonian systems with noncompact symmetry. Nonlinearity 16(1), 67-91, 2003.

[33] C. Wulff. Persistence of Hamiltonian relative periodic orbits. J. Geom. Phys., 48, 309-338, 2003.

[34] C. Wulff, J.S.W. Lamb, and I. Melbourne. Bifurcations from relative periodic solutions. Ergodic Theory Dynam. Systems, 21:605-635, 2001.

[35] C. Wulff and M. Roberts. Hamiltonian systems near relative periodic orbits. SIAM J. Appl. Dyn. Syst.,1:1-43, 2002. 